\title{
High Statistics Analysis of All-Neutral Decays of Vector Mesons with the Radphi Experiment
}

Fridah Mokaya

University of Connecticut - Storrs, fridah.mokaya@uconn.edu

\section{Recommended Citation}

Mokaya, Fridah, "High Statistics Analysis of All-Neutral Decays of Vector Mesons with the Radphi Experiment" (2018). Doctoral Dissertations. 1821.

https://opencommons.uconn.edu/dissertations/1821 


\title{
High Statistics Analysis of All-Neutral Decays of Vector Mesons with the Radphi Experiment
}

Fridah Mogoi Mokaya, Ph.D.

University of Connecticut, 2018

\begin{abstract}
The differential cross section and spin density matrix elements for $\omega(782)$ meson photoproduction in the reaction ${ }^{9} \mathrm{Be}(\gamma p, p \omega)^{8} \mathrm{Li}$ have been measured in the energy range 4.4 - $5.4 \mathrm{GeV}$ using the Radphi detector. Radphi used a ${ }^{9}$ Be target, and identified the final state by triggering on the recoil proton plus neutrals. At the kinematics of this experiment, the observed nuclear cross section is consistent with the p cross section measured by prior experiments when multiplied by 4 for the number of protons in the nucleus, in agreement with a naive spectator model. However, the spin observables show some deviation from a spectator model, particularly approaching the low-t limit of the experimental acceptance. Besides the cross sections, spin density matrix elements in Gottfried Jackson and Helicity frame have been measured. The data selection is discussed and the acceptance is examined and quantified.
\end{abstract}




\title{
High Statistics Analysis of All-Neutral Decays of Vector Mesons with the Radphi Experiment
}

\author{
Fridah Mogoi Mokaya
}

M.Sc. Physics, The State University of New York at Binghamton, Binghamton, NY, 2010

\author{
A Dissertation \\ Submitted in Partial Fulfillment of the \\ Requirements for the Degree of \\ Doctor of Philosophy \\ at the \\ University of Connecticut
}

2018 
Copyright by

Fridah Mogoi Mokaya

2018 


\title{
APPROVAL PAGE
}

Doctor of Philosophy Dissertation

\section{High Statistics Analysis of All-Neutral Decays of Vector Mesons with the Radphi Experiment}

\author{
Presented by \\ Fridah Mogoi Mokaya, M.Sc. Phys. \\ Major Advisor \\ Dr Richard Jones \\ Associate Advisor \\ Dr Jeffrey Schweitzer \\ Associate Advisor \\ Dr Peter Schweitzer \\ University of Connecticut \\ 2018
}


To my daughter, Jenise Odongo, reach for the stars. Nothing is impossible if you put your heart into it. . 


\section{ACKNOWLEDGEMENTS}

Firstly, I would like to express my sincere gratitude to my advisor Dr Richard Jones for the continuous support of my Phd study and related research, for his patience, motivation, and immense knowledge. His guidance helped me in all the time of research. Through him I have come to learn a lot.

My thesis committee guided me throughout the years. Thank you to Dr . Peter Schweitzer who always had his door open and answered any questions I had. Dr Jeffrey Schweitzer, thank you very much for all the life lessons you gave me you literally taught me not to be a quitter and through you I learned that any challenge I encounter is a problem waiting to be solved.

Thanks to the members of my committee for their work reading this thesis and also to the Physics department at the University of Connecticut for creating a great atmosphere for learning and growth. I thank my fellow labmates in for the stimulating discussions and their willingness to help always, Alex, Brendan and James thank you.

Nobody has been more important to me in my entire graduate school than my family members. I would like to thank the members of my family and whose love and guidance are with me in whatever I pursue. Most importantly I wish to thank my dad, for believing in me and constant encouragement and spiritual support throughout my life in general.

Finally to my loving husband and daughter, Joshua and Jenise, thank you for being patient and for providing unending inspiration and support. Thank you! 


\section{TABLE OF CONTENTS}

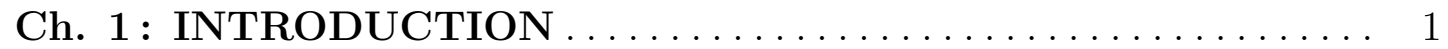

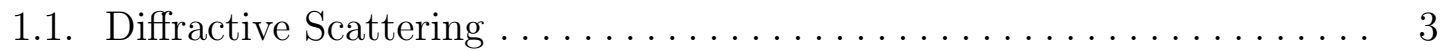

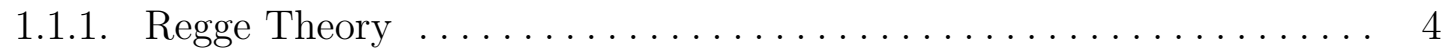

1.2. Radiative Decays of Vector Mesons $\ldots \ldots \ldots \ldots \ldots \ldots \ldots \ldots \ldots$

1.3. Vector Meson Photoproduction $\ldots \ldots \ldots \ldots \ldots \ldots \ldots \ldots \ldots \ldots \ldots$

1.4. Observables in Vector Meson Photoproduction. . . . . . . . . . . . . . . 10

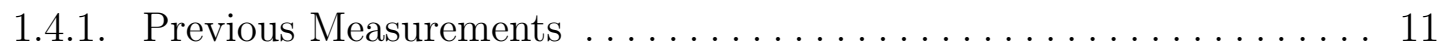

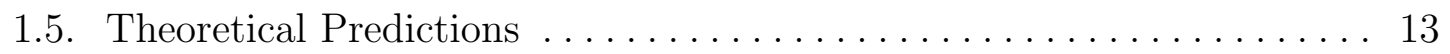

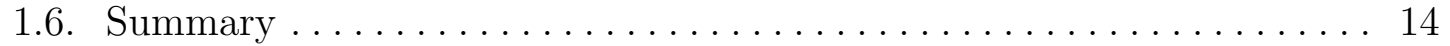

Ch. 2: Spin density matrix elements in $\omega$ photoproduction $\ldots \ldots 16$

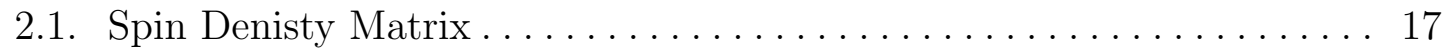

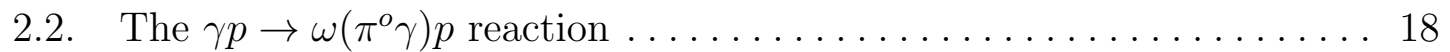

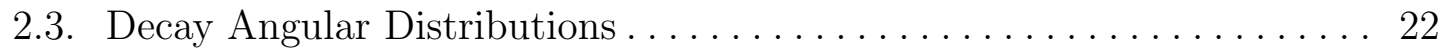

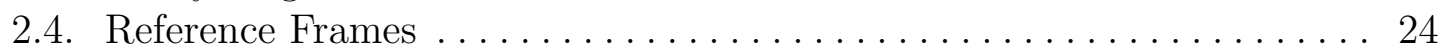

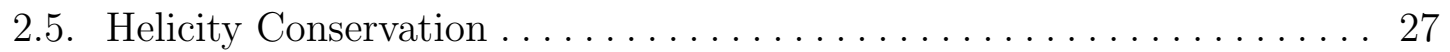

2.5.1. s-channel helicity conservation $\ldots \ldots \ldots \ldots \ldots \ldots \ldots \ldots \ldots \ldots \ldots$

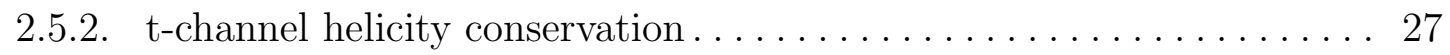

Ch. 3: Experimental Apparatus ..................... 29

3.1. CEBAF Continuous Electron Beam Accelerator Facility . . . . . . . . . . . 29

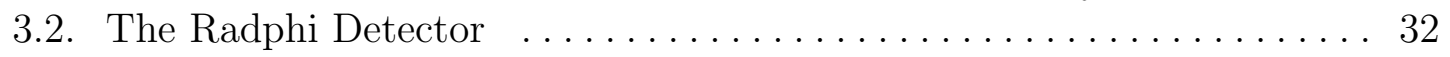

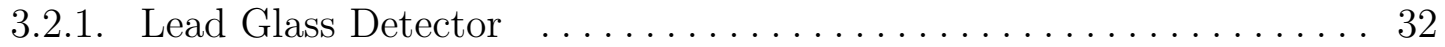

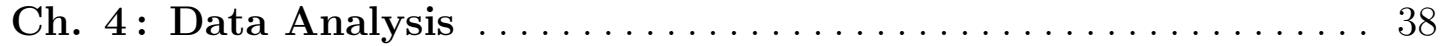

4.1 Event Reconstruction . . . . . . . . . . . . . . . . . . . . . . . . 38

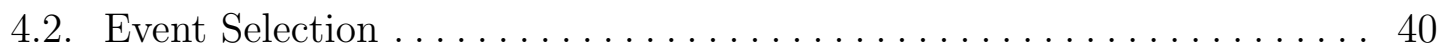

4.2 .1 Selection of $\omega$ events . . . . . . . . . . . . . . . . . . . . . . . . . . 41

4.3 Sideband subtraction . . . . . . . . . . . . . . . . . . . . . 48

4.3 .1 Nucleon Resonances . . . . . . . . . . . . . . . . . . 51

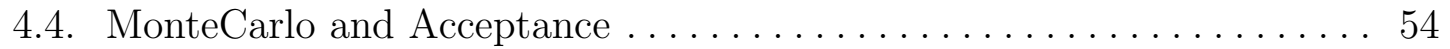

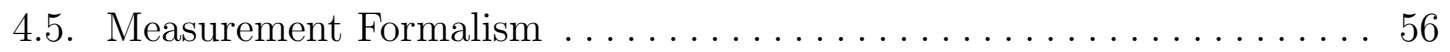

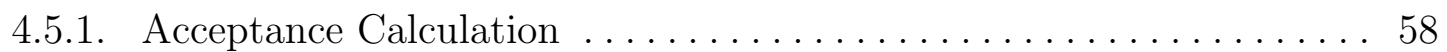

4.5 .2 . Normalization . . . . . . . . . . . . . . . . . . . . . . . . . . . . . . . . . 59

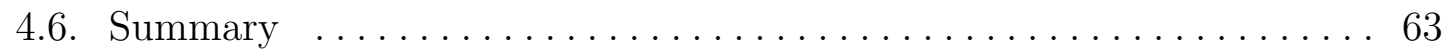

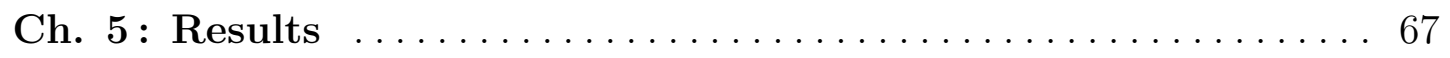

5.1. Spin Density Matrix Elements Extraction $\ldots \ldots \ldots \ldots \ldots \ldots \ldots$. . . . . 67

5.1.1. Maximum Likelihood Fitting $\ldots \ldots \ldots \ldots \ldots \ldots \ldots \ldots \ldots$ 


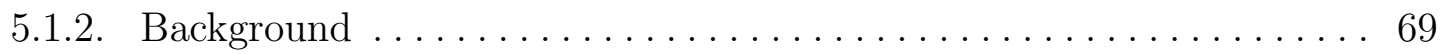

5.1.3. Handling Non-Uniform Weights $\ldots \ldots \ldots \ldots \ldots \ldots \ldots \ldots \ldots \ldots$

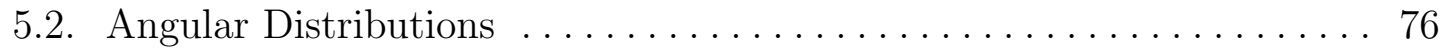

$5.3 . \quad$ Measurement Uncertainity $\ldots \ldots \ldots \ldots \ldots \ldots \ldots \ldots \ldots \ldots \ldots \ldots \ldots$

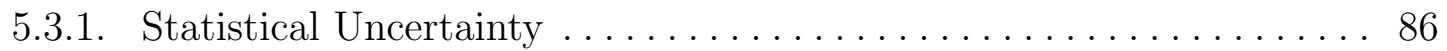

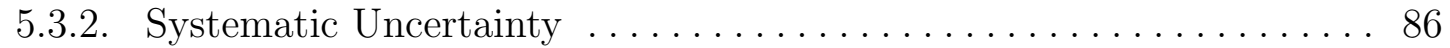

5.4 Cross Section . . . . . . . . . . . . . . . . . . . . . . . . . . . . . 89

5.4.1. Total and differential cross section . . . . . . . . . . . . . . . 90

5.5. Unpolarized Spin Density Matrix Elements $\ldots \ldots \ldots \ldots \ldots \ldots . . \ldots 9$

5.5.1. Comparison with earlier results $\ldots \ldots \ldots \ldots \ldots \ldots \ldots \ldots \ldots$

5.6 . Theoretical Model for Vector Meson Photoproduction ............. 99

5.6.1. The Joint Physics Analysis Center (JPAC) Model ................ 99

Ch. 6: Conclusions . ............................... 103

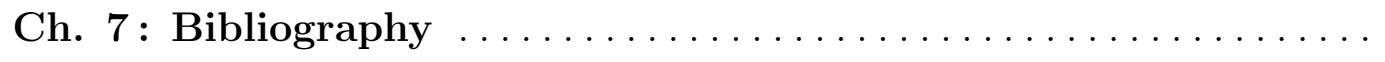




\section{LIST OF FIGURES}

1.3.1 Representation of the VMD for the vector meson photoproduction mechanism with t-channel exchanges. The $\rho, \omega, \phi$ are vector mesons

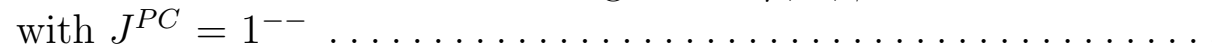

1.4.1 Differential cross section measured by SLAC, the solid circles are the differential cross sections while the dashed lines show the naturall parity-exchange contribution to the differential cross-section. . . . . . 12

1.4 .2 Spin denisty matrix elements and parity assymetry elements, in the helicity frame vs $\mathrm{t} \mid$ from SLAC 4 . . . . . . . . . . . . . 15

2.4.1 Diagrammatic representation of the helicty frame in the $\omega$ frame. . . 25

2.4 .2 Diagrammatic representation of the Gottfried Jackson frame in the

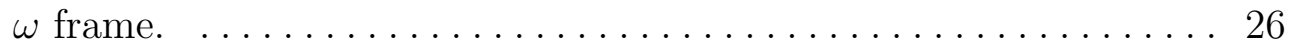

3.1 .1 Schematic diagram of CEBAF during the 6 GeV era . . . . . . . . . 30

3.1.2 Scematic diagram of the tagger spectrometer. Dashed lines represent the trajectories taken by the recoil electron after being deflected by the dipole magnet. . . . . . . . . . . . . . . . . . . 31

3.2.1 A cut-away view of the half of the Radphi detector. The beam enters from the lower-left passing through a hole in the lead shielding wall (foreground) and strikes the beryllium target (small disk at the center of the figure) which is suspended by thin wires from an aluminum ring. Surrounding the target are the barrel scintillator detector (BSD) and barrel gamma detector(BGD). In the forward region is the lead glass detector (LGD) covering polar angles $3^{0} 28^{\circ}$. Immediately in front of the LGD was a scintillator plane (notshown in the figure) which functioned as a charged particle veto (CPV). The scintillator array in the foreground is the upstream particle veto (UPV). Approximately $40 \mathrm{~m}$ upstream of the target was the photon tagger (not shown), where photons with energy between 4.4 and $5.4 \mathrm{GeV}$ were tagged $\ldots \ldots \ldots \ldots \ldots \ldots \ldots \ldots \ldots \ldots \ldots$

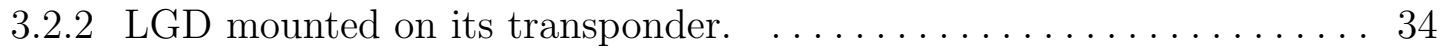

3.2 .3 A schematic view of the Radphi 3-level trigger logic $\ldots \ldots \ldots \ldots \ldots 37$

$4.2 .13 \gamma$ polar angle measured in the lab trame versus total shower energy spectrum distribution (left panel).The distribution on the right

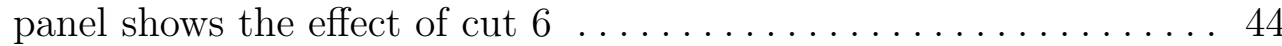


4.2.2 The time difference between a pixel and a hit in the CPV (left) and the tagger (right). Dashed lines in the left panel show the time window used for the charged particle veto. The right panel shows the tagger-pixel time difference before (solid) and after (dashed) the CP veto is applied. The shaded areas in the right panel show the time windows used for counting tagger coincidences (peak region) and accidentals (right of peak region). . . . . . . . . 46

4.2 .3 The mean energy obtained from fitting the tagged total shower energy distribution for each tagging counter plotted as function of mid

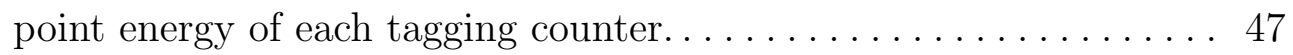

4.2 .4 The invariant mass distribution from the $3 \gamma$ sample obtained after cuts 1-4 above (left panel solid curve), after cuts 1-5 (left panel dashed curve), after cuts 1-6 (right panel solid curve) and after cuts 1-7 (right panel dashed curve). The peak at $0.8 \mathrm{GeV} / \mathrm{c}^{2}$ (right panel) associated with $\omega \rightarrow \pi^{0} \gamma$ events stands out clearly above

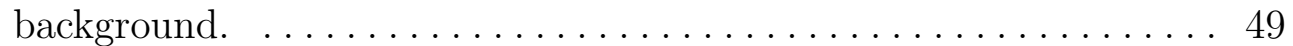

4.2 .5 The invariant mass distribution of all photon pairs from the $3 \gamma \mathrm{sam}-$ ple (solid line). The dashed line corresponds to the lightest pair. The vertical dotted bars show the kinematic selection of the $\pi^{0}$. . 50

4.2.6 The $\pi^{0} \gamma$ invariant mass distribution for the final $3 \gamma$ sample after applying cuts 1-8 and applying the tagger accidental subtraction. The blue curve represents the signal while the green curve repre-

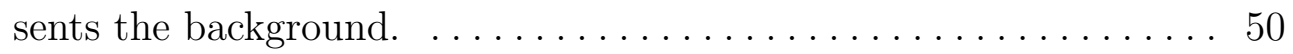

$4.3 .13 \gamma$ invariant mass showing sideband(green) and signal(red) regions. . . 52 4.3 .2 Left panel shows the $\Delta \phi$ distributions from different MC reconstructed reactions while the right panel shows the distribution trom the real data $3 \gamma$ sample. The distribution on the right is fitted to a sum of the four MC distributions shown on the left. The tit is represented by the green curve while the resonance contribution is represented by the blue curve. . . . . . . . . . . . . . . . . . 54

\begin{tabular}{|ll|ll|}
\hline 4.3 .3 & Left panel shows the & $\mathrm{t}$ & distribution from $3 \gamma$ sample before sideband \\
\hline
\end{tabular} subtraction. The distribution on the right is obtained after the sideband subtraction method has been applied. . . . . . . . . . 55

4.5.1 Integrated luminosity. The integrated luminosity for each tagger channel plotted as a function of incident energy. This factor was used in $\gamma p \rightarrow \omega p$ analysis. The y-axis is in units of $\mu^{-1} \mathrm{~b} \ldots \ldots .58$

4.5 .2 Real data out-of-plane momentum distribution for the $3 \gamma$ sample. The $\mathrm{MC}$ distribution is plotted as a red curve $\ldots \ldots \ldots \ldots 61$

4.5.3 Azimuth distribution of the transverse momentum of the forward 37 system after sideband subtraction in invariant mass and $\delta \phi$ from

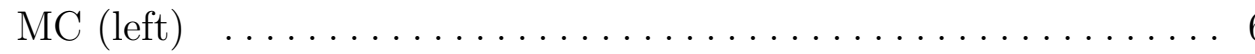


4.5.4 The t-distribution from the $3 \gamma \omega$ sample (left). sideband subtraction in invariant mass has been used to form this plot. MC-acceptance (right) obtained from the ratio of the reconstructed and generated -t distributions with a slope parameter, b, of $5.5 \mathrm{GeV}^{2} \ldots . . .664$

4.5.5 The mean and RMS of the reconstructed $t$ as function of generated $t$ obtained with the MC simulation. The left plot shows the centroid and width in the form of error bars, while the right plot shows the \begin{tabular}{|ll|l|l|}
\hline width separately. Results reported here extend up to & $\mathrm{t}$ & $=1.2$ \\
\hline
\end{tabular}

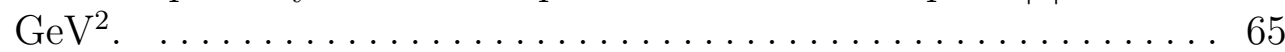

$5.1 .13 \gamma$ invariant mass distributions for selected t-bins. Although the \begin{tabular}{|l|l|l|l|}
\hline background grows as & $\mathrm{t}$ & increases, the lineshape does not vary with \\
\hline
\end{tabular}

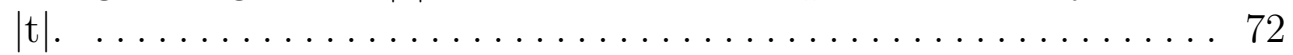

$5.1 .2 \Delta \phi$ distribution for selected t-bins. These distributions show changing $\Delta \phi=0$ peak width as a function of $t \mid \ldots \ldots \ldots \ldots \ldots \ldots$

5.1 .3 R-function used for maximum likelihood fitting for weighted events for a selected t-bin. To form this plot, events with acceptance below $30 \%$ have been eliminated $\ldots \ldots \ldots \ldots \ldots \ldots \ldots \ldots$

5.1.4 Fit function Equation 2.3.4 returned by the fit plotted as a 2D histogram (left) panel for a selected t-bin. This plot is obtained after fitting an angular distribution that has not been etched The right

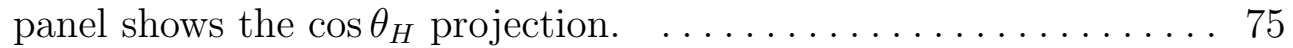

5.1 .5 Fit function Equation 2.3.4 returned by the fit over the data that has been etched to remove regions where there is no experimental data (left panel). The right panel shows the $\cos \theta_{H}$ projection. . . . 76

5.2 .1 Acceptance-corrected helicity frame $\cos \theta_{H}$ distributions for selected t-bins, obtained by taking the ratio of the experimentally measured $\cos \theta_{H}$ and Monte Carlo reconstructed $\cos \theta_{H}$ distributions. The scale of the $y$ axis is arbitrary. These plots show how the $\cos \theta_{H}$ shape varies over the full range in t for the experiment. The errors displayed are statistical. . . . . . . . . . . . . . . . . . 77

5.2 .2 Acceptance-corrected helicity frame $\phi_{H}$ distribution for selected tbins, obtained by taking the ratio of the experimentally measured $\phi_{H}$ and Monte Carlo reconstructed $\phi_{H}$ distributions. The scale of the y axis is arbitrary. These plots show how the $\phi_{H}$ shape varies over the full range in t for the experiment. The errors displayed are statistical . . . . . . . . . . . . . . . . . . . . . . . 78

5.2 .3 Acceptance-corrected Gottfried Jackson frame $\cos \theta_{G J}$ distribution for selected $\mathrm{t}$ | bins, obtained by taking the ratio of the experimentally measured $\cos \theta_{G J}$ and Monte Carlo reconstructed $\cos \theta_{G .}$. distributions. The scale of the y axis is arbitrary. These plots show how the $\cos \theta_{G . J}$ shape varies over the full range in t for the experiment. The errors displayed are statistical . . . . . . . . . . 79 
5.2.4 Acceptance-corrected Gottfried Jackson frame $\phi_{G J}$ distribution for \begin{tabular}{l|l|l|l|} 
selected & $\mathrm{t}$ & bins, obtained by taking ratio of the experimentally \\
\hline
\end{tabular} measured $\phi_{G . J}$ and Monte Carlo reconstructed $\phi_{G . J}$ distributions. These plots show how the $\phi_{G . J}$ shape varies over the full range in t for the experiment. The errors displayed are statistical . . . . . . . 8 80

5.2 .5 Experimental data $\phi_{H}$ versus $\cos \theta_{H}$ from the $\omega$ decay in t-bins for the enrgy range (4.4-5.4) GeV. Each $2 \mathrm{D}$ distribution is labelled by the t-bin in which it was obtained . . . . . . . . . . . . . 82

5.2 .6 Helicity frame Monte Carlo $\phi_{H}$ versus $\cos \theta_{H}$ weighted by the results of the fit model. The agreement between Figure 5.2.5 and weighted Monte Carlo is good . . . . . . . . . . . . . . . . . . 83

5.2.7 Experimental data $\phi_{G J}$ versus $\cos \theta_{G J}$ from the $\omega$ decay in t-bins for the enrgy range (4.4-5.4) GeV. Each 2D distribution is labelled by the $t$-bin in which it was obtained. . . . . . . . . . . . . . . . . . 84

5.2 .8 gottfried Jackson frame Monte Carlo $\phi_{G . J}$ versus $\cos \theta_{G . J}$ weighted by the result of the the fit model. The agreement between Figure 5.2.7 and weighted Monte Carlo is good $\ldots \ldots \ldots \ldots \ldots \ldots . \ldots \ldots$

5.4.1 The total $\omega$ photoproduction cross section normalized per proton in the ${ }^{9}$ Be target, cross section as a function of tagged photon energy(black dots, leftpanel). Measurements from previous experiments are shown for comparison. The right panel shows the slope of the -t distributions as a function of tagged photon energy. . . . . . 90

\begin{tabular}{|lll|l|l|}
\hline 5.4 .2 & Differential cross section for $\gamma p \rightarrow \omega p$ as a function of & $\mathrm{t}$ & . To form \\
\hline
\end{tabular} this plot data from all tagged photon energies shown in Figure 5.4.1 have been summed. Vertical error bars represent statistical error while the horizontal error bars reflect the bin size.The systematic error is shown as the grey shaded area at the bottom of the plot. . 92

5.5.1 Spin density matrix elements for the reaction $\gamma p \rightarrow \omega p$ on a nuclear target in the helicity frame, as a function of momentum transfer $\mathrm{t} \mid$. The vertical error bars indicated are statistical. SDMEs published by previous experiments are represented by open squares SLAC 44 and open circles LAMP2 collaboration 5 . . . . . . . . . . . . . . 94

5.5.2 Gottfried Jackson spin density matrix elements for the reaction $\gamma p \rightarrow$ \begin{tabular}{|l|l|l|}
$\omega p$ on a nuclear target, as a function of & $\mathrm{t}$ & in the energy range 4.39 \\
\hline
\end{tabular} $5.39 \mathrm{GeV}$. The vertical error bars are statistical. Results published by the LAMP2 collaboration are represented by open circles 5 . . . 95

5.5.3 SDME component $\rho_{00}^{0}$ for different incident photon energies and selected t-bin ranges in the Helicity frame. . . . . . . . . . . . . 97

5.5.4 SDME component $\operatorname{Re} \rho_{10}^{0}$ for different incident photon energies and selected t-bin ranges in the Helicity trame. . . . . . . . . . . . . 97

5.5 .5 SDME component $\rho_{1-1}^{0}$ for different incident photon energies and selected t-bin ranges in the Helicity frame. $\ldots \ldots \ldots \ldots \ldots$. . . . 97 
5.5.6 Incident energy dependance of $\rho_{00}^{0}$ for selected t-bins in the Gottfried

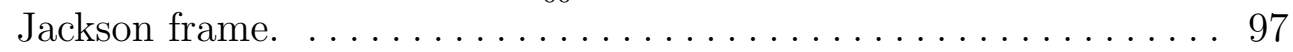

5.5.7 Incident energy dependance of the $\operatorname{Re} \rho_{10}^{0}$ for selected t-bins in the Gottfried Jackson trame . . . . . . . . . . . . . . . . . . 98

5.5.8 Incident beam dependanceof the $\rho_{1-1}^{0}$ component for selected t-bins in the Gottfried lackson frame $\ldots \ldots \ldots \ldots \ldots \ldots \ldots \ldots, 98 \ldots \ldots \ldots$

5.6.1 Schematic representation of t-channel meson and Pomeron exchanges

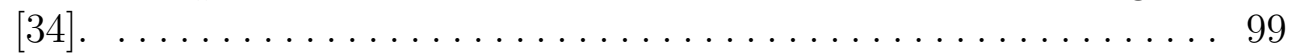

5.6 .2 Helicity frame comparison of the Radphi experiment SDMEs to the

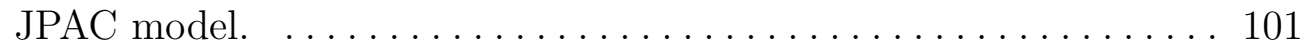

5.6.3 The JPAC model compared to our measurements in the Gottfried

Dackson frame ................................... 102 


\section{LIST OF TABLES}

1.0 .1 Quantum numbers for the two lightest $q \bar{q}$ nonets. . . . . . . . . . . 3

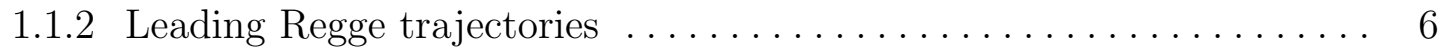

4.2 .3 Table showing $\pi^{0}$ and $\omega$ decay modes and branching fractions . . . . . . 40

4.2 .4 Results of fitting the $3 \gamma$ invariant mass distributions from the sample

with 3 reconstructed showers. The reported errors are statistical

only. The width of $53 \mathrm{MeV} / \mathrm{c}^{2}$ is dominated by the mass resolution

of the detector. The centroid is shifted away from the physical mass

at $0.782 \mathrm{GeV} / \mathrm{c}^{2}$ by approximately half this width due to a slight

systematic bias in the energy calibration of the LGD based on the

$\pi^{0}$ peak. No attempt was made to artificially correct for this bias.

4.5.5 The widths $\left(\sigma_{1}\right.$ and $\left.\sigma_{2}\right)$ and relative heights $\left(f_{1}\right.$ and $\left.f_{2}\right)$ of the two

Gaussians that describe the out-of-plane recoil proton momentum

distribution seen in the real data, compared with that seen in re-

constructed MC. In the MC events the out-of-plane recoil pro-

ton momentum distribution is controlled by the initial state nu-

clear momentum distribution described by a sum of two Gaussians

parametrized as shown in the last row. The parameters of that dis-

tribution were adjusted to obtain a good agreement between the

reconstructed $\mathrm{MC}$ and the real data. Given errors are statisticall

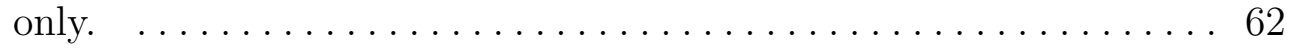

4.5.6 The total $\omega$ yield measured from $3 \omega$ sample, cross section normal-

ization factors and total $\omega$ photoproduction cross section. The acceptance assumes an exponential t-distribution with a slope $b=$ $5.5 \mathrm{GeV}^{-2}$ this cross section includes contribution from $\Delta$ and $\mathrm{N}^{*}$ resonances as well as diffractive $\omega$ p production. . . . . . . . . . 66 


\section{Chapter 1 INTRODUCTION}

The theory of strong interactions, known as Quantum Chromodynamics (QCD), is a non-Abelian gauge theory characterized by confinement and asymptotic freedom [2:3][27][46] which describes the strong force. QCD is a quantum field theory that describes the interaction between quarks and gluons. QCD has three types of charges known as color charge unlike Quantum Electrodynamics (QED) which has only one type of charge know as electric charge. Quarks are fundamental

particles and carry fractional charges of $+\frac{2}{3}$ e or $-\frac{1}{3} \mathrm{e}$ and also color charges. They come in six different flavors each having its own anti-particle known as antiquark. The gluon, a massless vector boson is the force carrier in QCD and it transmits the strong force between quarks and antiquarks. Unlike the photon, which is the force carrier in Quantum Electrodynamics, the gluon carries the color charge. Since gluons are colored, they like quarks cannot be observed in isolation due to confinement and thus only color singlet objects can be observed in nature. These color singlet objects are known as hadrons. Hadrons can be categorized into two groups: mesons, made up of even numbers of quark antiquark pairs, and baryons, made up an odd number of quarks, at least three. Other states that can also exist are hadrons with no valence quark content. These hadrons are known 
as glueballs while hybrids are made up of valence quarks and anti quarks with a gluonic degree of freedom. There is no known analytic solution to the quantum field equations of $\mathrm{QCD}$, but the spectrum appears to be described to a large extent by a simplified model called the constituent quark model (CQM). In the CQM, all mesons are bound states of quark-antiquark pairs and all baryons are three-quark bound states. This model does not explain confinement and has no explicit role for gluons. Instead, the interaction between the quarks is replaced with a static potential, and confinement is inserted by hand in that the potential rises linearly at large radius. The quark and antiquark in a CQM meson are both spin $\frac{1}{2}$ and can combine to a total spin $\mathrm{S}=0$ (antiparallel) or $\mathrm{S}=1$ (parallel). In addition to spin, there is also the orbital angular momentum of the quark antiquark pair, L, which when combined with $\mathrm{S}$, gives the total angular momentum J. Mesons are classified in $\mathrm{J}^{P C}$ multiplets, with parity $\mathrm{P}$ given by $(-1)^{L+1}$ and charge conjugation $\mathrm{C}$ given by $(-1)^{L+S}$. The light quarks, up, down and strange, can be combined with the three light antiquarks to form the lightest mesons. This leads to nine possible $\mathrm{q} \bar{q}$ combinations with the same $\mathrm{J}^{P C}$, known as a nonet. The nonet is further broken down into two groups, eight members of an octet and single member of a singlet, distinguished by their transformation properties under SU3(flavor).

There are three nonets with $\mathrm{J}=1$ in the CQM: $\mathrm{J}^{P C}=1^{--}, \mathrm{J}^{P C}=1^{+-}$, and $\mathrm{J}^{P C}=1^{++}$. The fourth possibility $\mathrm{J}^{P C}=1^{-+}$, is forbidden by $\mathrm{q} \bar{q}$ selection rules in the CQM. General arguments based on the full gluonic degrees of freedom 
Table 1.0.1: Quantum numbers for the two lightest $\mathrm{q} \bar{q}$ nonets.

\begin{tabular}{lllllll}
\hline & & $\mathrm{J}^{P C}$ & $(\mathrm{I}=1)$ & $(\mathrm{I}=0)$ & $(\mathrm{I}=0) \mathrm{s} \bar{s}$ & $\mathrm{~S} \neq 0$ \\
\hline $\mathrm{L}=0$ & $\mathrm{~S}=0$ & $0^{-+}$ & $\pi$ & $\eta$ & $\eta^{\prime}$ & $\mathrm{K}$ \\
& $\mathrm{S}=1$ & $1^{--}$ & $\rho$ & $\omega$ & $\phi$ & $\mathrm{K}^{*}$ \\
\hline
\end{tabular}

in QCD suggest that these so-called exotic $\mathrm{J}^{P C}=1^{-+}$mesons actually exist in nature, and the experimental search for these states is an active area of research at present [3.5]. Current understanding of QCD predicts a richer spectrum of mesons that includes exotic states, hybrids, glueballs and multi-quark states[42]. Gluonic excitations in the meson spectrum are predicted by Lattice QCD calculations. [10] The only negative parity $\mathrm{J}=1$ mesons that are known to exist are the $\mathrm{J}^{P C}=$ $1^{--}$multiplet, known as the vector mesons.

\subsection{Diffractive Scattering}

Diffractive scattering can be compared to the optical phenomena in which a beam of light encounters an obstacle, the wavelength of the beam of light should be much smaller than the obstacle dimensions. In optics, intensity of the diffracted light at small angles and large wave numbers is given by the equation below:

$$
I(\theta) \simeq I(0)\left(1-B k^{2} v^{2}\right)
$$

where $\mathrm{B}$ is proportional to the radius squared of the obstacle and $\mathrm{kv}$ is proportional to the momentum transfer. The intensity has a forward peak followed by other secondary maxima. A similar behavior is seen in diffractive scattering pro- 
cesses, the differential cross section depicting an exponential behavior at small $|\mathrm{t}|$

$$
\frac{d \sigma}{d t}=A e^{-b|t|}
$$

Where the slope parameter $\mathrm{b}$, is proportional to the square radius of the target scattering radius. The slope parameter $\mathrm{b}$, can obtained experimentally and has typical values of between $5-13 \mathrm{GeV}^{-2}$ at high energies.For large $|\mathrm{t}|$, the differential cross section drops rapidly, but not with an exponential behavior since other secondary peaks appear. In general, at high energies, diffractive scattering can be defined as a reaction in which no quantum numbers are exchanged. A good example is the case of meson photoproduction, the target scatters in its ground state and the scattered particle is a vector meson. Diffractive scattering processes do need to be elastic since the incoming and outgoing particles can have different masses. Characteristics of diffractive scattering:

(a) the total cross section is almost energy independent;

(b) the differential cross section as a function of $|\mathrm{t}|$ (momentum transfer) follows an exponential behavior.

\subsubsection{Regge Theory}

At high energies, vector mesons are produced by Regge exchanges which are described using Regge theory. In this theory, angular momentum 1 is not restricted to have integer multiples of $\hbar$ but is allowed to be a complex variable. The 
scattering amplitude, is analytically continued into the complex plane [16]. The scattering amplitude singularities found in the complex plane are known as Regge pole and depending on the angular momentum value, they can correspond to either bound states or resonances. A Regge trajectory can be defined as hadronic states with the same quantum numbers such as isospin, strangeness, and baryon number and appear to lie on the same line if the spin of the particles is plotted versus the mass squared. For example, for a given reaction one needs to determine which quantum numbers can be exchanged. Both the vector meson and the photon have, charge conjugation, $\mathrm{C}=-1$, the photon has Isospin 0 or 1 and the $\mathrm{G}$ parity quantum number can be positive or negative. Therefore exchanges can have both natural $\mathrm{P}=(-1)^{J}$ and unnatural parity $\mathrm{P}=-(-1)^{J}$. One can use beam asymmetry measurements to probe the difference between natural and unnatural parity. subsection 1.1 .1 shows the possible Regge trajectories that can be exchanged in photoproduction of a neutral strangeless vector meson on a proton target. Regge exchanges are organized in families, each family is denoted by the lowest spin member. For example, all particles with $\mathrm{I}=0, \mathrm{~J}^{P C}=(0,2,4, . .)^{-+}$ belong to the $\eta$ family, particles with $\mathrm{I}=1$ and $\mathrm{J}^{P C}=(0,2,4, . .)^{++}$belong to the $\mathrm{a}_{2}$ family. Photoproduction of a strange vector meson requires Regge families with strangeness. The lorentz invariant Mandelstam variables are defined as :

$$
s=\left(P_{1}+P_{2}\right)^{2}
$$


Table 1.1.2: $\quad$ Leading Regge trajectories

\begin{tabular}{ll}
\hline Regge trajectory & $\mathrm{I}^{G} J^{P C}$ \\
\hline $\mathrm{a}_{2}$ & $1^{-} 2^{++}$ \\
$\pi$ & $1^{-} 0^{-+}$ \\
$\mathrm{a}_{1}$ & $1^{-} 1^{++}$ \\
$\mathrm{f}_{2}$ & $0^{+} 2^{++}$ \\
$\eta$ & $0^{+} 0^{-+}$ \\
$\mathrm{f}_{1}$ & $0^{+} 1^{++}$ \\
\hline
\end{tabular}

$$
\begin{aligned}
& t=\left(P_{1}-P_{3}\right)^{2} \\
& u=\left(P_{1}-P_{4}\right)^{2}
\end{aligned}
$$

where $\mathrm{P}_{1}, \mathrm{P}_{2}$ are the four momenta of the incident particles, $\mathrm{P}_{3}, \mathrm{P}_{4}$ are the four momenta of the final state particles. The Lorentz invariant variable $\mathrm{s}$, is the center of mass energy, $t$ is the square of momentum transfer. For a particular reaction of interest, one needs to determine the dominant Regge trajectory being exchanged. A general simplified amplitude for a two body scattering process, in the limit of large s, for the case of a single regge exchanges can be written as:

$$
A \sim \beta(t) s^{\alpha(t)}
$$

where the $\alpha(\mathrm{t})$ function is known as the Regge trajectory, which is actually the location of regge pole of $\mathrm{A}$ and $\beta(\mathrm{t})$ is the residue function at that pole. The Regge trajectories are linear in t, but have different slope and intercepts .Using the optical theorem one can relate the imaginary part of the forward scattering 
amplitude to the total cross section:

$$
\sigma_{\text {tot }}=\frac{1}{s} \operatorname{Im}\left(\left.A\right|_{t=0}\right)
$$

Thus Regge trajectory contributes a term to the total cross section that behaves as

$$
\sigma_{\text {tot }} \sim s^{\alpha(0)-1}
$$

The trajectories listed in subsection 1.11 have intercepts less than 0.5 and therefore this leads to total cross sections that decrease with energy [[]]. To account for the asympotically constant seen experimentally at higher energies [II] and [26] introduced a trajectory with an intercept of 1.0 and with vacuum quantum numbers known as the Pomeron after I. Ya Pomeranchuck. Note that although the Pomeron and the $\mathrm{f}_{2}$ have the same quantum numbers, vacuum quantum numbers, the $\mathrm{f}_{2}$ is a mesonic trajectory having intercept of 0.5 while the Pomeron has an intercept of 1.08. At high energies, the Pomeron exchanges dominate vector meson production. The residue function is proportional to the radiative decay width $\Gamma \sim \beta^{2} p^{3}$ of vector meson decays and since the radiative decay width for the $\omega \rightarrow \pi^{0} \gamma$ decay is greater than the radiative decay width for the $\omega \rightarrow \eta \gamma$ decay therefore it is expected that the $\pi$ exchanges to dominate over the $\eta$ exchanges. Thus the exchanges dominate over the unnatural exchanges. Spin density matrix elements (SDMEs) and polarization observables can be used to provide and use- 
ful information on the relative contribution of the different Regge trajectories. [34] This theory is expected to work well for energies above the resonance region, that is roughly $\mathrm{s}>10 \mathrm{GeV}^{2}$.

\subsection{Radiative Decays of Vector Mesons}

Radiative decays of vector mesons, vector meson $\rightarrow$ pseudoscalar meson, are an important probe to study the quark content of mesons. This is because the coupling to the charges and spin can reveal the nature of the hadron constituents wave function and thus are important in distinguishing between excitations. Since radiative decays probe wave functions better than hadronic decays, they can be used to access final states that are not accessible to hadronic decays. Radiative decays are strong discriminator between the $1^{3} \mathrm{~S}_{1}$ and $1^{3} \mathrm{D}_{1}$ orbital excitations of vector mesons [14]. Radiative decays can be used to distinguish gluonic excitations from conventional excitations with the same $J^{P C}$. For example, in a hybrid the $1^{--}$the $q \bar{q}$ are in a spin singlet state while in a conventional $0^{-+}$state the pair are in a spin triplet state. The radiative decays of the vector meson $\phi(1020)$, to sclar meson $\mathrm{a}_{0}(980)$ and $\mathrm{f}_{0}(980)$ is important in understanding the nature of these scalar mesons. These two scalar mesons have the same mass and are close to the threshold and their tendency to decay strongly suggest they are bound states with $\mathrm{I}=0$ and $\mathrm{I}=1$ [47]. The radiative decays of the $\phi(1020)$ meson, could shed some light on the nature of these scalar meson whether they are ordinary states, molecule[14] or a multi- quark state state. 


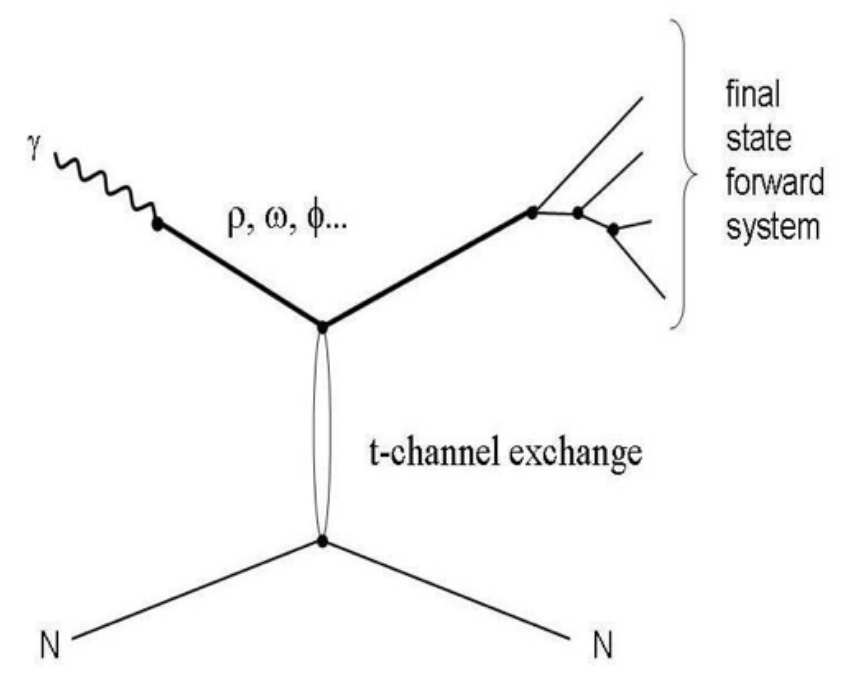

Figure 1.3.1: Representation of the VMD for the vector meson photoproduction mechanism with t-channel exchanges. The $\rho, \omega, \phi$ are vector mesons with $J^{P C}=$ $1^{--}$

\subsection{Vector Meson Photoproduction}

Photoproduction of vector mesons from hadrons has been an important tool to study Pomeron exchange at high energy and resonance exchanges at low energies[27]. The Vector Meson Dominance Model (VMD) assumes that photons interact with hadrons by first fluctuating into neutral vector mesons $\left(\left(\rho^{0}, \omega^{0}, \phi^{0}\right)\right)$ and then scatters peripherally from the target to produce the final state meson [38][39]. In this model the photon can be thought as a bare photon state undergoing purely electromagnetic interactions. Since the bare photon contribution to the interaction is very small compared to the contribution of the hadronic part. This behavior is depicted in section L.3 Thus the hadronic part of the photon can be modeled as a superposition of these vector meson states. VMD works under the assumption that these three mesons are the only hadronic constituents 
of the photon and that bare photon component does not interact with hadrons . The photon having the same quantum numbers as the vector mesons $\mathrm{J}^{P C}=1^{--}$ explain why a high energy photon beam is a excellent tool to study vector meson decays.

\subsection{Observables in Vector Meson Photoproduction}

To fully describe the production mechanism of vector mesons, $2 \times 2 \times 2 \times 3=24$ complex helicity amplitudes are required, this number is further reduced to 12 due to parity invariance. To determine all the 12 complex helicity amplitudes, a polarized beam, a polarized target and polarization measurements of the recoil nucleon are required. Unpolarized beam and target experiment such as Radphi, with beam energy $4.4-5.4 \mathrm{GeV}$, do not have the capabilities of measuring all the complex amplitudes but the observables measured such as spin density matrix elements and differential cross sections can be used to give an insight on the production mechanism of vector mesons such as $\omega(782)$ meson. 
Differential Cross Section: The differential cross section of a reaction is defined as the probability that the reactions occurs when the final state particles scatter into some final state kinematics. The differential cross section is a function of the reaction's final state paticle kinematic variable $X_{i}$. When no preferred polarization if produced,the cross section is referred to as an unpolarized differential cross section.

Total Cross Section: This is the probability that a reaction happens at some energy and is obtained by integrating the differential cross section over the final state kinematic variables.

Spin Density Matrix Elements: For particles with spin greater than zero, the probability that they are produced with a certain spin polarization can be determined by analyzing the angular distribution of its decay products.By analyzing the angular distribution of the decay products of the decaying particle, the relative probability of each spin polarization that is produced can be extracted.

\subsubsection{Previous Measurements}

Several studies of the photoproduction of (782) meson have been published. Ballam et al [4] in 1973 measured the differential cross section and spin density matrix elements using a hydrogen bubble chamber that was exposed to a linearly polarized photon beam energy at $4.7 \mathrm{GeV}$ at SLAC. The polarized photons were generated by laser backscattering. They were able to separate natural and unnatural exchange contributions by exploting the linear polarization of the beam. 


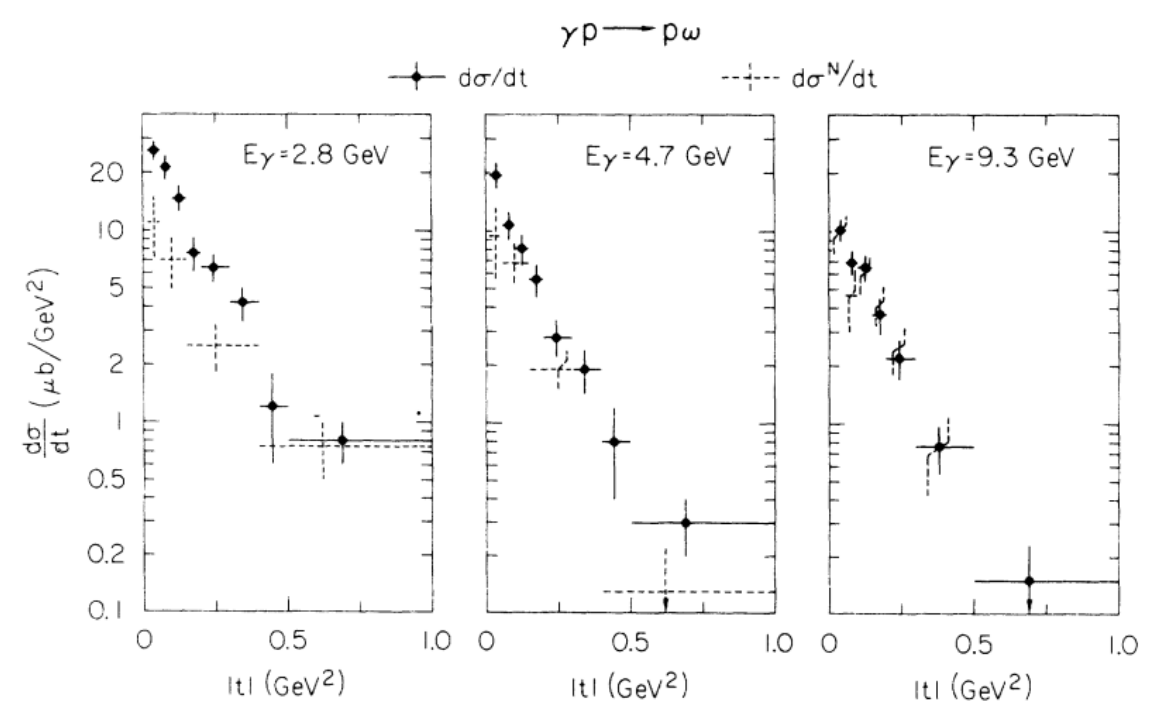

Figure 1.4.1: Differential cross section measured by SLAC,the solid circles are the differential cross sections while the dashed lines show the natural parity-exchange contribution to the differential cross-section.

The LAMP 2 collaboration [5] measured the differential cross sections and spin density matrix elements in the photon energy range $2.8<\mathrm{E}<4.8 \mathrm{GeV}$. The data used was obtained from the NINA electron synchrotron at Daresbury. They utilized a high resolution tagged photon beam incident on a hydrogen target. The omega mesons were detected through the decay . The charged particles were detected in the multi-wire proportional chambes and a lead glass calorimeter was used to detect the photons that reconstructed the They measure the spin density matrix elements in both Helicity and Gottfried Jackson frames. 


\subsection{Theoretical Predictions}

Daboul[17] utilizes a Regge pole model which includes $\mathrm{f}_{2}$ and $\mathrm{a}_{2}$ and Pomeron exchanges to describe the differential cross section and SDME. They do not include the pion trajectory because a pure One Pion Exchange(OPE) gives total cross sections 9 times larger for the $\omega$ than $\rho$ that is $\sigma_{\omega}: \sigma_{\rho}=9: 1$ which is in conflict with experimental data. They obtain smaller differential cross sections than experimental data and this could be due to the fact they negelct the pion trajectory contributions. The SDME obtained using this model are the natural parity contributions and since our data includes contributions from both natural and unnatural exchanges this model can will not describe our data since one needs a model that includes contributions from both natural and unatural parity exchanges. Oh, Titov and Lee[36] develop a model that incorporates a $\pi^{0}+\eta$ + Pomeron exchanges in the t-channel. they also include nucleon exchange in both the s and $\mathrm{u}$ channels. The OTL model follows the work of Donnachie and Landshoff [20] when constructing the Pomeron amplitude. The Parameters used in the Pomern exchange amplitude were determined by fitting the total cross sections for all vector meson $(\omega, \rho$ and $\phi)$ at high energies.

$\mathrm{Yu}$ and $\operatorname{King}[48]$ used an approach where they incorporate a $\pi(135)+\sigma(500)$ $+f_{1}(1285)+f_{2}(1270)+$ Pomeron exchanges in the $\mathrm{t}$ - channel at forward angles .The $\sigma$ exchange was added to reproduce the natural parity cross section. They also include Regge cuts so as to regulate the the strong contribution of the pion 
exchange.They compare their cross sections (differential and total) and SDME to available experimental data.

\subsection{Summary}

The primary goal of the Radphi experiment (E94-016 at Jefferson Lab) was to measure the radiative decays of the $\phi$ meson to all neutral states, $\phi \rightarrow M \gamma$, where $\mathrm{M}$ is a pseudoscalar meson such as $\pi^{0}$ or $\eta$, or a scalar meson such as $\mathrm{f}_{0}(980)$ or $\mathrm{a}_{0}(980)$. The decays involving the scalars are of special interest since they offer a unique insight into the quark substructure of these scalar mesons. Before searching for these rare radiative decays, dominant vector radiative decays such as the $\omega \rightarrow \pi^{0} \gamma$ were studied in order to normalize the detector response. The Radphi sample contains approximately $2 \mathrm{M} \omega$ events in this decay channel, which provides sufficient statistics to measure its spin density matrix elements. In this thesis, the spin density matrix elements (SDME) in the Gottfried Jackson (GJ) and Helicity frames and differential cross section for $\omega$ photoproduction from a nuclear target $\left({ }^{9} \mathrm{Be}\right)$ with the final state identified by triggering on the recoil proton are measured as functions of momentum transfer $|t|$ in the energy range $4.4-5.4 \mathrm{GeV}$. The $\omega$ is reconstructed from the neutral decay.

$$
\omega \rightarrow \pi^{0} \gamma \rightarrow \gamma \gamma \gamma
$$




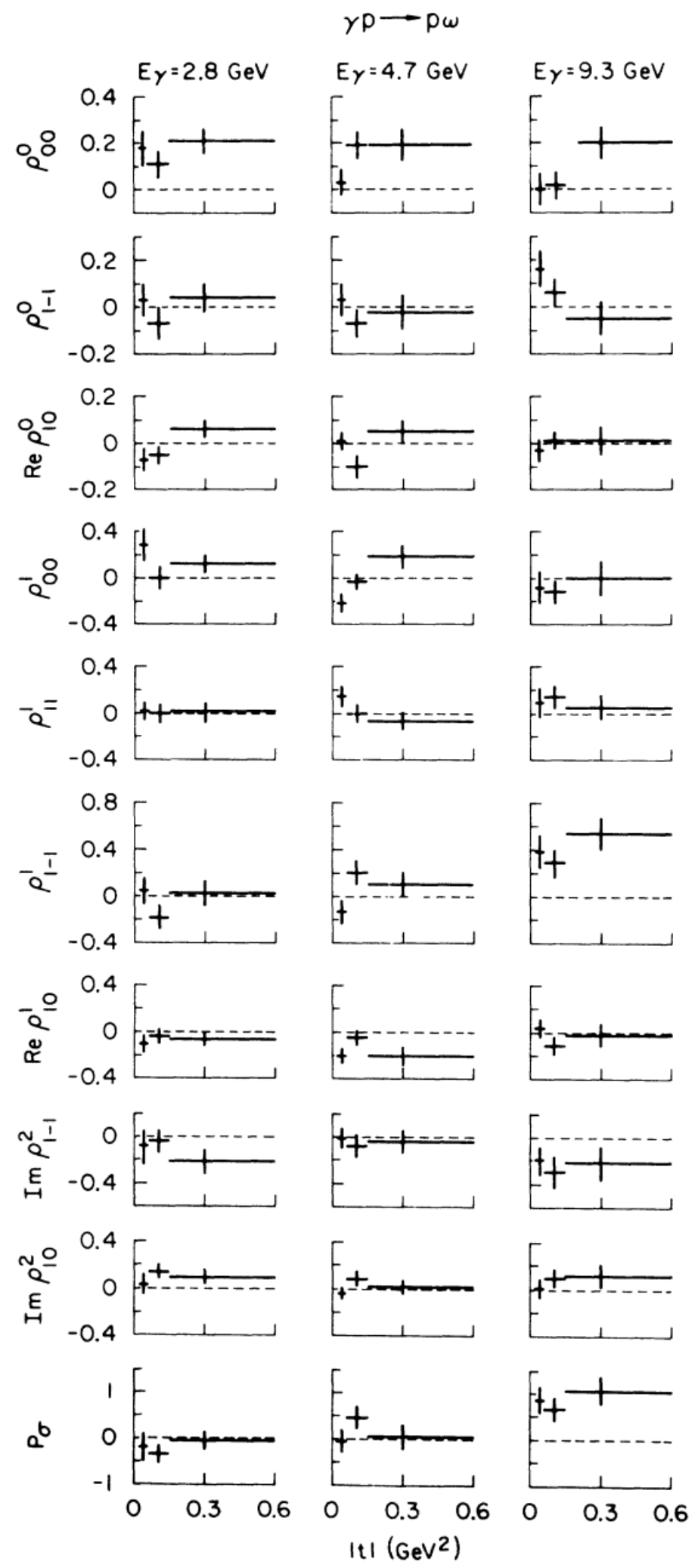

Figure 1.4.2: Spin denisty matrix elements and parity assymetry elements, in the helicity frame vs $|\mathrm{t}|$ from SLAC [G] 


\section{Chapter 2}

\section{Spin density matrix elements in $\omega$ photoproduction}

In quantum mechanics, the state of a quantum system may be formulated by specifying either a state vector that belongs to the Hilbert space or by a hermitian operator, the density matrix $\rho$, acting on the Hilbert state. The density operator formalism has the advantage of describing both pure and mixed states. A pure state is characterized by a single wave function and satisfies the condition $\rho^{2}=$ $\rho$. Particles in a fully polarized beam of monoenergetic particles are in a pure spin state. A mixed state refers to statistical mixtures of pure states. A mixed state can arise when a system is part of a statistical mixture for which ensemble averages are known but not the exact properties of the individual systems. For example in the case of an unpolarized beam the total polarization is known but the exact state of an individual photon is unknown. Mixed states can also occur due to entanglement of a system of interest with a larger system, where the larger system might be a pure state itself. Thus the subsystem can be expressed as a probabilistic mixture of the pure states. The density operator $\rho$ can be used to 
describe a system that is a probability mixture of pure states.

$$
\rho=\sum_{i} P_{i}\left|\Psi_{i}\right\rangle\left\langle\Psi_{i}\right|
$$

The vector $\left\langle\Psi_{i}\right|$ represents a pure state and are orthogonal. $P_{i}$ is the probability of being in a pure state $\left\langle\Psi_{i}\right|$ and $\sum_{i} P_{i}=1$. The density matrix representation of the density operator is written as:

$$
\rho_{i j}=\sum_{i j}\left\langle\Psi_{i}|\hat{\rho}| \Psi_{i}\left|\Psi_{i}\right| \hat{\rho} \mid \Psi_{i}\right\rangle
$$

Important properties of the density matrix are:

(i) The density matrix is hermitian $\rho=\rho^{\dagger}$.

(ii) The density matrix has unity trace $\operatorname{Tr} \rho=1$.

(iii) The diagonal elements are positive semi-definite that and this holds in any unitarily related basis.

\subsection{Spin Denisty Matrix}

For a two state quantum system, the density matrix can be expressed as a simple $2 \times 2$ matrix that describes the spin state of a particle with spin $\frac{1}{2}$ or a photon that is a massless gauge boson. The photon density matrix is studied in the helicity basis. Helicity is defined as the projection of spin onto the direction of momentum. Unlike other spin 1 particles, the photon is massless and has helicity $\lambda_{\gamma}= \pm 1$

and can never have $\lambda_{\gamma}=0$. Consider the case of linearly polarized photons 
with momentum parallel to the z-axis and following the convention discussed by Schilling [40], the photon state vector can be written as:

$$
\left|\gamma_{\phi}\right\rangle=\frac{1}{\sqrt{2}}\left(e^{-i \phi}\left|\lambda_{\gamma}=+1\right\rangle-e^{i \phi}\left|\lambda_{\gamma}=-1\right\rangle\right)
$$

where $\phi$ is the angle between the polarization vector of the photon and the production plane. Using the properties of density matrices, the photon density matrix can be written as:

$$
\rho^{\gamma}=\frac{1}{2}(\mathbb{1}+\overrightarrow{\mathbf{P}} \cdot \vec{\sigma})
$$

where $\mathbb{1}$ is a $2 \times 2$ unit matrix and $\sigma_{i}$ are the Pauli matrices. The $\overrightarrow{\mathbf{P}_{\gamma}}$ specifies the degree of polarization of the photon. For an unpolarized beam $\overrightarrow{\mathbf{P}_{\gamma}}=0$, for a circularly polarized beam $\overrightarrow{\mathbf{P}_{\gamma}}=P_{\gamma}(0,0, \pm 1)$, the \pm 1 corresponds to the helicity

of the photon. For linearly polarized beams, $\overrightarrow{\mathbf{P}}_{\gamma}=P_{\gamma}(-\cos 2 \phi,-\sin 2 \phi, 0)$ and $P_{\gamma}$ has values between $(0,1)$. Elliptical polarization can be obtained by the sum of linear and circular polarization.

\subsection{The $\gamma p \rightarrow \omega\left(\pi^{o} \gamma\right) p$ reaction}

In studying the $\gamma p \rightarrow \omega p$ reaction, we consider the case in which both the incident photon beam and target are unpolarized. Therefore we will average over the initial nucleon spins and avoid dealing with the extra notation that the nucleon spin density matrix would introduce. In an unpolarized photon beam experiment specified by $\rho^{\gamma}$ and averaging over the initial and final nucleon spins. We also 
make the simplifying assumption that the transition operator does not depend on the invariant mass of the $\omega$. Note that this approximation would hold if the $\omega$ meson had a width of 0 .

Defining our coordinate system in the overall center of mass (CM) frame the $\mathrm{z}$-axis is defined parallel to the photon momentum and the y-axis normal to the production plane.

$$
z=\frac{k}{|k|}, \quad y=\frac{k \times q}{|k \times q|}, \quad x=y \times z
$$

where $\mathrm{k}$ is defined as the photon momentum and $\mathrm{q}$ is the omega meson, $\omega$, momentum. Since the photon has no y component the omega meson production angle is only specified by the polar angle $\theta_{\omega}$. However in the case of a polarized photon beam, the polarization angle needs to be specified. Following the formalism described by Schilling [40], the vector meson density matrix $\rho(v)$ can be expressed in terms of the photon density matrix. The density matrices are connected by the transition matrix $\mathrm{T}$, which can be used to characterize photoproduction of vector mesons.

$$
\rho_{\lambda_{v}, \lambda_{v^{*}}}=\frac{1}{2} \sum_{\lambda_{N}, \lambda_{\gamma}, \lambda_{\gamma^{*}}} T_{\lambda_{v} \lambda_{\gamma} \lambda_{N}} \rho(\gamma)_{\lambda_{\gamma} \lambda_{\gamma^{\prime}}} T_{\lambda_{v}{ }^{\prime} \lambda_{\gamma^{\prime}} \lambda_{N}}
$$

where $\lambda_{V}, \lambda_{\gamma}, \lambda_{N}$ represent the helicities of the vector meson, incoming photon 
and the target nucleon, respectively. $\mathrm{N}$ is the normalization factor defined as:

$$
N=\frac{1}{2} \sum_{\lambda_{N}, \lambda_{\gamma}, \lambda_{v}}\left|T_{\lambda_{N} \lambda_{\gamma} \lambda_{v}}\right|^{2}
$$

The production amplitude normalization is chosen such that the unpolarized cross section is given by

$$
\frac{\mathrm{d} \sigma}{\mathrm{d} \Omega}=\left(\frac{2 \pi}{k}\right)^{2} \frac{1}{4} \sum_{\lambda_{N} \lambda_{\gamma} \lambda_{v}}\left|T_{\lambda_{N} \lambda_{\gamma} \lambda_{v}}\right|^{2}
$$

The photon spin density matrix can be decomposed and written in terms of the polarization vector $\mathrm{P}_{\gamma}$, therefore the four component spin density matrix can be expressed as .

$$
\left(\rho^{o}, \rho^{i}\right)=T\left(\frac{1}{2} \mathbb{1}, \frac{1}{2} \sigma^{i}\right) T^{\dagger}
$$

Where $\rho^{o}$ is the unpolarized component of the spin density matrix and $\rho^{i}$ is the polarized component. Therefore we can decompose the vector meson density matrix and show its explicit dependence on photon polarization as

$$
\rho^{v}=\rho^{0}+\sum_{i=1}^{3} P_{\gamma}^{i} \rho^{i}
$$

Where $\overrightarrow{\mathbf{P}_{\gamma}}$ parametrizes the incident photon beam density matrix $\rho^{\gamma}$. Writing these out explicitly we obtain:

$$
\rho_{\lambda_{v} \lambda_{v^{\prime}}}^{0}=\frac{1}{2 N} \sum_{\lambda_{\gamma} \lambda_{N}} T_{\lambda_{v} \lambda_{\gamma} \lambda_{N}} T_{\lambda_{v^{\prime}} \lambda_{\gamma} \lambda_{N}}^{*}
$$




$$
\begin{gathered}
\rho_{\lambda_{v} \lambda_{v^{\prime}}}^{1}=\frac{1}{2 N} \sum_{\lambda_{\gamma} \lambda_{N}} T_{\lambda_{v} \lambda_{-\gamma} \lambda_{N}} T_{\lambda_{v^{\prime}} \lambda_{\gamma} \lambda_{N}}^{*} \\
\rho_{\lambda_{v} \lambda_{v \prime}}^{2}=\frac{1 i}{2 N} \sum_{\lambda_{\gamma} \lambda_{N}} \lambda_{\gamma} T_{\lambda_{v} \lambda_{-\gamma} \lambda_{N}} T_{\lambda_{v^{\prime}} \lambda_{\gamma} \lambda_{N}}^{*} \\
\rho_{\lambda_{v} \lambda_{v},}^{3}=\frac{1}{2 N} \sum_{\lambda_{\gamma} \lambda_{N}} \lambda_{\gamma} T_{\lambda_{v} \lambda_{\gamma} \lambda_{N}} T_{\lambda_{v^{\prime}} \lambda_{\gamma} \lambda_{N}}^{*}
\end{gathered}
$$

Note that due to the definition of the normalization factor $N, \operatorname{Tr} \rho^{0}=1$. In our choice of coordinate system, the omega production reaction takes place in the $\mathrm{x}-\mathrm{Z}$ plane. Using the formalism of Chung[1:3] the consequence of the parity operator $\Pi_{y}$ on the transition matrix, $\mathrm{T}$, will leave the particles momenta unchanged and act directly on the rest state since all the momentum vectors lie on the $\mathrm{x}-\mathrm{z}$ plane. Thus the parity operator will change the helicitiy only.

$$
\Pi_{y}\left|s_{i} \lambda_{i}\right\rangle=\eta_{i}(-)^{s_{i}-\lambda_{i}}\left|s_{i}-\lambda_{i}\right\rangle
$$

where $\eta_{i}$ are the intrinsic parities of the particles involved and $\mathrm{s}_{i}$ are the spins involved. Therefore parity conservation reduces the spin density matrix elements to

$$
\begin{gathered}
\rho_{\lambda \lambda^{\prime}}^{i}=(-)^{\lambda-\lambda^{\prime}} \rho_{-\lambda-\lambda^{\prime}}^{i} \quad i=0,1 \\
\rho_{\lambda \lambda^{\prime}}^{i}=-(-)^{\lambda-\lambda^{\prime}} \rho_{-\lambda-\lambda^{\prime}}^{i} \quad i=2,3
\end{gathered}
$$


In this analysis we focus on the unpolarized component of the density matrix since the incident beam was unpolarized. The omega meson being a spin 1 particle, the possible spin polarizations are $-1,0,1$. Therefore the unpolarized spin density matrix can be expressed as

$$
\rho^{0}=\left(\begin{array}{ccc}
\rho_{-1-1}^{0} \rho_{0-1}^{0} \rho_{1-1}^{0} \\
\rho_{-10}^{0} & \rho_{00}^{0} & \rho_{10}^{0} \\
\rho_{-11}^{0} & \rho_{01}^{0} & \rho_{11}^{0}
\end{array}\right)
$$

However, due to the fact that the $\rho^{0}$ has to be hermitian, using the trace property of the matrix and from parity conservation the $\rho^{0}$ matrix can be expressed in terms of 3 real numbers.

$$
\rho^{0}=\left(\begin{array}{cc}
\frac{1}{2}\left(1-\rho_{00}^{0}\right) R e \rho_{10}^{0}+i m \rho_{10}^{0} & R e \rho_{1-1}^{0} \\
\rho_{00}^{0} & -R e \rho_{10}^{0}+i m \rho_{10}^{0} \\
& \frac{1}{2}\left(1-\rho_{00}^{0}\right)
\end{array}\right)
$$

where the bottom half of the matrix can be obtained by hermitian conjugation .

\subsection{Decay Angular Distributions}

The density matrix elements are important as they can be used to give information about the production mechanism of the omega meson. Thus the density matrix elements are extracted from the angular distributions of the final state vector meson decays [49]. The angular distribution of $\omega \rightarrow \pi^{0} \gamma$ in terms of the vector 
meson spin density matrix can be expressed as:

$$
W(\cos \theta, \phi)=M \rho(V) M^{\dagger}
$$

$\mathrm{M}$ is the decay amplitude of the omega meson, $\theta$ and $\phi$ are the polar and azimuthal angles of the decay products defined in the rest frame of the omega meson.

$$
\frac{\mathrm{d} N}{\mathrm{~d} \cos \theta d \phi}=W(\cos \theta, \phi)=\frac{3}{4 \pi} \sum_{\lambda, \lambda^{\prime}} D_{\lambda, \lambda_{a b}}^{1 *}(\Phi, \Theta,-\Phi) \rho_{\lambda, \lambda^{\prime}} D_{\lambda^{\prime}, \lambda_{a b}}^{1}(\Phi, \Theta,-\Phi)
$$

For $\omega \rightarrow \pi^{0} \gamma, \lambda_{a b}=\lambda_{a}-\lambda_{b}=0,1,-1$. The $\mathrm{D}_{m, m^{\prime}}^{j}(\mathrm{R})$ are the Wigner D-functions, which give the probability that the quantum state $|j, m\rangle$ is transformed into $\left|j, m^{\prime}\right\rangle$ after rotation R. Following the convention of Rose [3], the Wigner D-functions

$$
D_{m, m^{\prime}}^{j}(\alpha, \beta, \gamma)=e^{-i\left(m \alpha+m^{\prime} \gamma\right)} d_{m m^{\prime}}^{j}(\beta)
$$

where $\alpha, \beta, \gamma$ are Euler angles. Now the $\mathrm{W}$ function in Equation 2.3.2 can be decomposed using Equation 2.2.6

$$
W(\cos \theta, \phi)=W^{0}(\cos \theta, \phi)+\sum_{\alpha=1} P_{\gamma}^{\alpha} W^{\alpha}(\cos \theta, \phi)
$$

where $P_{\gamma}^{\alpha}$ is the photon polarization vector, the angles $\theta$ and $\phi$ specify the direction of one of the daughter particles from $\omega$ meson decay. For an unpolarized photon beam, only $W^{0}$ can be measured and thus only four independent observables can be measured, but the trace property reduces this number to three, with a linearly polarized beam six more observables can be measured and with a circu- 
larly polarized beam two more observables can be measured. The $\omega$ meson decay angular distribution (in this thesis it is the $\pi^{0}$ angular distribution) is defined as:

$W^{0}(\cos \theta, \phi)=\frac{3}{8 \pi}\left(\sin ^{2} \theta \rho_{00}^{0}+\left(1+\cos ^{2} \theta\right) \rho_{11}^{0}+\sin ^{2} \theta^{2} \cos 2 \phi \rho_{1,-1}^{0}+2 \sin 2 \theta \cos \phi R e \rho_{10}^{0}\right)$

where $\theta$ and $\phi$ are the angles of the decay pion measured in $\omega$ rest frame. Equation 2.3.5 is the defining equation for extracting the SDME from the Radphi dataset. From Equation 2.2.7 helicity conservation happens when $\lambda_{\gamma}=\lambda_{V}$. The spin density matrix elements are a measure of the interference between the independent transition amplitudes. The element $\rho_{00}^{0}$ measures the intensity of helicity flip at the $\gamma \mathrm{V}$ vertex while the intensity of the interference of the non-flip and double spin flip amplitudes can be measured by the $\rho_{1-1}^{0}$ term. The element $\rho_{11}^{0}$ is not an independent term and is equal to $\frac{1}{2}\left(1-\rho_{00}^{0}\right)$.

\section{Nuclear targets}

The Radphi experiment utilized a nuclear target, the reaction is specified ${ }^{9} \operatorname{Be}(\gamma, p \omega)^{8} \mathrm{Li}$. The detection of the proton in the final state allows us to assume quasi-eastic proton scattering, this is different from a hydrogen target in that the proton in the ${ }^{9} \mathrm{Be}$ nucleus is not at rest. Using energy and momentum conservation, this effect is taken into account when obtaining the angular distributions. Since this is a small nucleus we do not expect significant differences from a free proton target.

\subsection{Reference Frames}


There are many coordinate systems in which the polar and azimuthal angles of the decay products can be measured. They all differ by the choice of the z-axis which defines the quantization axis.

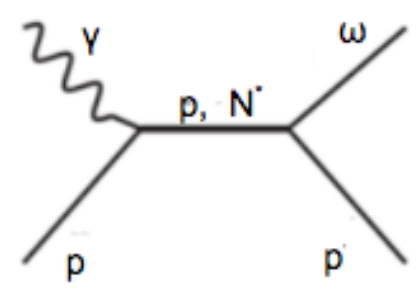

(a)

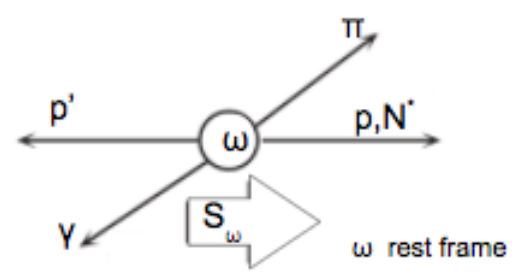

(b)

Figure 2.4.1: Diagrammatic representation of the helicty frame in the $\omega$ frame.

(i) Gottfried Jackson (GJ) frame: The Gottfried Jackson frame also known as the t-channel frame, is a frame where the $\mathrm{z}$-axis is chosen as the direction of the incident photon in the omega meson rest frame, and the $\phi=0$ half-plane as containing the direction of the final-state recoil nucleon.

(ii) Helicity frame: In the Helicity Frame, the z-axis is chosen as opposite the direction of the recoiling nucleon in the omega meson rest frame, and the $\phi=0$ half-plane as containsing the direction of the incident photon.

(iii) The Adair Frame: In the Adair Frame, the $\mathrm{z}$-axis is chosen as the direction of the photon in the center of mass frame.

In each of these reference frames, the y-axis is defined as in equationEquation 2.2.1. 


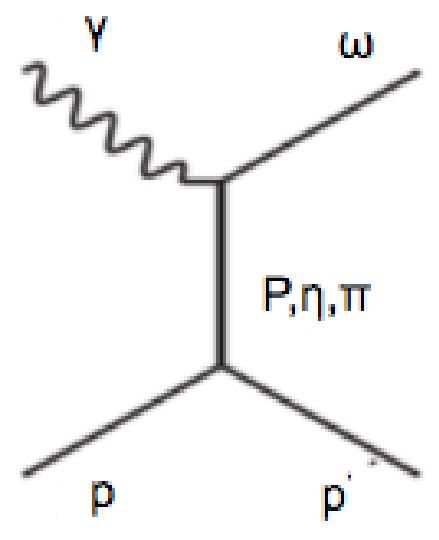

(a)

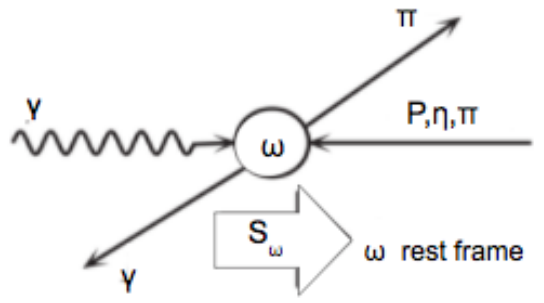

(b)

Figure 2.4.2: Diagrammatic representation of the Gottfried Jackson frame in the $\omega$ frame.

In both the helicty and Gottfried Jackson frame, the omega meson is at rest. The main difference between these two frames is the choice of the z-axis. The GJ frame is mainly used to study t-channel exchanges. In the t channel, if there is helicity carried by the exchange particle, the vector meson $\omega$ has helicities \pm 1 equal to the incident photon helicity. This condition is known as t-channel helicity conservation. The helicity channel is used to test s-channel helicity conservation, where the scattering amplitude vanishes when helicity of the initial photon differs from the z-projection of the final vector meson spin onto the z-axis. These two frames are connected by a rotation about the y-axis. Thus rotating by an angle $\alpha$, the angle between the z-axis in the helicity frame and the incoming photon 3-momentum vector, about the y-axis puts you in the Gottfried Jackson frame. Thus the spin density matrix elements depend on the choice of reference frame. 
In this analysis, the spin density matrix elements are extracted in both the GJ and the helicity frame.

\subsection{Helicity Conservation}

\subsection{1 s-channel helicity conservation}

One key assumption in vector meson photoproduction concerns the conservation of the helicity of the particles involved. That is the helicity of the photon would be retained by the outgoing vector meson. In terms of helcity amplitudes this can be written as

$$
T_{\lambda_{V}, \lambda_{N^{\prime}}, \lambda_{\gamma}, \lambda_{N}}=T_{\lambda_{V}, \lambda_{N^{\prime}}, \lambda_{\gamma}, \lambda_{N}} \delta_{\lambda_{V}, \lambda_{\gamma}} \delta_{\lambda_{N^{\prime}}, \lambda_{N}}
$$

And if we adopt the s-channel helicity conservation picture and neglecting the nucleon helicities, all the amplitudes corresponding to helicity change vanish.

$$
T_{01}=T_{10}=T_{0-1}=T_{-10}=T_{-11}=T_{1-1}=0
$$

Due to parity,the number of independent amplitudes reduces to 3 . Therefore the angular distribution in the case of s-channel helicty conservation becomes

$$
W^{0}(\cos \theta, \phi)=\frac{3}{8 \pi}\left(1+\cos ^{2} \theta\right) \rho_{11}^{0}
$$

\subsection{2 t-channel helicity conservation}

If the scattering proceeds with no flip of helicities in the t-channel, that is the 
photoproduced vector mesons having helicity the same helicity as the incoming photon, then we consider t-channel helicty to have been conserved. The angular distribution, $\mathrm{W}(\cos \theta, \phi)$ in this case retains the same form as Equation 2.5.3. The only difference being the angles used to define the angular distribution function. In general if there is no flip of t-channel helicities, one expects $\rho_{00}^{G J}=\rho_{10}^{G J}=\rho_{1-1}^{G J}$ $=0$ while no flip of s-channel helicities would mean that $\rho_{00}^{H}=\rho_{10}^{H}=\rho_{1-1}^{H}=0$, where these two frames differ by choice of quantization as discussed above. 


\section{Chapter 3}

\section{Experimental Apparatus}

The data used in our analysis was from the Radphi dataset taken at the Thomas Jefferson National Accelerator Facility (TJNAF) in Newport News,Virgina. The goal of the experiment was to study the radiative decays of $\phi$ meson to scalar meson such as the $\mathrm{a}_{0}(980)$ and $\mathrm{f}_{0}(980)$. The decays of the $\phi$ to these scalar meson are o interest since the ratio of their branching ratio could give an insight into their quark sub structure. However the experiment also provided high statistics datasets for other final states including the p. The detector used for Radphi was optimized to detect neutral final states. Over 1 TB of data was recorded and used for offline analysis.In this chapter, we discuss the selection procedure used to select the $\omega$ sample from the data with emphasis on selecting diffractive $\omega^{6} \mathrm{~s}$.

\subsection{CEBAF Continuous Electron Beam Accelerator Facility}

The Continuous Electron Beam Facility (CEBAF) which is now capable of accelerating electrons upto $12 \mathrm{GeV}$, though for the RAdphi experiment the maximum energy was $6 \mathrm{GeV}$.The electron beam is injected to the four experimental halls,Halls A,B, C and D (During the $6 \mathrm{GeV}$ era only Halls A,B and C were in 
operation). Since the Radphi dataset was obtained during the $6 \mathrm{GeV}$ era, we discuss the the accelerator configuration during that era.CEBAF consists of a pair of superconducting RF linear accelerators connected by 5 recirculation arcs in a race-track configuration. These superconducting radio-frequency cavities ensure that the electron beam is continuous with a high average current. That is it can accelerate electrons in bunches separted in time by $2 \mathrm{~ns}$. The injector provides the initial acceleration to the electron beam, the beam then enters the racetrack shaped main accelerator which consists of two straight segments and two semi-circular segments. For Radphi experiment, the electron beam accel-

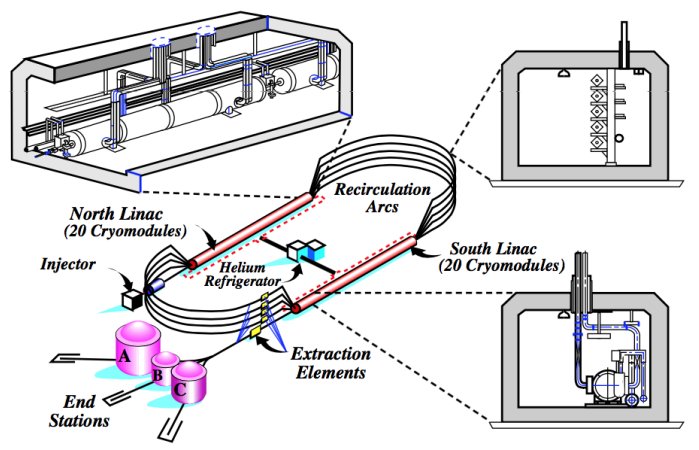

Figure 3.1.1: Schematic diagram of CEBAF during the $6 \mathrm{GeV}$ era

erator delivered a high duty factor electron beam of energy $5.65 \mathrm{GeV}$ to the photon tagging facility located in Hall B.The electron beam incident on a gold foil produced the photon beam via bremsstrahlung radiation. Since gold has a high atomic number this helped in the reduction of contamination of photons produced by electron-electron scattering. A radiator of thickness $2 \times 10^{-4}$ radiation lengths was used for Radphi experiment, the thickness was later increased 


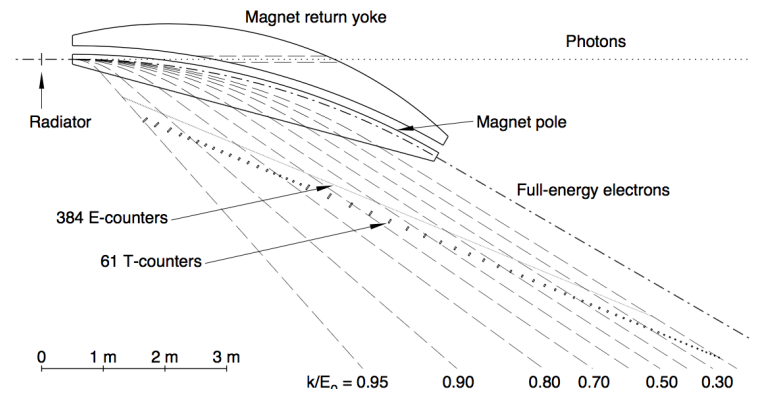

Figure 3.1.2: Scematic diagram of the tagger spectrometer. Dashed lines represent the trajectories taken by the recoil electron after being deflected by the dipole magnet.

to $2 \times 10^{-4}$ radiation lengths due to concerns regarding the electron-beam dump heating. This ensured that the photon flux remained the same at a lower electron beam current. Individual energy of the photons was measured by analyzing the energy of post-bremsstrahlung electrons using the Hall B tagged photon system. A dipole magnet field was used to deflect off the post- bremsstrahlung electrons, these electrons were then detected in a hodoscope that contained two planar arrays of plastic scintillators that were used to measure their energy. That is by by knowing the magnetic field, the electrons curvature can be determined and hence its 3-momentum can be reconstructed.Neglecting energy transferred to the gold nucleus, the energy of the photon can be determined by energy conservation.

$$
E_{\gamma}=E_{o}-E_{e}
$$

where $\mathrm{E}_{o}$ is the energy of the incident electron from the accelerator, $\mathrm{E}_{e}$ is the energy of the post-bremsstrahlung electron and $E_{\gamma}$ is the energy of the photon. 
Details on photon tagging are discussed elsewhere [3]].

\subsection{The Radphi Detector}

The Radphi experiment at Jefferson Lab (JLab) was designed to trigger on allneutral final states produced in a solid beryllium target by a tagged bremsstrahlung beam in the energy range $4.4-5.5 \mathrm{GeV}$. The main components of the Radphi detector are shown in Figure 3.2.1

The experiment was bulilt in the alcove of Hall B at Jlab located $40 \mathrm{~m}$ downstream of the bremsstrahlung radiator and tagging spectrometer [45]. A helium tube filled a region between the tagger and the alcove to reduce beam interactions upstream of the detector. The experiment used only tagging counters 1-19 of the Jefferson Lab Hall B tagging spectrometer to tag photons with energies from 4.4$5.4 \mathrm{GeV}, 75 \%$ to $95 \%$ of the electron beam energy. Using a gold bremsstrahlung radiator of thickness of $3 \cdot 10^{-4}$ radiation lengths and a $80 \mu \mathrm{A}$ electron beam produced a beam of approximately $5 \times 10^{7}$ tagged photons per second in the Radphi energy range (4.4-5.4 GeV). Details of the detector setup and performance are provided elsewhere [30]].

\subsubsection{Lead Glass Detector}

The main component was the lead-glass detector (LGD), used to measure multiphoton final states produced in the forward direction. The LGD was assembled as a circular array of 620 lead-glass blocks with a hole of $2 \times 2$ blocks in the center for the unscattered beam to pass through to the photon beam dump. The lead glass. 


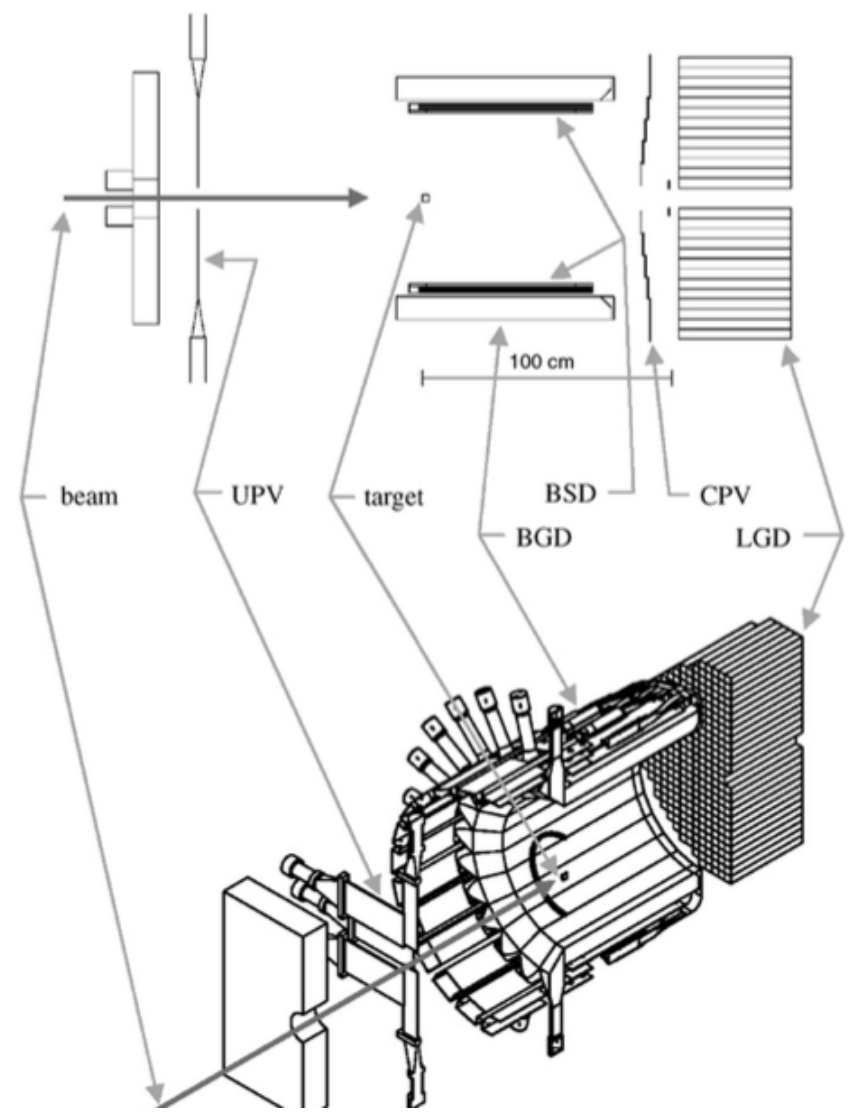

Figure 3.2.1: A cut-away view of the half of the Radphi detector. The beam enters from the lower-left passing through a hole in the lead shielding wall (foreground) and strikes the beryllium target (small disk at the center of the figure) which is suspended by thin wires from an aluminum ring. Surrounding the target are the barrel scintillator detector (BSD) and barrel gamma detector(BGD). In the forward region is the lead glass detector (LGD) covering polar angles $3^{0} 28^{0}$. Immediately in front of the LGD was a scintillator plane (notshown in the figure) which functioned as a charged particle veto (CPV). The scintillator array in the foreground is the upstream particle veto (UPV). Approximately $40 \mathrm{~m}$ upstream of the target was the photon tagger (not shown), where photons with energy between 4.4 and $5.4 \mathrm{GeV}$ were tagged

Each of the LGD blocks measured $4 \mathrm{~cm} \times 4 \mathrm{~cm} \times 45 \mathrm{~cm}$ with the long axis paralell to the beam. The lead glass blocks were stacked in $28 \times 28$ matrix, the corners of the matrix were removed to form an approximate circular configuration, the LGD covered lab polar angles from $3-28^{\circ}$ with good shower reconstruction capability 
within the range $4-24^{\circ}$.Each block had a PMT attached on the downstream end and the signals from PMT were used in the online trigger. The monitoring of the lead glass cells was accomplished with a pulsed nitrogen laser system that was used to illuminate the front face of the LGD wall. The laser monitoring system was useful for setup,debugging and also for the initial gain adjustement and relative gain monitoring of the LGD.

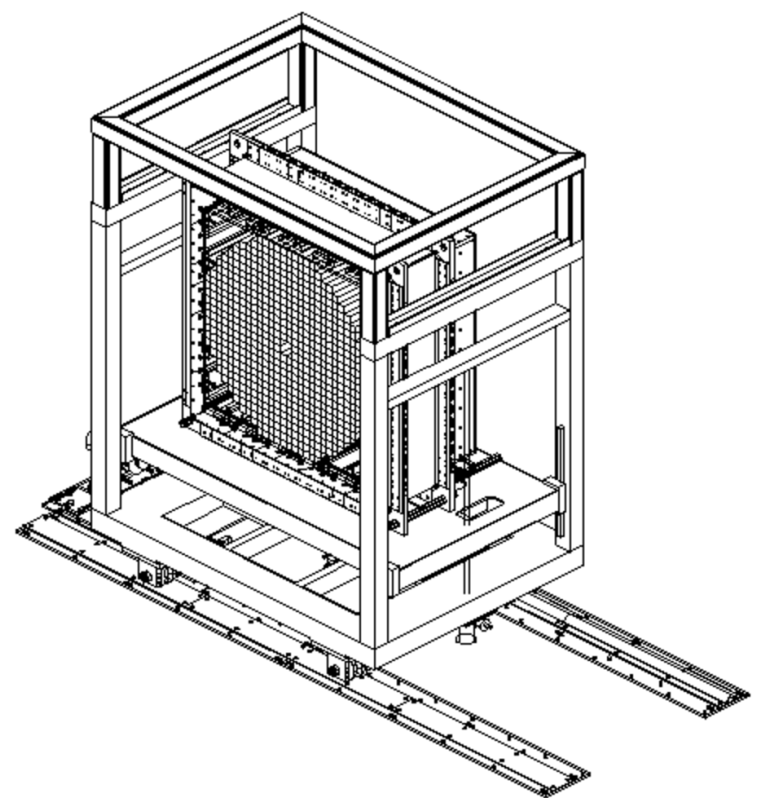

Figure 3.2.2: LGD mounted on its transponder.

The target was a beryllium cylinder of diameter $2.66 \mathrm{~cm}$ and length $2.54 \mathrm{~cm} . \mathrm{IT}$ was suspended and centered on the beam axis with 3 AWG steel wires from a $50 \mathrm{~cm}$ diameter Plexiglass ring. It was surrounded by a cylindrical scintillating detector,Barrel Scintillating Detector, (BSD) covering lab polar angles from $30^{\circ}$ to $110^{\circ}$ relative to the target and the full $360^{\circ}$ in azimuth. The BSD consisted of 3 concentric layers of scintillator paddles. The inner layer comprised of 12 
scintillator paddles that were identical and shaped like left handed helices that wrapped 1800 around the barrel cylinder. The middle layer consisted of 12 righthanded helical paddles similar to the inner layer. The outer layer consisted of 24 straight scintillator paddles. The main purpose of the BSD was to trigger on the recoil proton and measure the protons direction. The BSD was used to trigger on a recoil proton from -nucleus interactions, and the overlap information from hits in the three layers was used to determine the recoil proton direction. Radphi had no charged particle tracker and no magnetic field.

The BSD was surrounded by a cylindrical Barrel Gamma detector (BGD), which covered the same range in lab angles. The BGD composed of lead scintillating fiber matrix that were parallel to the beam axis and was used to veto photons that were produced from the target at large angles beyond the solid angle of the forward detector.The BGD was segmented into 24 counter with each counter having a read out at both ends Upstream of the target was a 10-cm thick lead shield wall with a $6.5 \mathrm{~cm}$ diameter hole for the photon beam. Just downstream of this wall was a 8-channel scintillator array (UPV) used to veto beam halo interactions inside the collimator.

A 30-channel scintillator array (CPV) covering the entire angular region in front of the LGD was used to veto charged particles in the final state.It was made up of 30 horizontal paddles , 15 each side of center, the paddles were organised in such a way that neighbouring paddles overlapped both vertically and at the center. The CPV scintialltors were $0.4 \mathrm{~cm}$ thick and were staggered around the plane at $\mathrm{z}$ 
$=90 \mathrm{~cm}$. High rates in the CPV prevented its use in the on-line trigger, so the vetoing of charged particles was only applied during the off-line analysis. The experiment employed a three-level trigger to select all-neutral final states. The level-1 trigger was a coincidence between the logical OR of the signals from the photon tagger and the logical AND of the three layers of the BSD. The UPV scintillators were also included as an anti-coincidence at level-1. In the level-2 trigger at least one of the calorimeter modules in the LGD was required to be above a minimum threshold, which was set at $1 \mathrm{GeV}$. The level-3 trigger required that the sum of all LGD modules be above the threshold, set at $3.0 \mathrm{GeV}$. These conditions for triggering were chosen so as to maximize the number of desired hadronic events while minimizing the number of background events. All events that passed level-3 were recorded to tape for further analysis.

Details on the detector components are discussed elsewhere [3T] 


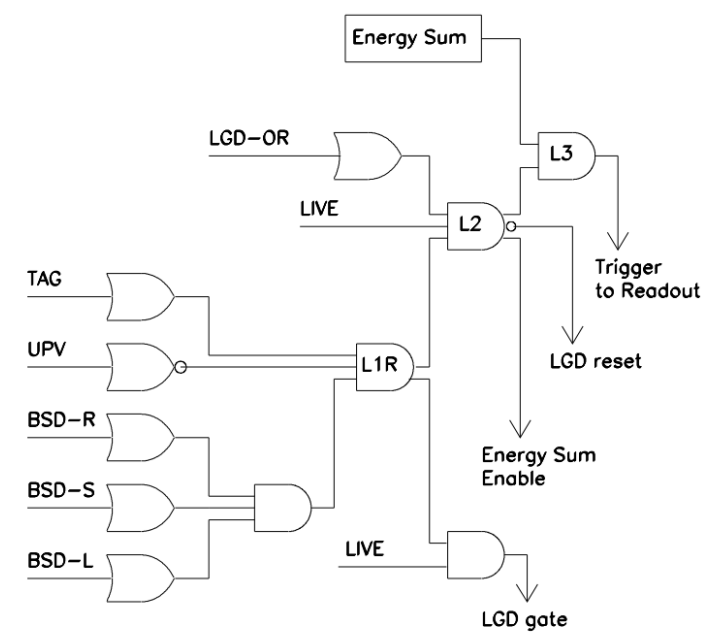

Figure 3.2.3: A schematic view of the Radphi 3-level trigger logic 


\section{Chapter 4}

\section{Data Analysis}

\subsection{Event Reconstruction}

In the off-line analysis the overlap geometry of the three BSD layers enabled a unique identification of a space point (pixel) along a track of a charged particle based upon a triple coincidence between the layers. After adjusting for light propagation, a pixel time was formed to estimate the start time for the event, which was used as a common reference time for all other detector components. A photon incident on the LGD deposits energy in several LGD blocks. The first step in event reconstruction was to go down the list of all blocks with energy above pedestal and to group adjacent blocks into clusters. The summed energy for each cluster and the cluster centroid were used to estimate photon energy and direction using a 2-dimensional non-linear response function that was derived from Monte Carlo (MC). Photon showers from $50 \mathrm{MeV}$ up to $5 \mathrm{GeV}$ were efficiently reconstructed in the LGD. Most of the photons below $50 \mathrm{MeV}$ consisted of a single LGD block and could not be distinguished from noise. In the case where two showers appear close together in the LGD, the energy of the blocks common to both clusters was shared using an algorithm that depended upon the distance 
of the block from each adjacent cluster and the cluster energies. The minimum cluster separation was $6 \mathrm{~cm}$, limited by the dimension of the LGD block of $4 \mathrm{~cm}$. Consequently, photons closer than $6 \mathrm{~cm}$ at the face of the LGD were merged into a single cluster. The LGD segmentation allowed this clusterizer to effectively reconstruct events of up to a shower multiplicity of 8 .

The initial calibration of the LGD was done by adjusting the high voltage of the LGD photo-tubes to equalize the response to an optical light pulse from the UV laser that was fanned-out to all of the blocks. Off-line, the energy calibration of the LGD was refined by constraining the $2 \gamma$ invariant mass in 2-cluster reconstructed events to match the physical mass of the $\pi^{0}$. In the class of events with two reconstructed showers the $\pi^{0}$ peak dominates the invariant mass spectrum. All events with an invariant mass within $\pm 30 \%$ of the $\pi^{0}$ mass were selected, and the global $\chi^{2}$ for the sample was computed reflecting the width of the $\pi^{0}$ peak. This $\chi^{2}$ has quadratic dependence on the individual gains of each LGD channel, permitting the $\chi^{2}$ to be minimized in respect to these gains by solving a linear equation. The adjusted gains were used in repeated passes through the reconstruction until the gain corrections converged to fixed values. This procedure led to a final $\pi^{0}$ peak whose width matched the one obtained by reconstructing simulated events with ideal calibration. The calibration was checked by examining other known all-neutral meson decays: $\eta \rightarrow \gamma \gamma$ in the 2-cluster sample, $\omega \rightarrow \pi^{0} \gamma$ in the 3 -cluster sample and $\eta \rightarrow 3 \pi^{0}$ in the 6 -cluster sample. The single-shower spatial and energy resolutions of the LGD extracted from the widths of the $\pi^{0}$ and 
Table 4.2.3: Table showing $\pi^{0}$ and $\omega$ decay modes and branching fractions

\begin{tabular}{lll}
\hline Meson & Decay Mode & Branching Ratio \\
\hline$\pi^{0}$ & $\gamma \gamma$ & $(98.823 \pm 0.034) \%$ \\
& $e^{+} e^{-} \gamma$ & $(1.174 \pm 0.035) \%$ \\
$\omega$ & $\pi^{+} \pi^{-} \pi^{0}$ & $(89.2 \pm 0.7) \%$ \\
& $\pi^{0} \gamma$ & $(8.28 \pm 0.28) \%$ \\
& $\pi^{+} \pi^{-}$ & $\left(1.53_{-0.13}^{+0.11}\right) \%$ \\
\hline
\end{tabular}

$\eta$ mass peaks were in good agreement with expectations for lead glass calorimeters.

\subsection{Event Selection}

The dataset used in this analysis was collected by the Radphi collaboration. Events of the type $\gamma p \rightarrow \omega p$ were selected from the Radphi dataset. Since the $\omega$ and $\pi^{0}$ mesons decays strongly,hence a very short lifetime, we observe them through their decay products.

The $\omega$ and $\pi^{0}$ decay modes are listed in the TableTable 4.2.3 The Radphi detector was optimized to detect neutral particles in the final state, therefore for this analysis the detectable modes for $\omega$ and $\pi^{0}$ are

$$
\omega \rightarrow \pi^{0} \gamma \operatorname{and} \pi^{0} \rightarrow \gamma \gamma
$$

Three neutral showers were reconstructed in the forward detector and a series of cuts applied to select exclusive $\gamma p \rightarrow \omega p$ events and reject events involving all other reactions. To estimate the number of events that were not detected, the final states were simulated using a Monte Carlo simulation program. After applying 
the data reduction cuts, there were still background events which could not be eliminated using the data reduction cuts. These events were further suppressed using the sideband subtraction technique.

\subsubsection{Selection of $\omega$ events}

The Radphi dataset consisted of $10^{9}$ tagged events collected in a run period of 430 hours of integrated live time. The integrated luminosity for tagged photon interactions during the experiment was $1.89 \times 10^{7} \mu b^{-1}$. Computing the target density assuming four protons per ${ }^{9} B e$ nucleus this corresponds to $7.56 \times 10^{7} \mu b^{-1}$ of $\gamma p$ interactions. Events were selected with three neutral showers in the forward detector, LGD. To optimize efficiency for peripheral $\gamma-p$ interactions the trigger and offline analysis were devised to select events with a single charged track at lab polar angle greater than $30^{\circ}$. A series of cuts were applied to the $3 \gamma$ sample to suppress electromagnetic and hadronic background and to select events with $\pi^{0} \gamma$ kinematics.

The following criteria were used in the offline analysis to select $\gamma p \rightarrow \omega p \rightarrow \pi^{0} \gamma p$ event candidates and to suppress background.

(i) $0.05 \mathrm{GeV}$ minimum shower energy in the LGD;

(ii) exactly three showers reconstructed in the LGD;

(iii) $3.0 \mathrm{GeV}$ minimum total shower energy in the LGD;

(iv) only one pixel cluster in the BSD; 
(v) no coincident hits in the forward veto scintillators (CPV);

(vi) no showers unrelated to the recoil reconstructed in the barrel gamma detector (BGD);

(vii) no low-energy showers around the beam hole in the LGD;

(viii) kinematic selection of $\pi^{0} \gamma$ events.

The signature of the $\omega$ meson in the detector is the identification of three neutral showers .It is expected that when the invariant mass distribution is plotted, a peak corresponding to $\omega$ prodcution will be seen at:

$$
M_{3 \gamma}=\sqrt{P_{\gamma 1}+P_{\gamma 2}+P_{\gamma 3}}
$$

where $P_{\gamma}$ is the four- momentum of the photons reconstructed in the forward detector. The minimum shower energy cut (1) suppresses electromagnetic background in the calorimeter. Cut 2 selects most of the $\omega \rightarrow \pi^{0}$ decays that appear in the Radphi sample. Cut 3 refines the on-line level-3 trigger energy threshold condition. Cut 4 applies a single recoil track requirement that corresponds to events with one-prong in the barrel region. An event is classified as n-prong if there are $\mathrm{n}$ charged tracks coming from the primary reaction vertex. Cut 4 , combined with the forward charged particle veto (5), rejects multi-prong reactions, which dominate the total photoproduction cross section at these energies. The barrel gamma veto (6) rejects events with large angle showers as well as suppressing 
several reactions with an excited baryon in the final state. The beam hole cut (7) is needed to reduce a contamination from uncorrelated photons around the beam hole that result from the electromagnetic interactions of the beam in the target. The kinematic selection of events with $\pi^{0} \gamma$ kinematics (8) further improves the signal-to-background ratio.

The charged particle veto cut (5) was very important because of the high rate of multi-prong reactions that dominate over the one-prong final states that are the focus of study in this analysis. The total rate observed in the CPV was a factor of about 300 larger than the calculated total photo-nuclear interaction rate in the target, indicating that that the CPV is dominated by electromagnetic backgrounds. Monte Carlo (MC) simulations were able to reproduce the observed rates in the CPV within 5\% based on electromagnetic interactions alone. Because of these high rates, dead-time considerations prevented the use of the CPV in the on-line trigger. The coincidence spectrum between the CPV and a BSD pixel is shown in the first panel of Figure 4.2.2. The peak near $\Delta t=0$ comes from CPV-BSD coincidences which sits on top of a large background coming from accidentals. The off-line veto window of 6 ns width, indicated by the dashed lines in the figure, was chosen as a compromise between veto efficiency (95\%) and signal loss from accidental vetoes $(32 \%)$.

. Electromagentic interactions in the beam in the target would result in uncorrelated photons around the beam hole, events that resulted from these interactios were effectively suppressed by applying a 2-D energy-angle correlated cut,cut 


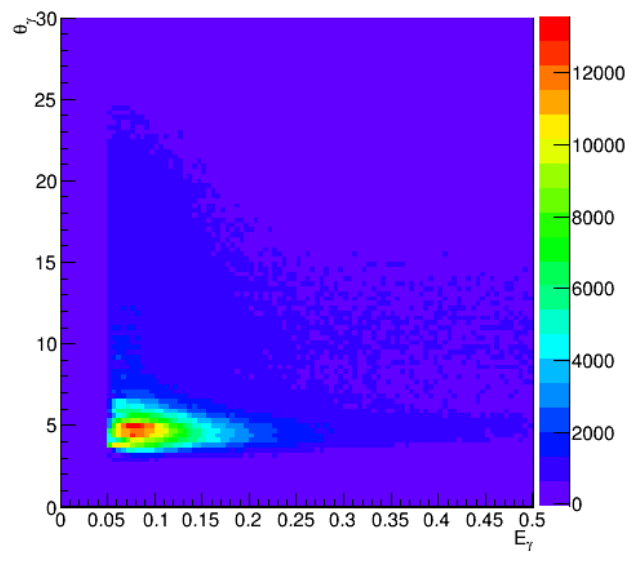

(a)

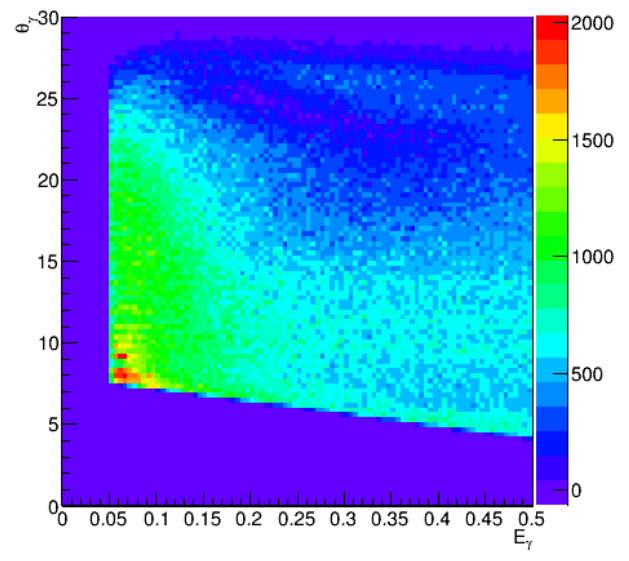

(b)

Figure 4.2.1: $3 \gamma$ polar angle measured in the lab frame versus total shower energy spectrum distribution (left panel). The distribution on the right panel shows the effect of cut 6

6.Figure 4.2.1 shows $3 \gamma$ polar angle plotted versus the total shower energy spectrum, events in the the lower part of the plot were excluded from further analysis. Showers with energy less than $0.5 \mathrm{GeV}$ were required to satisfy:

$$
E_{\gamma}+A \theta_{\gamma}>B
$$

with $A=0.13 \mathrm{GeV} /$ degree and $B=1.033 \mathrm{GeV}$. The values of $\mathrm{A}$ and $\mathrm{B}$ were chosen to reduce the observed electromagnetic background that peaks at low angles and energies. This cut combined with the other cuts described above further improves the $\omega$ signal-background ratio. To suppress an additional combinatorial background from $2 \gamma$ events that leak into the $3 \gamma$ sample and show up as a $\pi^{0}$ peak an additional cut on the invariant mass of the $2 \gamma$ sample was applied. This cut required that the two lightest photons from the 3 photon sample were required to 
have mass between $0.1-0.18 \mathrm{GeV}$. This cut also ensured that most of the events selected were compatible with a $\pi^{0} \gamma$.

High rates in the tagger made it impossible to identify a reconstructed event with a single tagged photon and therefore the treatment of accidental coincidences required special consideration. Since the tagger had a 20ns online coincidence window, for any true coincidence that was present for any given event, one accidental tagging coincidence was expected. The coincidence was further reduced to 6 ns after timing calibrations and signal propagation delays were done. Therefore, a neutral tagged spectrum was formed by requiring coincidence between a tagging counter and the BSD pixel that provided the reference time for an event and then subtracting the tagging accidentals. That is only the tagger hits that do not appear near the CPV could be used towards the accidental subtraction. The tagger coincidence was thus used to perform two functions, to remove beam photons outside the tagging range and to veto events with charged particles in the forward. This tagging information was saved as an event weight factor. It is expected that the mean energy from the total energy spectrum from the $3 \gamma$ sample corresponding to each tagging counter should be consistent with the tagger mid-point energy for that counter.Therefore looking at the total energy spectrum weighted with the above described weighting factor should reveal this behavior. The total shower energy distributions corresponding to each individual tagging counters are fitted with a gaussian and the mean obtained as a parameter of the Gaussian fit is compared with the mid-point tagger energy of each counter. The 


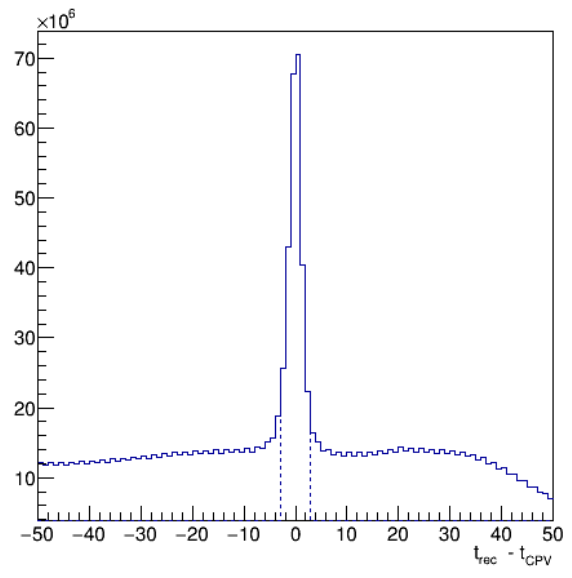

(a)

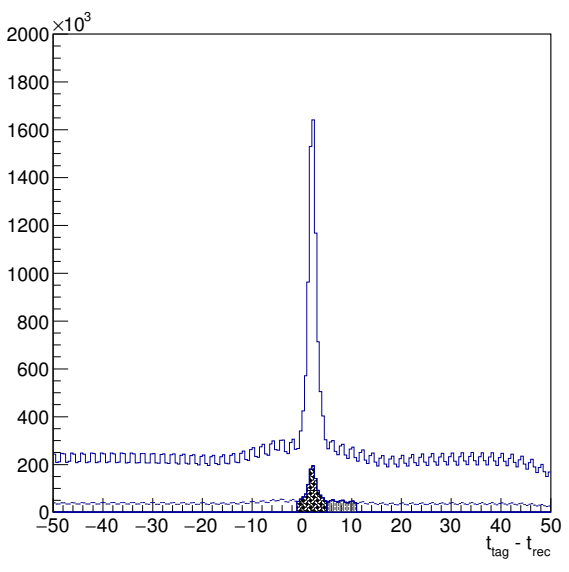

(b)

Figure 4.2.2: The time difference between a pixel and a hit in the CPV (left) and the tagger (right). Dashed lines in the left panel show the time window used for the charged particle veto. The right panel shows the tagger-pixel time difference before (solid) and after (dashed) the $\mathrm{CP}$ veto is applied. The shaded areas in the right panel show the time windows used for counting tagger coincidences (peak region) and accidentals (right of peak region).

method described above ensures that all neutral events in true association with tagged photons are selected.The distribution shown in 4.2 .3 is fitted with a first order polynomial and the slope of 1 reveals a good agreement between the expected energy in the forward and the measured energy after taking into account some energy that is transferred to the recoil proton. All events that did not meet the selection criteria described above, were eliminated from the analysis.Both the experimental data and simulated data were subjected to the cuts described above.

\section{Invariant Mass Spectrum After Initial Cuts}

There are $2.48 \cdot 10^{8}$ events that were reconstructed with three showers in the LGD.

The effects of various cuts on the $3 \gamma$ invariant mass spectrum are shown in Eigwre 4.2.4. The left plot in Figure 4.2.4 is obtained from the full sample after the 


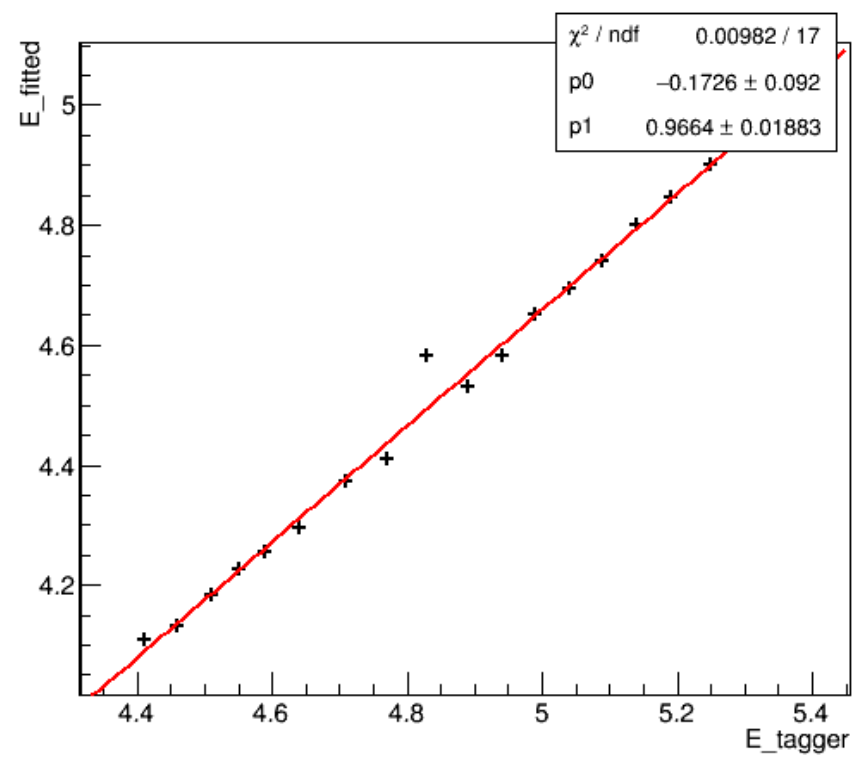

Figure 4.2.3: The mean energy obtained from fitting the tagged total shower energy distribution for each tagging counter plotted as function of mid point energy of each tagging counter.

application of cuts 1-4. The dashed line shows the effect of cut 5. The solid line in the right plot of Figure 4.2.4 shows the effects of cuts 5 and 6, while dashed line shows the effect of cut 7 . After these three additional cuts the peak around 0.8 $\mathrm{GeV} / \mathrm{c}^{2}$ that is associated with $\omega \rightarrow \pi^{0} \gamma$ stands out clearly above the background. The peak below $0.2 \mathrm{GeV} / \mathrm{c}^{2}$ arises from a solitary $\pi^{0}$ that feeds into the $3 \gamma$ sample by the addition of an accidental low-energy shower. Similarly, the peak around $0.6 \mathrm{GeV} / \mathrm{c}^{2}$ is associated with feed-through from a solitary $\eta \rightarrow 2 \gamma$ decay. MC simulations show that the enhancement around $0.35 \mathrm{GeV} / \mathrm{c}^{2}$ comes from leakage of multi-prong events into the sample, mainly $\rho \rightarrow \pi^{-} \pi^{0}$ and $\omega \rightarrow \pi^{-} \pi^{+} \pi^{0}$, where one of the charged pions deposits enough energy in the calorimeter to mimic a low energy shower. 
A method that is effective for reducing feed-through from $2 \gamma$ channels is to require that the invariant mass of the lightest photon pair in the final state falls in the region of the $\pi^{0}$ peak. Figure 4.2.5 shows the $2 \gamma$ invariant mass of all pairs from the $3 \gamma$ sample (solid line) and the lightest pair (dashed line). Accidental background showers tend to produce events with at least one photon pair with a mass less than $100 \mathrm{MeV} / \mathrm{c}^{2}$. Requiring the lightest pair to have a mass between 0.1 - $0.18 \mathrm{GeV} / \mathrm{c}^{2}$ (cut 8, shown by dotted lines in Figure 4.2.5) effectively suppresses those events while preserving essentially all of the $\omega \rightarrow \pi^{0} \gamma$ signal.

The last analysis step is to apply the tagging coincidence requirement and subtract tagging accidentals in order to subtract the irreducible background from the $3 \gamma$ sample and remain with a clean omega signal. The final. result for the $3 \gamma$ invariant mass spectrum is shown in Figure 4.2.6. The histogram is fitted with a double Gaussian above a broad smooth background (solid red curve). The double Gaussian represents the $\omega$ meson. The background is parametrized by a broad symmetric Gaussian peaked near $0.45 \mathrm{GeV}$ plus a second asymmetric Gaussian that peaks near $1 \mathrm{GeV}$. The sum representing the hadronic background is shown by the green curve. The $\omega$ yield from the fit, the peak position, the width, and the signal-to-background ratio are listed in the Table 4.2.4.

\subsection{Sideband subtraction}

After all the data reduction cuts discussed in section above have been applied, the invariant mass distribution still has a sizeable amount of background under- 


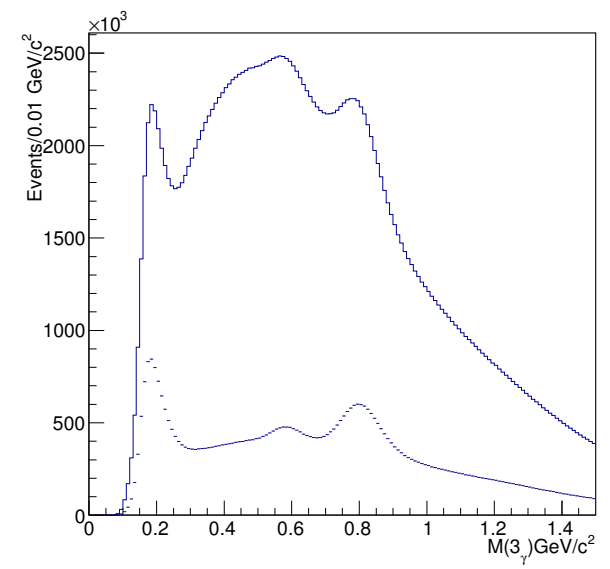

(a)

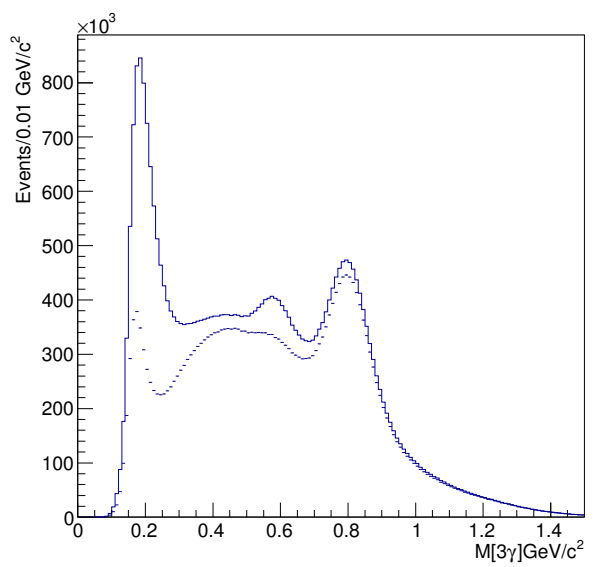

(b)

Figure 4.2.4: The invariant mass distribution from the $3 \gamma$ sample obtained after cuts 1-4 above (left panel solid curve), after cuts 1-5 (left panel dashed curve), after cuts 1-6 (right panel solid curve) and after cuts 1-7 (right panel dashed curve). The peak at $0.8 \mathrm{GeV} / \mathrm{c}^{2}$ (right panel) associated with $\omega \rightarrow \pi^{0} \gamma$ events stands out clearly above background.

Table 4.2.4: Results of fitting the $3 \gamma$ invariant mass distributions from the sample with 3 reconstructed showers. The reported errors are statistical only. The width of $53 \mathrm{MeV} / \mathrm{c}^{2}$ is dominated by the mass resolution of the detector. The centroid is shifted away from the physical mass at $0.782 \mathrm{GeV} / \mathrm{c}^{2}$ by approximately half this width due to a slight systematic bias in the energy calibration of the LGD based on the $\pi^{0}$ peak. No attempt was made to artificially correct for this bias.

\begin{tabular}{lll}
\hline Quantity & Value \pm Error & Units \\
\hline Yield $\left[10^{6}\right]$ & $1.71 \pm 0.09$ & \\
Centroid & $0.811 \pm 0.001$ & $\mathrm{GeV}$ \\
RMS Width & $0.0525 \pm 0.0003$ & $\mathrm{GeV}$ \\
signal/background & & \\
ratio & $5: 1$ & \\
\hline
\end{tabular}




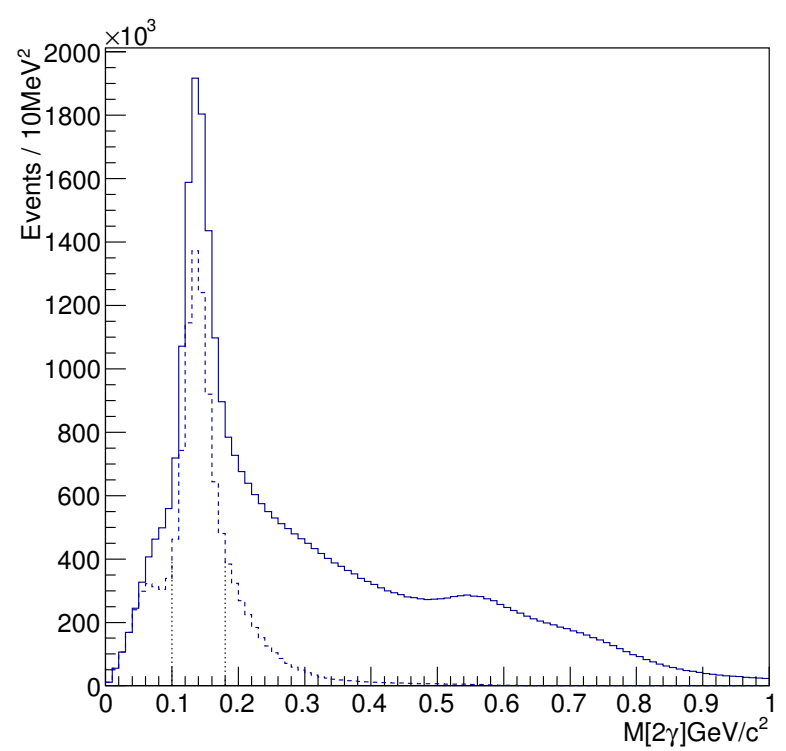

Figure 4.2.5: The invariant mass distribution of all photon pairs from the $3 \gamma$ sample (solid line). The dashed line corresponds to the lightest pair. The vertical dotted bars show the kinematic selection of the $\pi^{0}$.

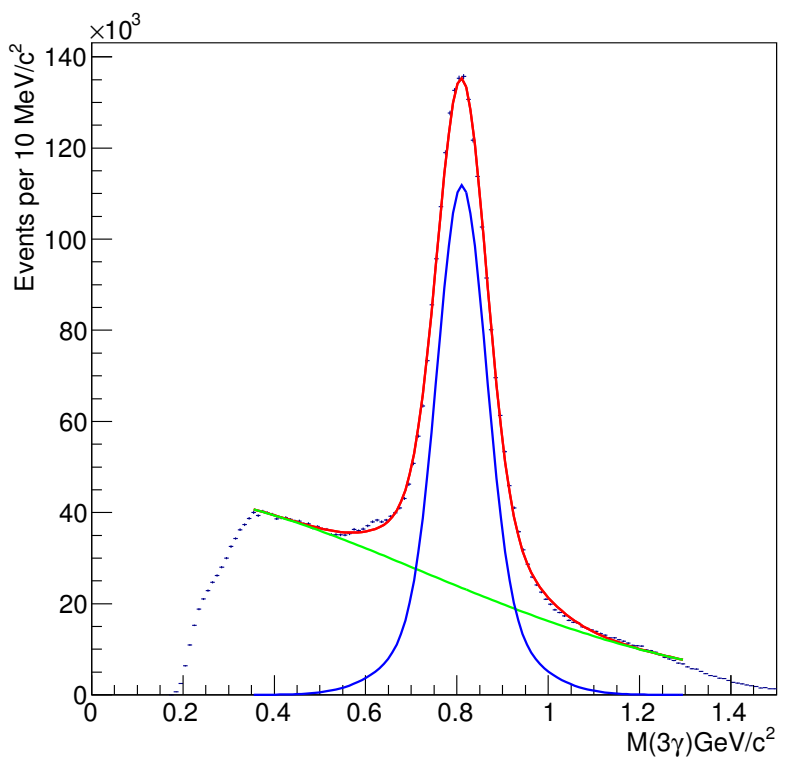

Figure 4.2.6: The $\pi^{0} \gamma$ invariant mass distribution for the final $3 \gamma$ sample after applying cuts 1-8 and applying the tagger accidental subtraction. The blue curve represents the signal while the green curve represents the background. 
neath the $\omega$ peak. To obtain kinematic distributions for events in which an $\omega$ is present, a fairly standard method of background subtraction is used to eliminate events under the invariant mass peak. Fig(..) shows a plot of the $3 \gamma$ invariant mass spectrum, the red region in the center includes an $\omega$ signal along with a background underneath the peak. To eliminate this background the method of sideband subtraction is used.

In this approach, we take the region that contains the peak and for events inside this peak region we assign them an event weight $w=+1$. The events from the sidebands are asigned a negative weight. We choose sideband regions that are close to the signal region as this ensures that the background in the sidebands is similar to the background beneath the signal region and thus can be used to subtract the background beneath the signal region. Therefore events in the sidebands are a assigned a weight $w=-\frac{\text { sumofeventsinsignalregion }}{\text { sumofeventsinsidebands }}$. Therefore if a kinematic variable is weighted by this weight variable, then one obtains distributions for which only the signal events are present while effectively subtracting the background contribution. This method ensures that a large fraction of background events are subtracted from our analysis while keeping as many signal events as possible. This method of background subtraction using event weights has an advantage that it can be used when performing an event based analysis.

\subsubsection{Nucleon Resonances}

The Radphi experiment was devised to select $\omega$ events that were produced diffrac- 


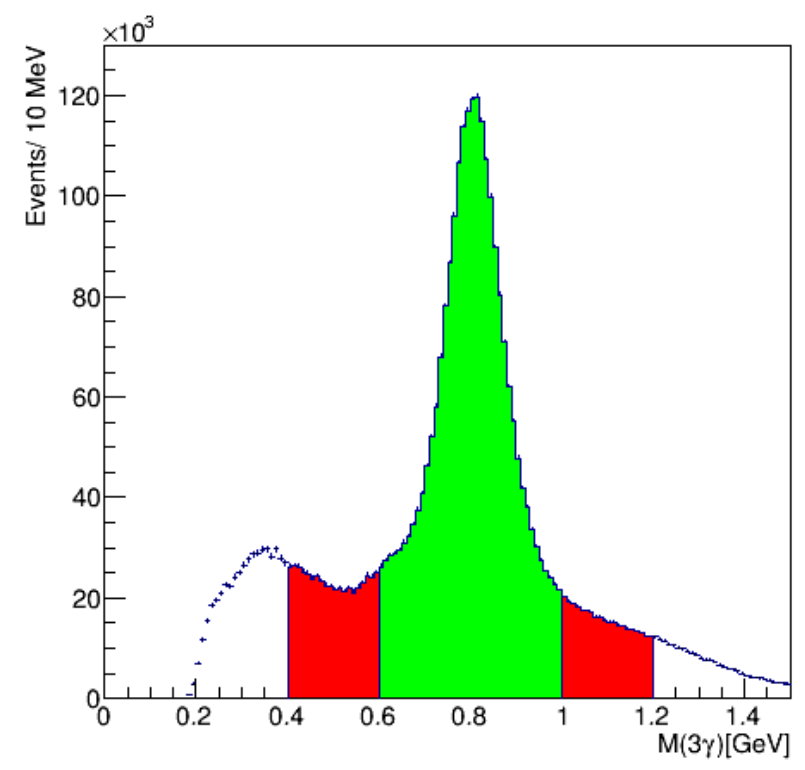

Figure 4.3.1: $3 \gamma$ invariant mass showing sideband(green) and signal(red) regions

tively, that is events with a recoil proton in the final state. However reactions with an excited baryon in the final state had a non negligible acceptance and the $\omega$ signal seen in the invariant mass was the same irregardless of which baryon produced it. Since in this analysis we are interested in $\omega_{\text {s }}$ that were produced diffractively, events with a proton resonance in the final state had to be effectively suppressed. One way of suppressing these events was to apply cut on the total energy that was transferred to the recoiling nucleon. A $500 \mathrm{MeV}$ cut on the total energy of the forward system with respect to the incident photon was applied.This cut reduced the acceptance of $\omega$ reconstruction from $\Delta$ reactions by a factor of 0.6. However, this cut did not completely eliminate contribution from nucleon resonances. To estimate the resonance contribution after performing the energy cut, the azimuthal angle $\Delta \phi$ between the recoil plane and the scattering 
plane was used.The recoil plane is defined by the incident beam direction and the direction of the recoiling nucleon, while the scattering plane is defined by the incident beam and the $\omega$ momentum vector.It is expected that in the case of diffractive scattering, where the recoiling nucleon is not excited all momenta of the particles lie in the same plane.Therefore significant tails outside the diffractive peak in the $\Delta \phi$ distribution can be interpreted as contributions from proton resonances.

4.3.2shows $\mathrm{MC} \Delta \phi$ distributions from, $\gamma p \rightarrow \omega p$ and $\gamma p \rightarrow N^{*}$ reactions. The distributions reveal a difference in shape between the diffractive and resonance production. the real data distribution is fitted to a sum of the MC shapes shown in left panel 4.32 and the fit revealed a net $23 \%$ contribution form diffractive events.Although in this analysis we assume only $\Delta$ production,this estimate inlcudes contributions from higher $N^{*}$ resonances. To subtract the contribution of these $N^{*}$ events, the method of sideband-subtraction described above was employed. For events in the signal region a positive weight was assigned, w = +1 , was assigned while for events in the side-band regions a negative weight was assigned, $\mathrm{w}=-\frac{\text { sumofbackgroundeventsinsignalregion }}{\text { sumofeventsinsidebandregions }}$. To subtract the contribution from background and non-diffractive events, the product of the weights from invariant mass and deltaphi sideband subtraction was applied to the events in the analysis. This method of weights ensures the possibility of performing an event based interpretation analysis.

In this analysis, the final state for $\gamma p \rightarrow \omega p$ reaction is identified by one charged 


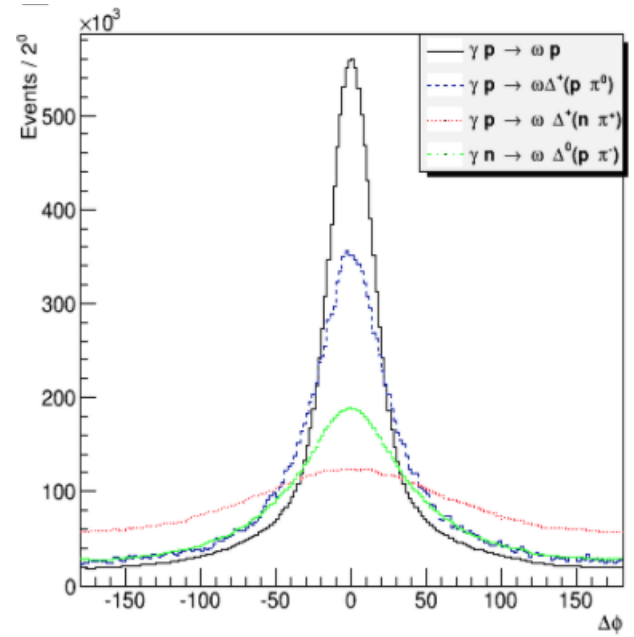

(a)

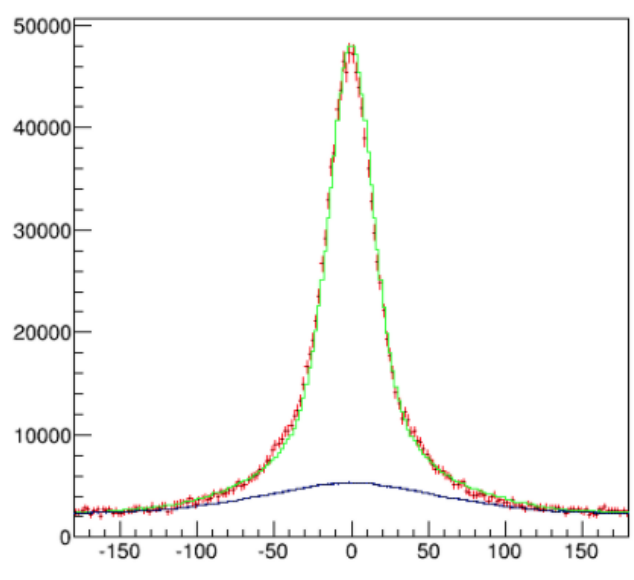

(b)

Figure 4.3.2: Left panel shows the $\Delta \phi$ distributions from different MC reconstructed reactions while the right panel shows the distribution from the real data $3 \gamma$ sample. The distribution on the right is fitted to a sum of the four MC distributions shown on the left. The fit is represented by the green curve while the resonance contribution is represented by the blue curve.

particle (recoil proton) and 3 neutral particles $(\gamma \mathrm{s})$. The effect of competing reactions, the experimental data $|\mathrm{t}|$ distribution is plotted . Left panel ?? shows the $|\mathrm{t}|$ distribution of experimentally measured events before sideband subtraction and right panel after sideband subtraction. Both distribution are fitted with an exponential function $\mathrm{Ae}^{b t}$ in the range 0.3 -1.0 $\mathrm{GeV}^{2}$. The change in slope is an indication of effective supression of competing reactions(background)

\subsection{MonteCarlo and Acceptance}

Inorder to understand the response of the detector subsystems and also the effect of kinematic cuts on the final states, the Radphi experiment used Monte Carlo simulations. These Monte Carlo simulations were done for two different reasons. 


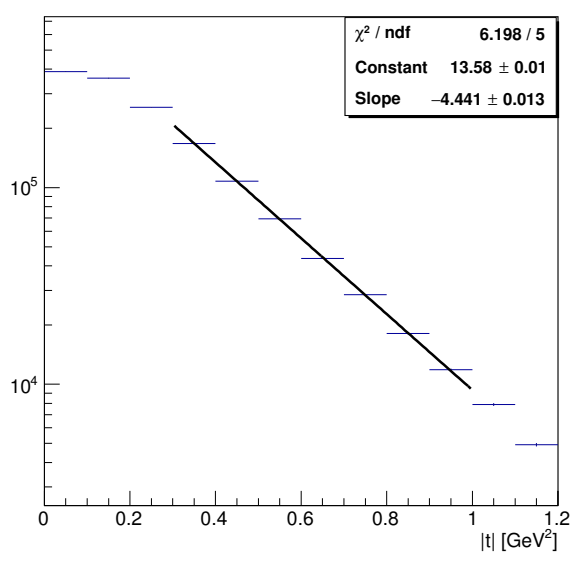

(a)

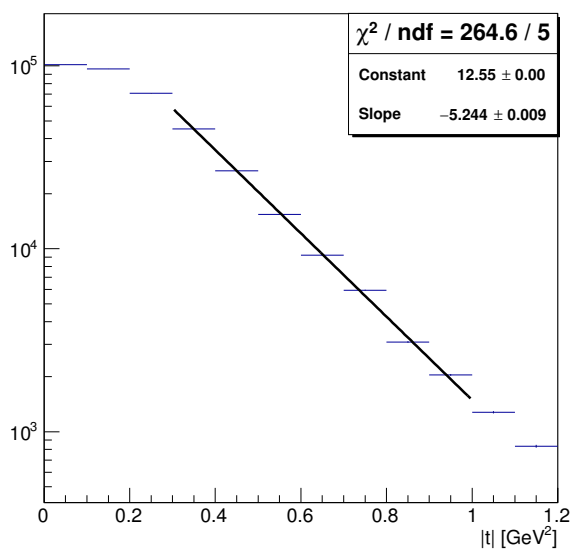

(b)

Figure 4.3.3: Left panel shows the $|\mathrm{t}|$ distribution from $3 \gamma$ sample before sideband subtraction. The distribution on the right is obtained after the sideband subtraction method has been applied.

(i) To study electromagnetic and hadronic background inorder to refine data selection and enhance physics signals.

(ii) Estimate the probability that an event of a given kinematics will be kept by the analysis that is detector acceptance.

A Monte Carlo event generator developed for peripheral photoproduction reaction was adapted for the Radphi experiment from a version previously used by E852 experiment. One major addition to E852 code was incorporation of the Fermi motion in the initial state that was needed for correct treatment of a nucleon in a nuclear target. We generated a total of 100 million $\gamma \mathrm{p} \rightarrow \omega\left(\pi^{0} \gamma\right) \mathrm{p}$ events. The photon beam was unpolarized and momentum transferred to the target assumed to obey a standard exponential distribution $e^{b t}$. The generator produced 4 -vectors for the final state particles including Breit-Wigner width of the $\omega$ in 
the generation. The recoil proton mmentum was generated considering a proton in the nuclear target as quasi-free nucleon that did not interact with the residual $A_{1}$ nuclear system [Mihaljo thesis ] After generation the events were processed by Gradphi, a GEANT3 [3]] simulation of the Radphi detector .GEANT3 is a detector simulation tool developed at CERN. Graphi propagated each of the simulated particle tracks from the event vertex through the Radphi detector, taking into account geometry and other specifics of detector components resulting in a simulated set of detector signals.During the tracking, the energy loss of each particle caused by interactions of particles with materials in Radphi detector. The Gradphi output was then processed by the same Radphi reconstruction software that was used to process experimental data and the same selection criteria used to select experimental data events was used to select the simulated events.

\subsection{Measurement Formalism}

The cross section for a reaction is proportional to the probability of the reaction happening and is found by determining the yield of reaction products under well defined geometrical conditions for known incident flux of particles. A cross section has the dimensions of an area, with the most commonly used unit is the barn where 1 barn $=10^{-28} \mathrm{~m}^{2}$. For the Radphi experiment, only the differential cross section rather than total cross section were measured directly due to the fact that the detectors cover only a small region of space. Therefore to get the total cross section, the differential cross section is integrated over the final state 
kinematic variables The differential cross section for the $\gamma p \rightarrow \omega p$, in this analysis is extracted using the equation:

$$
\frac{d \sigma}{d X_{i}}=\frac{N_{\text {data }}}{L A \Delta X_{i} R}
$$

where $X_{i}$ are the kinematic variables, $\mathrm{A}$ is the detector acceptance and $\mathrm{R}$ is the decay rate into the final state taking into account of branching ratios of the intermediate states, $X_{i}$ Are the kinematic variables, $N_{\text {data }}$ are the number of experimental signal events measured in the kinematic range $\Delta X_{i}$, $\mathrm{L}$ is the integrated luminosity which depends on the beam flux, $\Phi_{\gamma}$, total live time of the run, $t_{\text {live }}$ and target thickness.

$$
L=\Phi_{\gamma} t_{\text {live }} \Delta_{B e}
$$

\section{Kinematic Variables}

The kinematic variables a differential cross section can be binned in in the case of a two body final state $(\mathrm{p} \omega)$ are limited to one independent variable through energy and momentum conservation and the invariance of the final state's azimuthal angle. Thus the kinematic variable can be choosen to be either a function of the polar angle of the final state particle or the lorentz invariant mandelstam variable t. For this analysis the kinematic variable of choice is the lorentz invariant mandelstam variable, $|\mathrm{t}|$. Classically, the magnitude of momentum transferred to to the target particle is a measure of the collison volence, a large momentum 


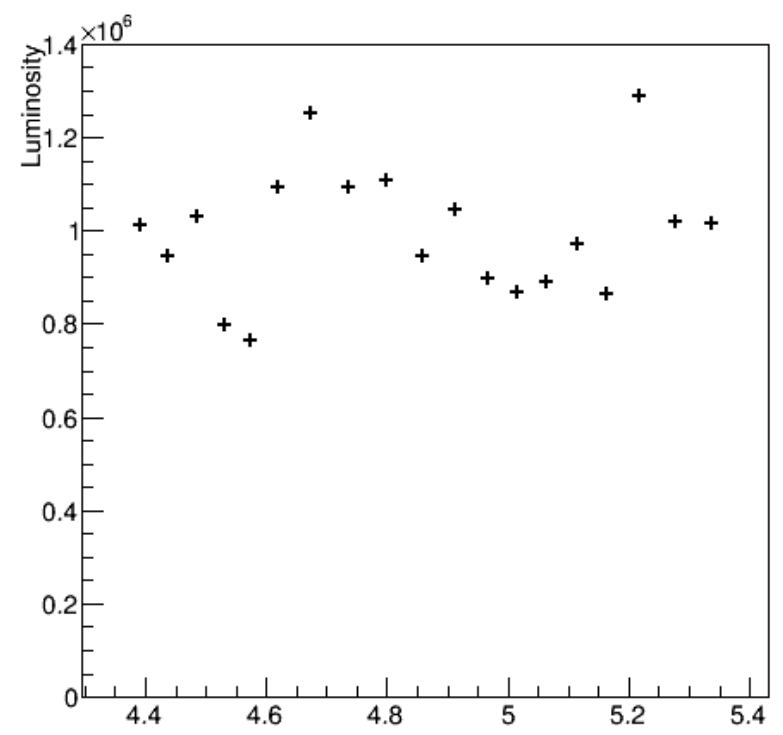

Figure 4.5.1: Integrated luminosity. The integrated luminosity for each tagger channel plotted as a function of incident energy. This factor was used in $\gamma p \rightarrow \omega p$ analysis. The $\mathrm{y}$-axis is in units of $\mu^{-1} \mathrm{~b}$

transfer is considered a violent blow to the target particle.

There are 12 bins in the range $\left(0.0 \mathrm{GeV}^{2} \leqslant|\mathrm{t}| \leqslant 1.2 \mathrm{GeV}^{2}\right)$ with a binwidth of $0.1 \mathrm{GeV}^{2}$. The bin limits were set based on the resolution of the -t distribution.

\subsubsection{Acceptance Calculation}

The acceptance calculation arises from comparing the number of reconstructed(passed through the simulated detector) simulated events to the number of generated simulated events.That is :

$$
\text { Acceptance }=\frac{\text { Reconstructedevents (withcuts })}{\text { Generatedevents }}
$$

Other experimental acceptance factors that were not accounted for by the simulation but are directly measured are 
(i) BSD pixel efficiency: The probability that a charged particle going through all the 3 BSD layers will result in a triple coincidence known as pixel, between overlapping counters in the 3-layers. To determine the pixel efficiency two factors need to be considered.

(a) The electronics and efficiency of light collection

(b) Recoil loss in one of the BSD layers, that is particles recoiling at lower poalr angles had more barrel material obstructing their path and thus less probability to pass through all the BSD layers than particles recoiling at $90^{\circ}$ with the same energy. This factor was taken into account in the Monte Carlo acceptance and had to be taken out when pixel efficiency is measured using data.

The pixel efficiency was computed as the product of efficiencies of the 3 individual layers. Thus the product of individual layer efficiency gave a total BSD pixel efficiency correction actor of 0.7

(ii) CPV efficiency: THIS actor accounted for hits in the CPV that fell inside the veto window but were not associated with the trigger. The CPV had a total rate of approximately $75 \mathrm{MHz}$. This rate combined with the veto time window of $6 \mathrm{~ns}$ would lead to an accidental fraction of 0.68 assuming that all hits in a CPV plane are uncorrelated.

These efficiency factors were included in the calculation of the total cross section.

\subsubsection{Normalization}


The integrated luminosity for the experiment is the product of the total tagger count with the tagging efficiency and the target thickness. The total tagger count was monitored by scalers on the individual tagging counters. Their sum was corrected for dead time using the ratio of gated and ungated reference scalers. The tagging efficiency is defined as the number of tagged photons incident on the target divided by the sum of the tagger scalers. As such, it is a product of a geometric factor and an electronic factor. The geometric factor is the number of tagged photons reaching the target per hit in the tagger focal plane. The electronic factor takes into account the fact that the tagger scalers count somewhat faster than the rate of electrons hitting the focal plane. This arises from double-firing discriminators and electrons that produce signals in more than one focal plane counter. The electronic factor is measured by analyzing the tagger time spectra on a run-byrun basis. The geometric factor is calculated from the bremsstrahlung differential cross section, with corrections for beam-target misalignment, which will be described later. The total integrated luminosity for the experiment obtained in this way was $18.9 \pm 0.4 \mathrm{pb}^{-1}$. To estimate the acceptance of the on-line trigger and the off-line analysis, a MC simulation was employed. Events were generated assuming $\gamma p \rightarrow \omega p$ kinematics. The initial-state momentum of the target proton was smeared according to a spherically symmetric nuclear momentum distribution described by the sum of two Gaussians. The widths of the two Gaussians and their relative weights were adjusted to make the out-of-plane recoil proton momentum distribution match the real data. The scattering plane is defined by 


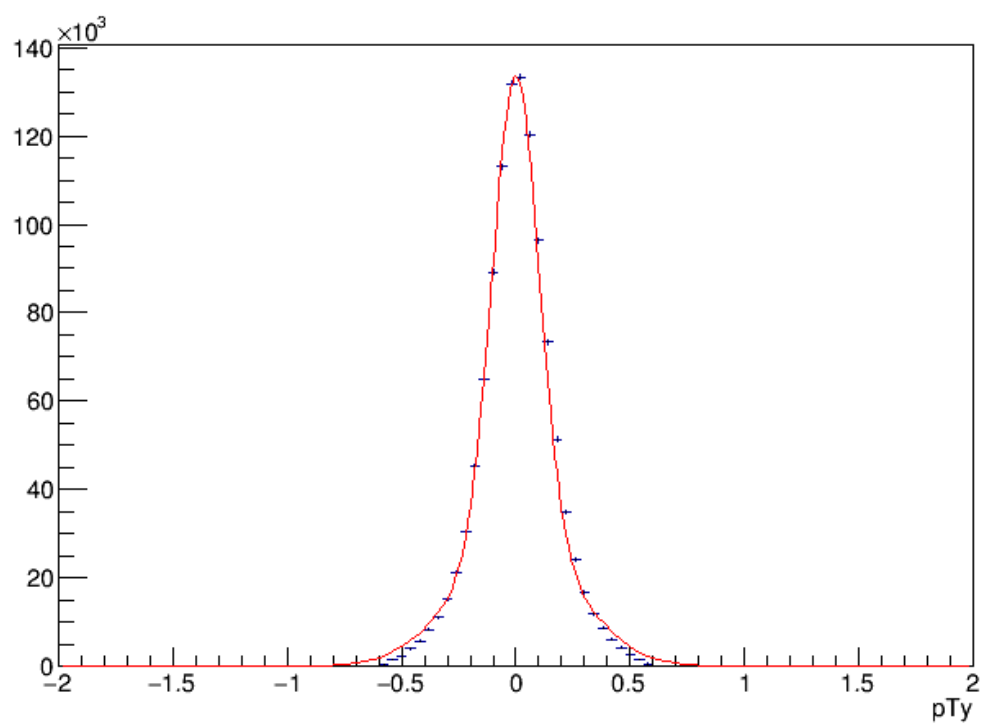

Figure 4.5.2: Real data out-of-plane momentum distribution for the $3 \gamma$ sample. The MC distribution is plotted as a red curve

the incident beam direction and the total momentum of the $3 \gamma$ system in the final state. For a free nucleon target the recoil proton momentum is constrained to lie in this plane by momentum conservation, but in the case of a nuclear target, the Fermi motion of the initial-state proton in the nucleus results in an out-of-plane component. The parameters that describe the nuclear momentum distribution are given in 4.5 .5 .

A spectator model in which the outgoing proton does not interact with the residual A-1 nucleon system in the final state was used to generate the recoil proton momentum. The $\omega$ was generated with zero net polarization and decayed into $\pi^{0} \gamma$ with a branching ratio of $8.28 \%$. The angular distribution of the in the centerof-mass frame of the reaction was specified by an exponential t-distribution. The generated t-slope was adjusted to agree with the experimental data for the range 
Table 4.5.5: The widths $\left(\sigma_{1}\right.$ and $\left.\sigma_{2}\right)$ and relative heights $\left(f_{1}\right.$ and $\left.f_{2}\right)$ of the two Gaussians that describe the out-of-plane recoil proton momentum distribution seen in the real data, compared with that seen in reconstructed MC. In the MC events the out-of-plane recoil proton momentum distribution is controlled by the initial state nuclear momentum distribution described by a sum of two Gaussians parametrized as shown in the last row. The parameters of that distribution were adjusted to obtain a good agreement between the reconstructed MC and the real data. Given errors are statistical only.

\begin{tabular}{lllll}
\hline & $\begin{array}{l}\sigma_{1} \\
{[\mathrm{MeV}]}\end{array}$ & $\mathrm{f}_{1}$ & $\begin{array}{l}\sigma_{2} \\
{[\mathrm{MeV}]}\end{array}$ & $\mathrm{f}_{2}$ \\
\hline data & $99 \pm 6.0$ & $0.53 \pm 0.01$ & $226.7 \pm 4.0$ & $0.47 \pm 0.02$ \\
MC & & & \\
reconstructed & $101 \pm 1.0$ & $0.72 \pm 0.01$ & $256 \pm 1.0$ & $0.28 \pm 0.01$ \\
MC generated & 90 & 0.86 & 450 & 0.14 \\
\hline
\end{tabular}

$0.3<|\mathrm{t}|<1.0 \mathrm{GeV}^{2}$, as described below in 4.44 .5 .4 left panel, shows the $\mathrm{t}-$ distribution for the $3 \gamma \omega$ sample, after sideband subtraction around the $\omega$ peak to suppress the background under the $\omega$ peak. In the $|t|$ range of $(0.3,1.0) \mathrm{Ae}^{b t}$ with the slope parameter $\mathrm{b}=5.4 \mathrm{GeV}^{-2}$. The same slope in $\mathrm{MC}$ reconstructed data is obtained using $\mathrm{b}=5.5 \mathrm{GeV}^{-2}$ in the $\mathrm{MC}$ generator. The acceptance function for the reaction $\gamma p \rightarrow \omega\left(\pi^{0} \gamma\right) p$ based upon a sample of $100 \mathrm{M}$ generated events, is shown in the right panel of Figure 4.5.4. Errors shown in the plot are statistical only. Figure 4.5.5shows the mean and RMS of the reconstructed -t as function of generated -t.

The average MC acceptance of the detector was obtained by dividing the total number of reconstructed $\omega$ events by the number generated. Its value is listed in Table 4.5.6, together with its systematic error. The error includes uncertainties arising from the choice of various tunable parameters in the MC generator and 
in the detector response model used by the simulation.

The beam-target misalignment is estimated by analyzing the azimuthal distribution of the scattering plane. This distribution for $3 \gamma$ events with invariant mass in a range $0.7<\mathrm{M}(3 \gamma)<0.9 \mathrm{GeV} / \mathrm{c}^{2}$ is shown in the left panel of Figure 4.5.3. Any shift of the beam from the geometric center of the target is expected to produce an azimuthal asymmetry because of the varying amount of material seen by the recoil proton as it exits the target. To quantify this effect the beam-target displacement was varied in the simulation and the azimuthal asymmetry compared with that seen in real data. The final distributions are shown in Figure 4.5.3, where the MC distribution is obtained by shifting the beam by $5 \mathrm{~mm}$ in the $+\mathrm{x}$ direction. The distributions are fitted with the function

$$
Y(\phi)=A+B \sin (\phi+C)
$$

with $\mathrm{A}, \mathrm{B}$, and $\mathrm{C}$ being fitting parameters. The fits are shown by solid curves in Figure 4.5.3. The flat dashed lines represent the case where the beam spot is centered on the target. The deviation from a sinusoidal shape seen in the real data between $75^{\circ}$ and $135^{\circ}$ is related to the non-uniform efficiency of a few BSD counters in that range. A beam shift of $5 \mathrm{~mm}$ with respect to the target

\subsection{Summary}

In this chapter, we attempted to separate exclusive $\omega$ signal events from background and suppress reactions with an excited nucleon in the final state by as- 


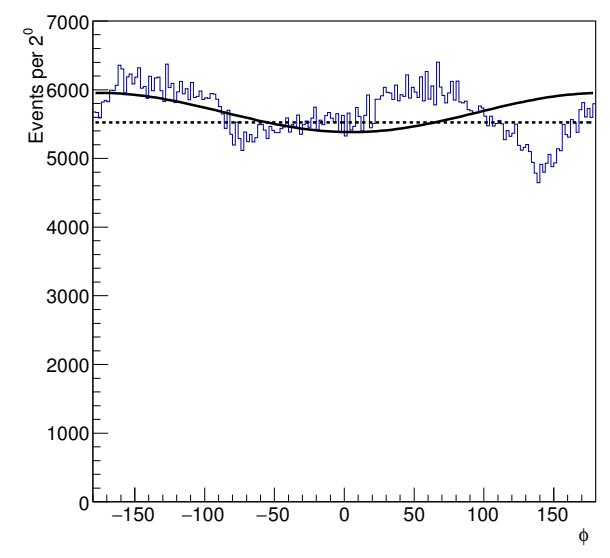

(a)

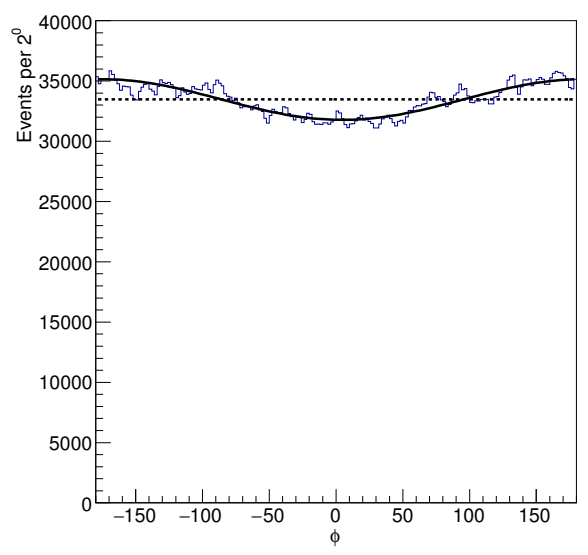

(b)

Figure 4.5.3: Azimuth distribution of the transverse momentum of the forward $3 \gamma$ system after sideband subtraction in invariant mass and $\delta \phi$ from MC (left)

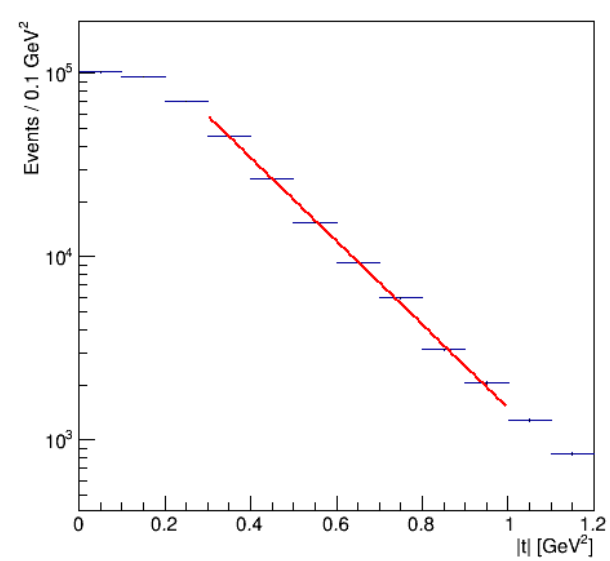

(a)

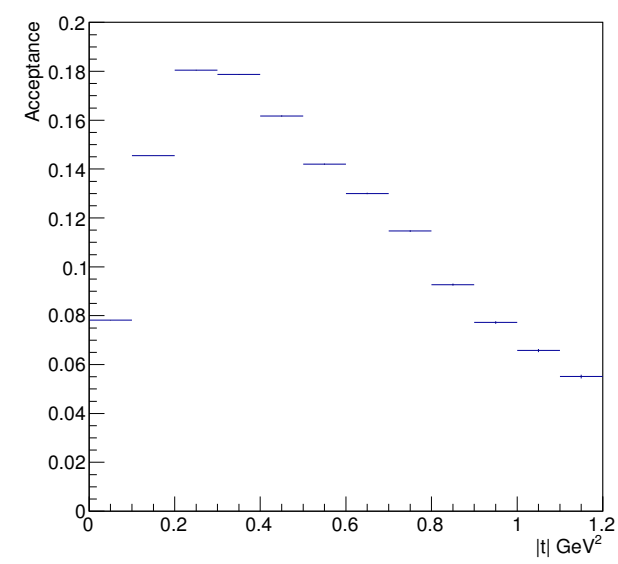

(b)

Figure 4.5.4: The t-distribution from the $3 \gamma \omega$ sample (left). sideband subtraction in invariant mass has been used to form this plot. MC-acceptance (right) obtained from the ratio of the reconstructed and generated -t distributions with a slope parameter, b, of $5.5 \mathrm{GeV}^{2}$

signing event weight using the method of sideband subtraction. By weighting each event with a weighting factor obtained from sideband subtraction we obtain an approximation of the signal distribution.In the next section we estimate the 


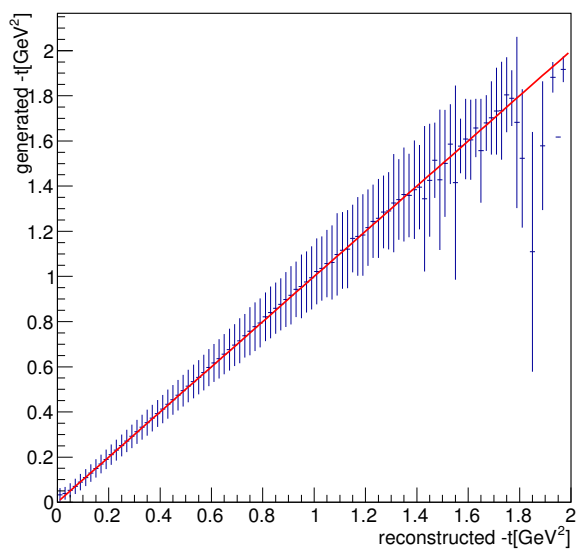

(a)

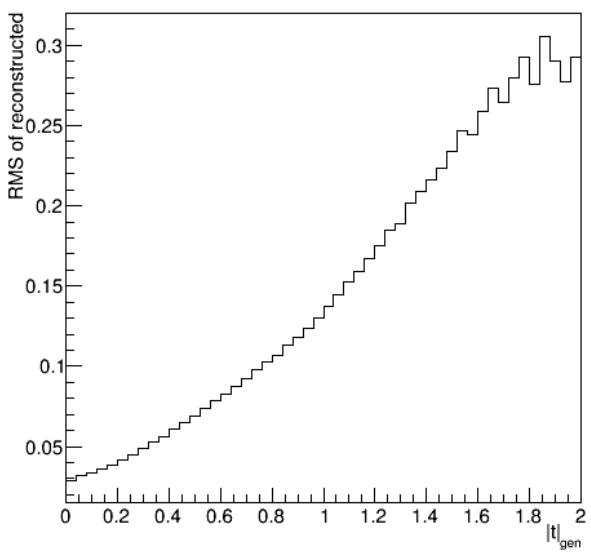

(b)

Figure 4.5.5: The mean and RMS of the reconstructed $\mathrm{t}$ as function of generated t obtained with the MC simulation. The left plot shows the centroid and width in the form of error bars, while the right plot shows the width separately. Results reported here extend up to $|\mathrm{t}|=1.2 \mathrm{GeV}^{2}$.

uncertainty introduced by this signal-background separation. 
Table 4.5.6: The total $\omega$ yield measured from $3 \omega$ sample, cross section normalization factors and total $\omega$ photoproduction cross section. The acceptance assumes an exponential t-distribution with a slope $\mathrm{b}=5.5 \mathrm{GeV}^{-2}$ this cross section includes contribution from $\Delta$ and $\mathrm{N}^{*}$ resonances as well as diffractive $\omega$ p production.

\begin{tabular}{lll}
\hline Quantity & Value \pm Error & Units \\
\hline$\omega$ yield $\left[10^{6}\right]$ & $1.71 \pm 0.09$ & \\
B.R $\left(\omega \rightarrow \pi^{0} \gamma\right)$ & $0.0828 \pm 0.003$ & \\
luminosity & $75.6 \pm 1.5$ & $\mathrm{pb}^{-1}$ \\
acceptance & $0.18 \pm 0.01$ & \\
pixel efficiency & $0.70 \pm 0.03$ & \\
CPV accidentals & $0.68 \pm 0.01$ & \\
$\sigma$ & $3.2 \pm 0.2$ & $\mu \mathrm{b}$ \\
\hline
\end{tabular}




\section{Chapter 5}

\section{Results}

After selecting events with the correct final state reactions, the experimentally measured and simulated events were used to measure the observables. The differential cross sections and spin density matrix elements are presented.The observables will be used in interpretation of production mechanism of vector mesons at Radphi energies. The spin-density matrix elements will be used in intepretation analysis to describe the production mechanism of $\omega$ meson. The differential cross section and unpolarized spin density matrix elements are presented in bins of momentum transfer ,-t, and incident photon energy bins.

\subsection{Spin Density Matrix Elements Extraction}

Before extracting the spin density matrix elements, both the experimental data and Monte Carlo data are binned in momentum transfer -t and incident photon

energy. For $|\mathrm{t}| \leqslant 0.6 \mathrm{GeV}^{2}$ we use bins of width $0.1 \mathrm{GeV}^{2}$ while for $\mathrm{t}>0.6$ $G e V^{2}$ we have 3 wider bins of variable widths. This is because the at large - $\mathrm{t}-$ bins we have fewer signal events and thus making the bins wider ensures we have more statistics to perform a fit. The decay angular distributions in this analysis are measured in both Helicity and Gottfried Jackson frame defined in ??and are 
fitted with Equation 2.3.5 using the extended maximum likelihood method. and the three unpolarized spin density matrix elements are returned as fit parameters.

\subsubsection{Maximum Likelihood Fitting}

To extract the SDMEs the method of maximum likelihood was used The CERNLIB package MINUIT is used to minimize the negative log likelihood,

$$
-\ln \mathscr{L}=\sum_{i=1}^{N_{\text {data }}} w_{i} \ln \left|W\left(\Omega_{i}, \rho^{0}\right)\right|^{2}+\frac{1}{N_{\text {gen }}} \sum_{j=1}^{N_{\text {rec }}}\left|W\left(\Omega_{j}, \rho^{0}\right)\right|^{2}
$$

in each $\left(|\mathrm{t}|, \mathrm{E}_{\gamma}\right)$. several iterations of the fit were done and the iteration with the best likelihood was used to extract the fit parameters. To find the estimators $\hat{\theta}$ for

a set of parameters $\vec{\theta}$ with $\lambda$ detected events. We define the extended likelihood function as

$$
\mathscr{L}=\left(\frac{\lambda^{n}}{n !} e^{-\lambda}\right) \prod_{i}^{n} f\left(\vec{\theta}, X_{i}\right)
$$

where the term in the parenthesis is the poisson probability of observing $n$ events given the expected number $\lambda, X_{i}$ are the event kinematics the second term is the likelihood term where the product is over data events and $f\left(\vec{\theta}, X_{i}\right)$ is the probability density function and in this analysis is defined as

$$
f\left(\vec{\theta}, X_{i}\right)=\frac{W\left(\Omega_{i}, \rho\right.}{\left.\int W \Omega\right) A(\Omega) d \Omega}
$$

where $\mathrm{W}\left(\Omega_{i}, \rho\right)$ is the angular distribution function and is a function of the decay angles and spin density matrix elements and the denominator is defined such that 
the probability density is normalized to 1 . The best estimators for the parameters are obtained by maximizing $\mathscr{L}$. Since it is easier to work with summations than products, the likelihood function is written as the log likelihood and since the natural logarithm is an entirely non decreasing function,the estimators that minimize $-\ln \mathscr{L}$ will maximize $\mathscr{L}$. The $\log$ likelihood is written as.

$$
-\ln \mathscr{L}=-n \ln \lambda+\ln n !+\lambda-\sum_{i}^{n} \ln f\left(\vec{\theta}, X_{i}\right)
$$

the expected number of events $\lambda$ is obtained by

$$
\lambda=\int W(\Omega) A(\Omega) d \Omega
$$

Since we are interested in minimizing the $-\ln \mathscr{L}$ and not its actual value we drop all the terms that do not depend on the parameters $\rho$ from Equation 5.1.4

$$
-\ln \mathscr{L}=-\sum_{i}^{n} \ln W\left(\Omega_{i}, \rho\right)+\lambda
$$

The integral Equation 5.1.5 is done numerically since we do not have analytic expression for the acceptance $\mathrm{A}(\Omega)$, the integral therefore becomes

$$
\lambda=\frac{C}{N_{\text {raw }}} \sum_{i}^{N_{\text {rec }}} W\left(\Omega_{i}, \rho\right) A\left(\Omega_{i}\right)
$$

\subsubsection{Background}

Inorder to separate non- $\omega \rightarrow \pi^{0} \gamma$ events from signal events, each event comes with a weight factor $w_{i}$, this weight factor effectively suppresses the non -signal 
events. To account for this background subtraction in the likelihood, the log likelihood function is rewritten as

$$
-\ln \mathscr{L}=-\sum_{i}^{n} \ln W\left(\Omega_{i}, \rho\right)+\frac{1}{N_{g e n}} \sum_{i}^{N_{\text {rec }}} W\left(\Omega_{i}, \vec{\rho}\right)
$$

The weight factor $\mathrm{w}_{i}$ is applied as an exponent to the single-event likelihood function.In the limit of large $\mathrm{N}$, the effects of the background inside the signal window is statistsically cancelled by the reciprocal weights of events in the sidebands

$$
-\ln \mathscr{L}=-\sum_{i}^{n} w_{i} \ln W\left(\Omega_{i}, \rho\right)+\frac{1}{N_{\text {gen }}} \sum_{i}^{N_{\text {rec }}} w_{i} W\left(\Omega_{i}, \vec{\rho}\right)
$$

where $w_{i}$ is the weight factor used for background subtraction. This method works perfectly for uniform weights. We address in the next section the case where we have non-uniform weights such as for this analysis where the incident beam was a tagged beam and subtraction of accidental coincidences between the detected events and taggers was done using negative weights while the true coincidence events were assigned a positive 1 weight.

\subsubsection{Handling Non-Uniform Weights}

Following the sPlot technique, ?? a correct expression is derived for a likelihood function The modifiedlog-likelihood function that takes into account non-uniform weight factors is defined and ensures that statistical errors from the EML fit are handled carefully.The sPlot method employs negative weights for events in the mass sidebands. Negative weights were also applied to sidebands in $\Delta \phi$ to 
remove the effects of $\mathrm{N}^{*} \omega$ production. The width of the peak at $\Delta \phi=0$ changes somewhat as a function of -t Eigure 5.1.2, so the negative $\Delta \phi$ weight factor needed to be adjusted independently for each t-bin to achieve the proper subtraction. By contrast, the $3 \omega$ lineshape of the $\omega$ resonance did not show significant variation with tEigure 5.1.1, so the mass sidebands were fixed for all $\mathrm{t}$ bins. The product of these two weights and the tagging accidentals subtraction weight formed the weight factor $\mathrm{w}_{i}$ used to weight each reconstructed decay event i. Since in this analysis, the weight factors are not simply the event weight factors but a related quantity which is 2-dimensional and is computed based on the weight factors. The modified Likelihood function

$$
-\ln \mathscr{L}=\lambda-\sum_{i}^{n} w_{i} R(\Omega) \ln \left(W\left(\Omega_{i}, \rho\right)\right)
$$

where $\mathscr{L}$ is the modified Likelihood function, and $\lambda$ is defined as

$$
\lambda=\int R(\Omega) W(\Omega) A(\Omega) d \Omega
$$

where $R(\Omega)$, derived from data alone, is defined as,

$$
R(\Omega)=\frac{\langle w\rangle}{\left\langle w^{2}\right\rangle}
$$

The numerator and denominator in equationEquation 5.1.3 are obtained by forming 2-D histograms with weight $\mathrm{w}$ and $\mathrm{w}^{2}$. 

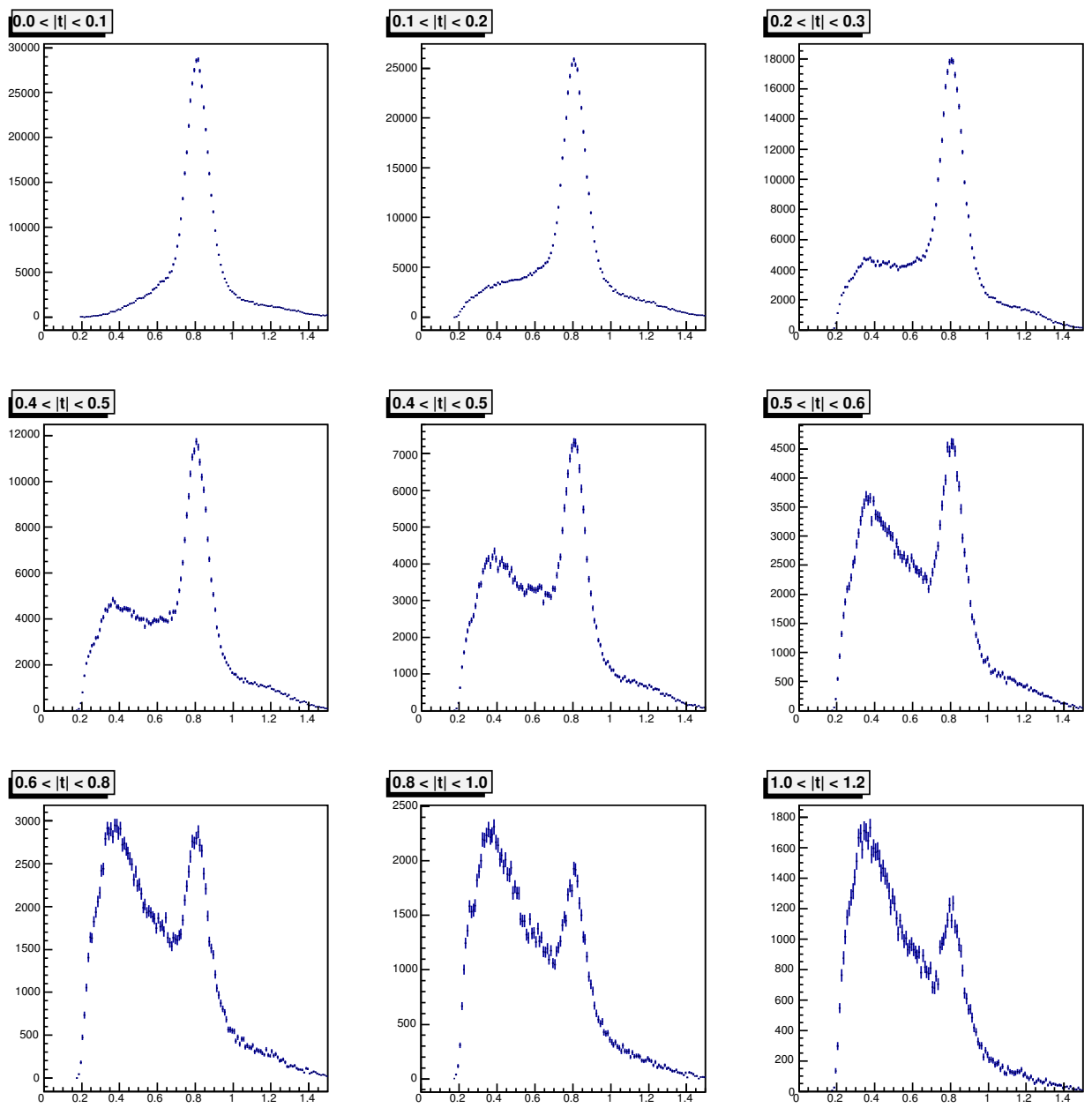

Figure 5.1.1: $3 \gamma$ invariant mass distributions for selected t-bins. Although the background grows as $|\mathrm{t}|$ increases, the lineshape does not vary with $|\mathrm{t}|$. 

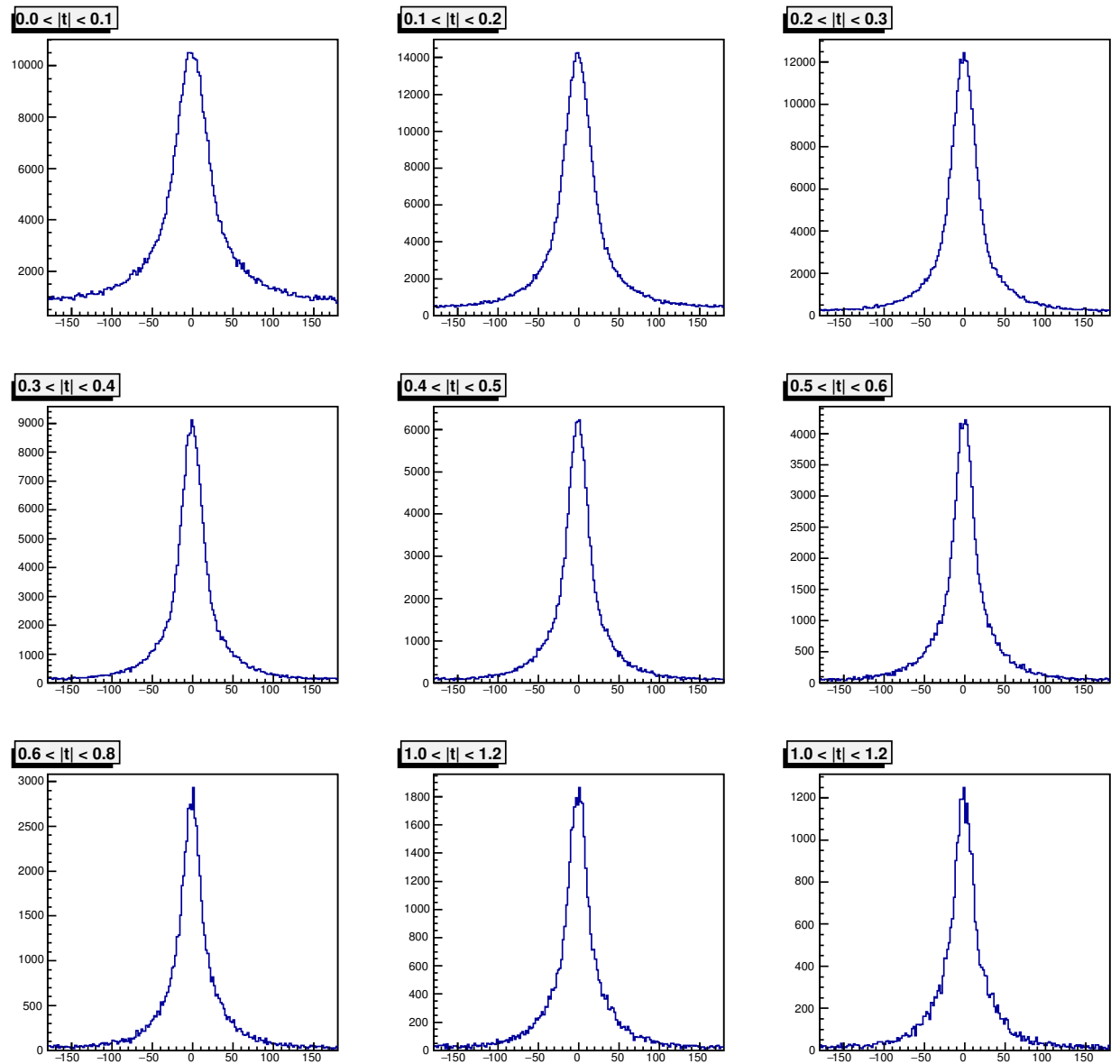

Figure 5.1.2: $\Delta \phi$ distribution for selected t-bins. These distributions show changing $\Delta \phi=0$ peak width as a function of $|\mathrm{t}|$ 


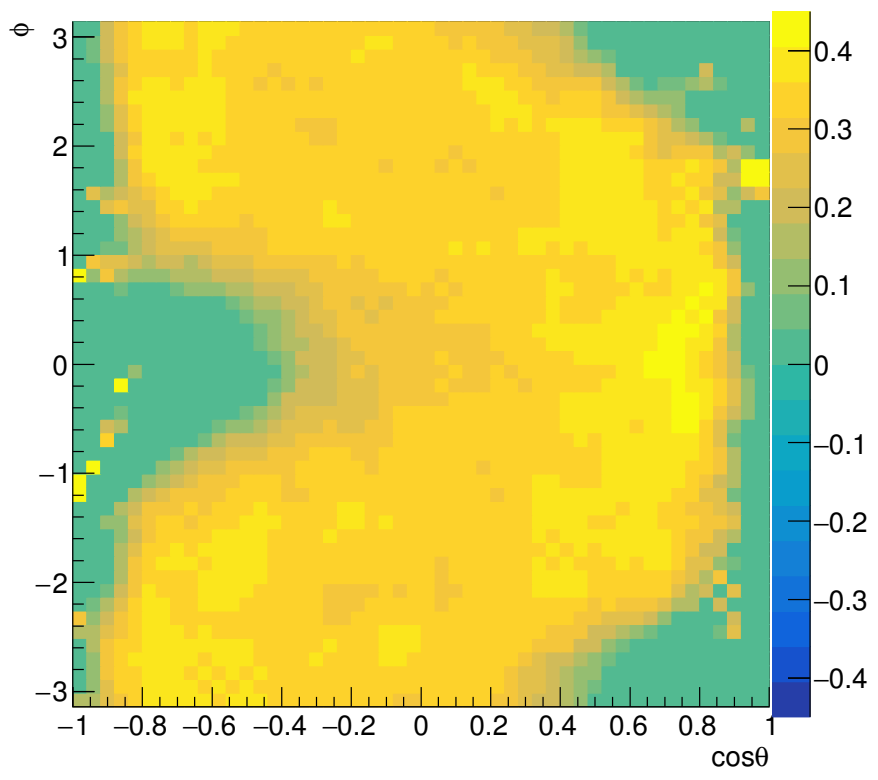

Figure 5.1.3: R-function used for maximum likelihood fitting for weighted events for a selected t-bin.To form this plot, events with acceptance below $30 \%$ have been eliminated

With this likelihood function defined we then proceed to perform the fit and extract the SDMEs. Before performing the fit, regions in which our MonteCarlo acceptance had significant tails but there was no real data had to be etched out. Failure to do this, leads the fit to regions where our acceptance was not trusted and this led to results which were not a reflection of our angular distributions. One way of catching this effect was to look at the $\mathrm{W}$ function obtained after fitting a tbin,if nodes are seen in regions where the acceptance is small this implies that the W function is failing to reproduce the acceptance corrected angular distribution. Left panel Figure 5.1.4 shows this effect while the right panel shows the $\cos \theta_{H}$ projection. Figure 5.1.5 (left panel) shows the $\mathrm{W}$ function obtained after 2D 


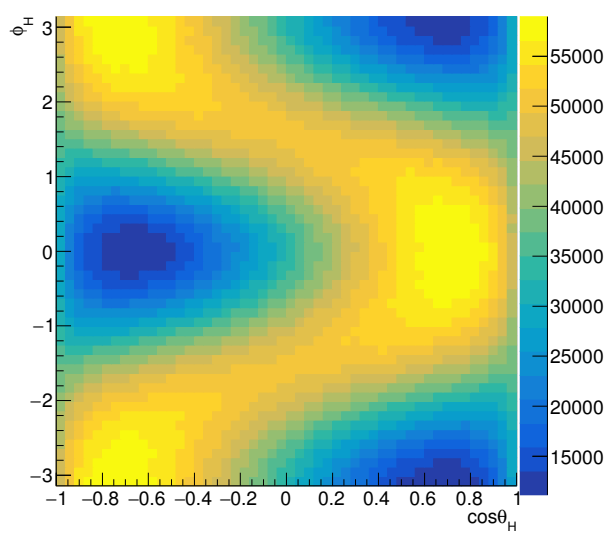

(a)

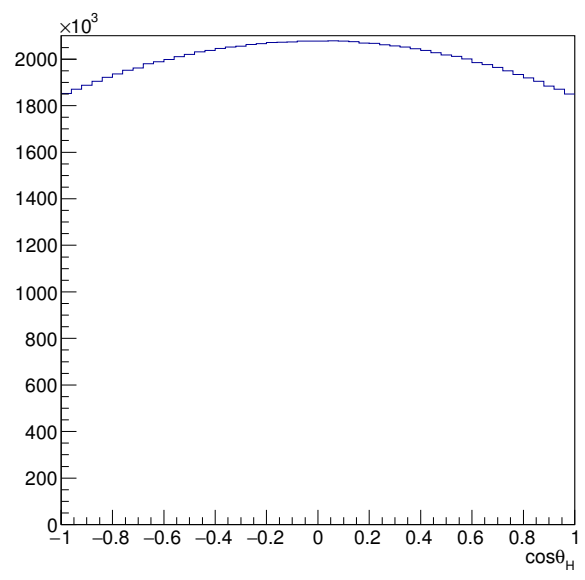

(b)

Figure 5.1.4: Fit function Equation 2.3.4 returned by the fit plotted as a 2D histogram (left) panel for a selected t-bin.This plot is obtained after fitting an angular distribution that has not been etched The right panel shows the $\cos \theta_{H}$ projection.

regions of the angular distribution where the acceptance could not be trusted were etched out and events in those regions excluded from the fit. Events in the region where the acceptance is expected to be good are then fitted to ?? and the SDMEs extracted.

Figure 5.1.4 and Figure 5.1.5 shows the importance of this etching out regions in which the acceptance is not reliable. If these regions are not etched out, The fit function fails to reproduce the shape seen in Figure 5.2.1 and will lead to the physics amplitude showing nodes in regions where the acceptance is small. The results presented in this thesis are obtained after this etching process has been performed to the angular distribution. To ensure that the fit is working, the $\mathrm{W}$ function is checked to ensure that no nodes are present in regions where we do not have reliable acceptance. This procedure was done for both Helicity and 


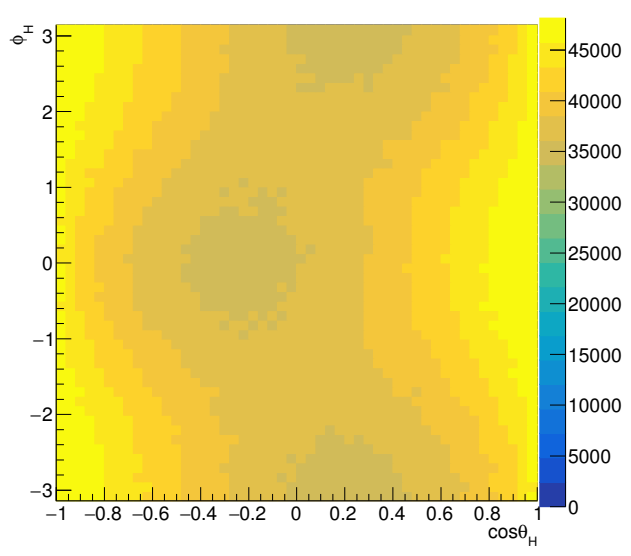

(a)

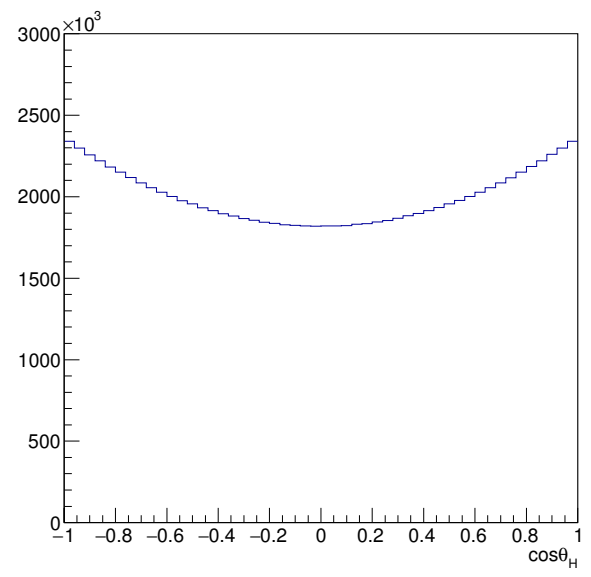

(b)

Figure 5.1.5: Fit function Equation 2.3.4 returned by the fit over the data that has been etched to remove regions where there is no experimental data (left panel). The right panel shows the $\cos \theta_{H}$ projection.

Gottfried Jackson frame angular distributions.

\subsection{Angular Distributions}

The SDMEs were extracted in the helicity and Gottfried Jackson frames [40]. These two frames are connected by a rotation about the y-axis. The data were binned in momentum transfer t-bins. Angular distributions in each bin were subjected to an unbinned EML to Equation 2.3.5. The width of each t-bin was dictated by the resolution with which $\mathrm{t}$ can be reconstructed. Figure 4.5.5 shows the reconstruction resolution of $\mathrm{t}$ from reconstructed Monte Carlo. There are a total of 9 t-bins in the range $|\mathrm{t}|=(0.0-1.3) \mathrm{GeV}^{2}$. The first 7 bins with $|\mathrm{t}|<$ $0.6 \mathrm{GeV}^{2}$ have a bin width of $0.1 \mathrm{GeV}^{2}$, while for $|\mathrm{t}|>0.6 \mathrm{GeV}^{2}$ the bin width was increased to compensate for the dropping statistics at large $|\mathrm{t}|$. 

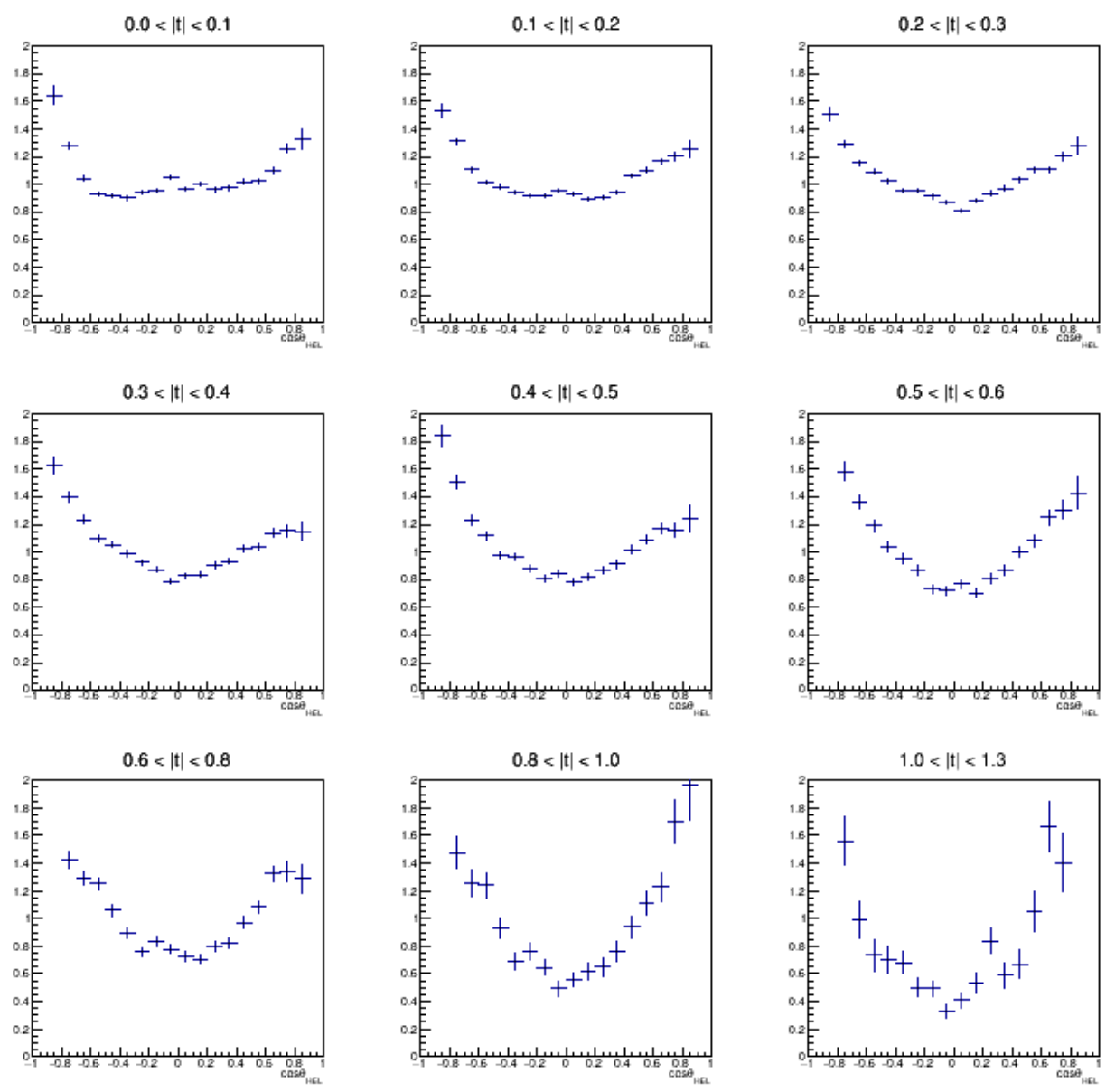

Figure 5.2.1: Acceptance-corrected helicity frame $\cos \theta_{H}$ distributions for selected t-bins, obtained by taking the ratio of the experimentally measured $\cos \theta_{H}$ and Monte Carlo reconstructed $\cos \theta_{H}$ distributions. The scale of the $\mathrm{y}$ axis is arbitrary. These plots show how the $\cos \theta_{H}$ shape varies over the full range in $\mathrm{t}$ for the experiment. The errors displayed are statistical. 

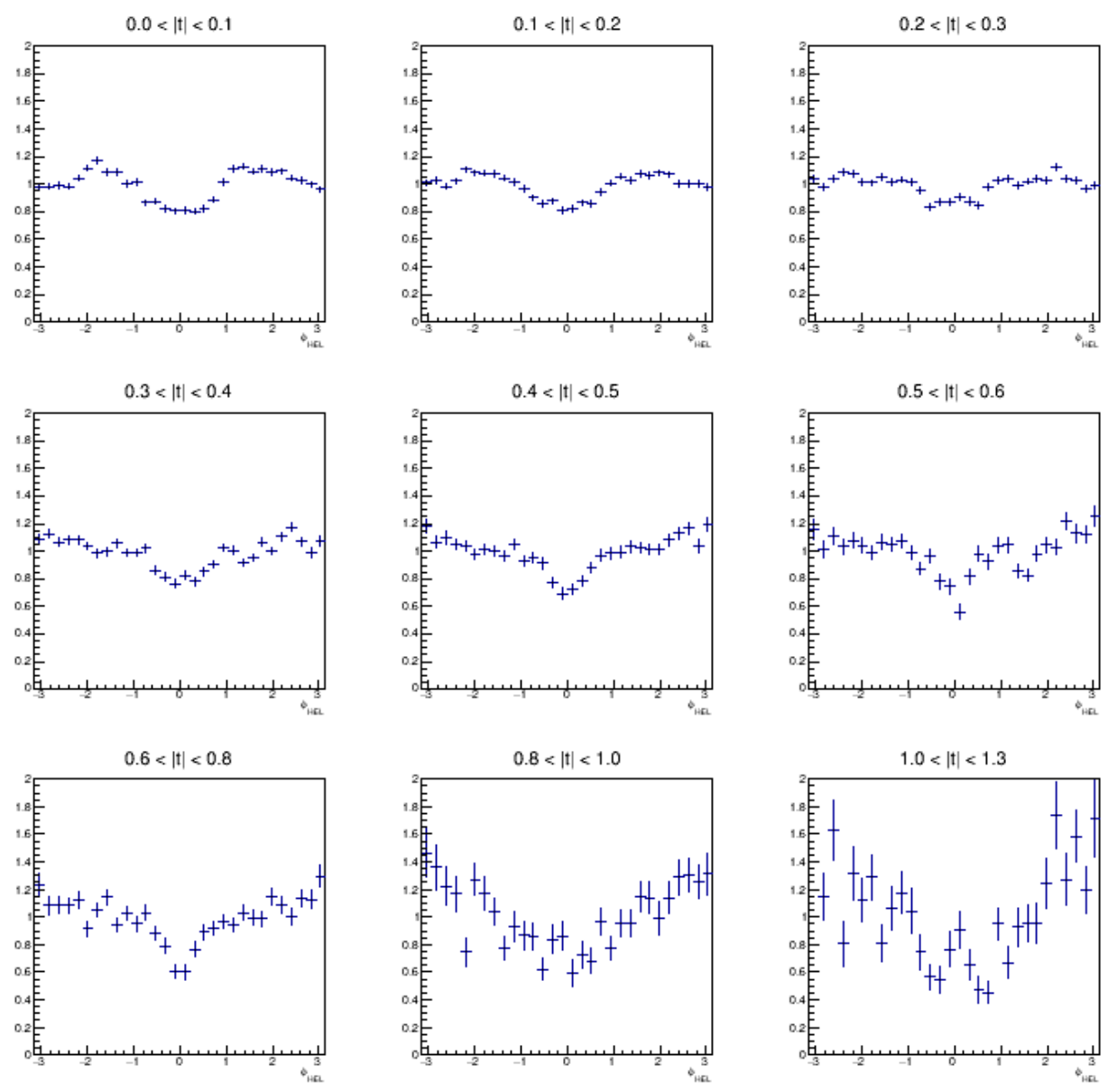

Figure 5.2.2: Acceptance-corrected helicity frame $\phi_{H}$ distribution for selected tbins, obtained by taking the ratio of the experimentally measured $\phi_{H}$ and Monte Carlo reconstructed $\phi_{H}$ distributions. The scale of the y axis is arbitrary. These plots show how the $\phi_{H}$ shape varies over the full range in t for the experiment. The errors displayed are statistical. 

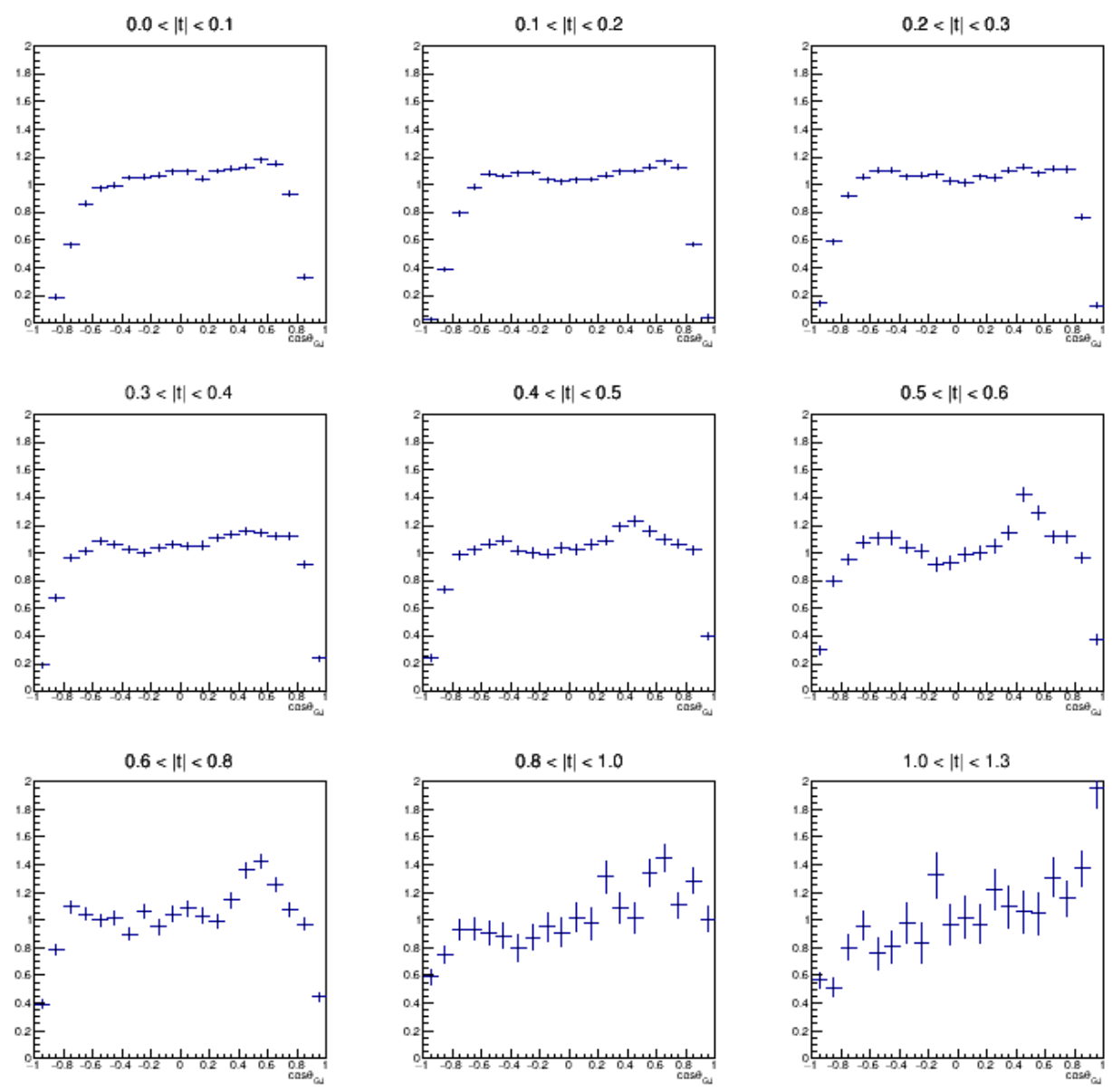

Figure 5.2.3: Acceptance-corrected Gottfried Jackson frame $\cos \theta_{G J}$ distribution for selected $|\mathrm{t}|$ bins, obtained by taking the ratio of the experimentally measured $\cos \theta_{G J}$ and Monte Carlo reconstructed $\cos \theta_{G J}$ distributions. The scale of the $\mathrm{y}$ axis is arbitrary. These plots show how the $\cos \theta_{G J}$ shape varies over the full range in $t$ for the experiment. The errors displayed are statistical 

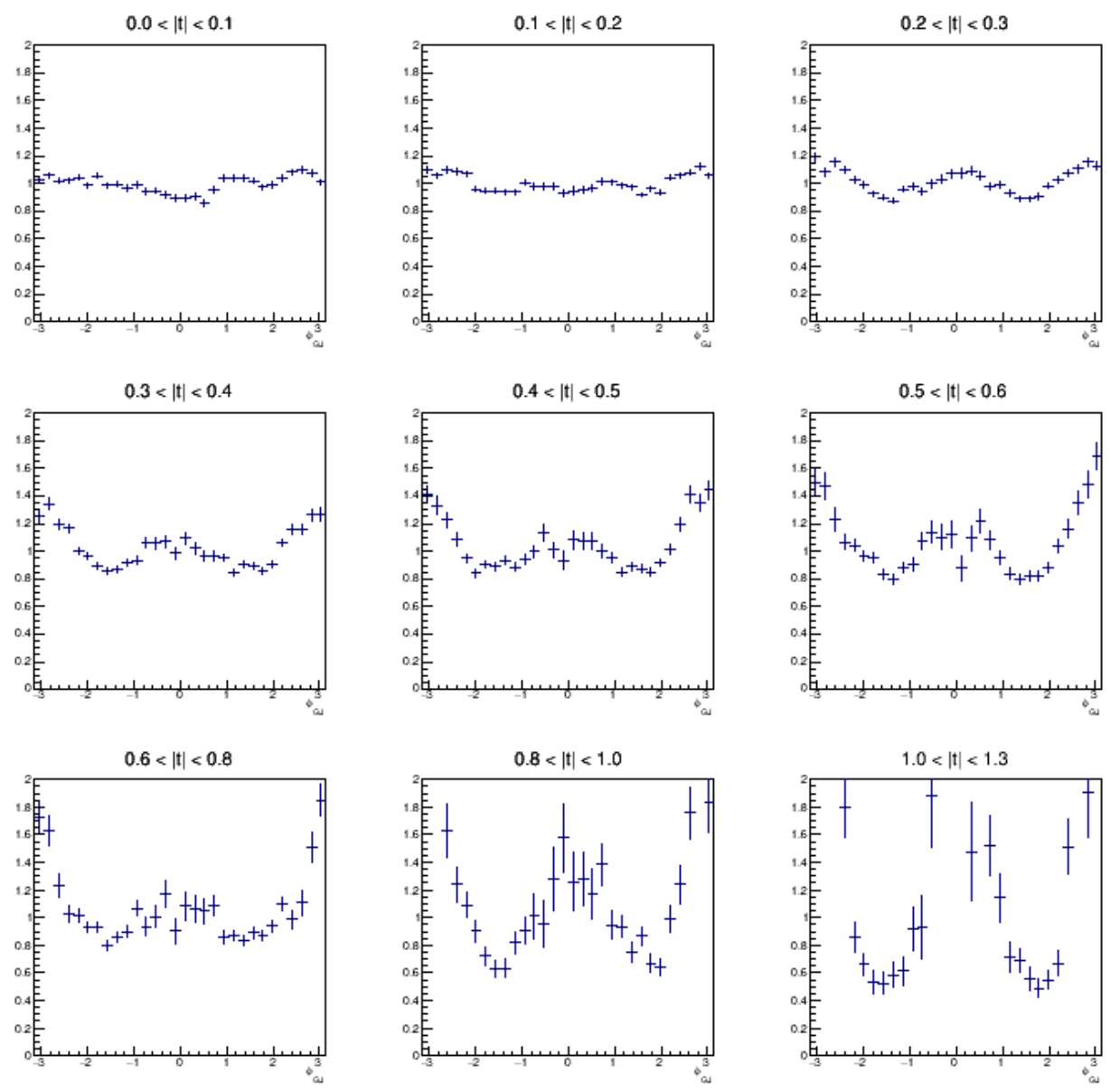

Figure 5.2.4: Acceptance-corrected Gottfried Jackson frame $\phi_{G J}$ distribution for selected $|\mathrm{t}|$ bins, obtained by taking ratio of the experimentally measured $\phi_{G J}$ and Monte Carlo reconstructed $\phi_{G J}$ distributions. These plots show how the $\phi_{G J}$ shape varies over the full range in $t$ for the experiment. The errors displayed are statistical 
In both Gottfried Jackson (GJ) and Helicity frames, if the angular distribution is flat in $\phi$ and a $(1+\cos 2 \theta)$ shape is seen, this implies that the spin of the $\omega$ meson is aligned along the z-axis. In the helicity frame, this condition is described as s-channel helicity conservation, consistent with the assumption that the $\omega$ meson was produced diffractively. Similarly, alignment of the $\omega$ spin with the $\mathrm{z}$ axis in the GJ frame implies the dominance of the exchange of a spin-0 particle in the t-channel. Figure 5.2.1- Figure 5.2.4 show the acceptance corrected $\cos \theta$ and $\phi$ decay angular distributions for both frames in 9 t-bins.To do the acceptance correction for each t-bin,the real data distribution is divided by the MC phase space distribution for that t-bin. Although we do not fit directly to these distributions, these distributions reflect the underlying physical process.

\section{Goodness of Fit Test}

For maximum likelihood fits, there is no standard method of ensuring that the fits provide an excellent description of the data. However, one can perform qualitative analysis by comparing the real data angular distributions with MC weighted by the fit function with the best fit parameters. As a first step of checking if the fit results are reliable, the $\mathrm{W}(\cos \theta, \phi)$ returned by the fit had to be free of nodes as shown in Figure 5.1.5. The 1D projection $(\cos \theta)$ distribution looks similar to what we observe in our acceptance corrected $\cos \theta_{H}$ helicty frame angular distribution. Next, the real data distributions were compared to MC phase space distributions weighted by the fit function with the nbest fit parameters ?? shows the helicty frame real data distribution after suppressing events with acceptance 

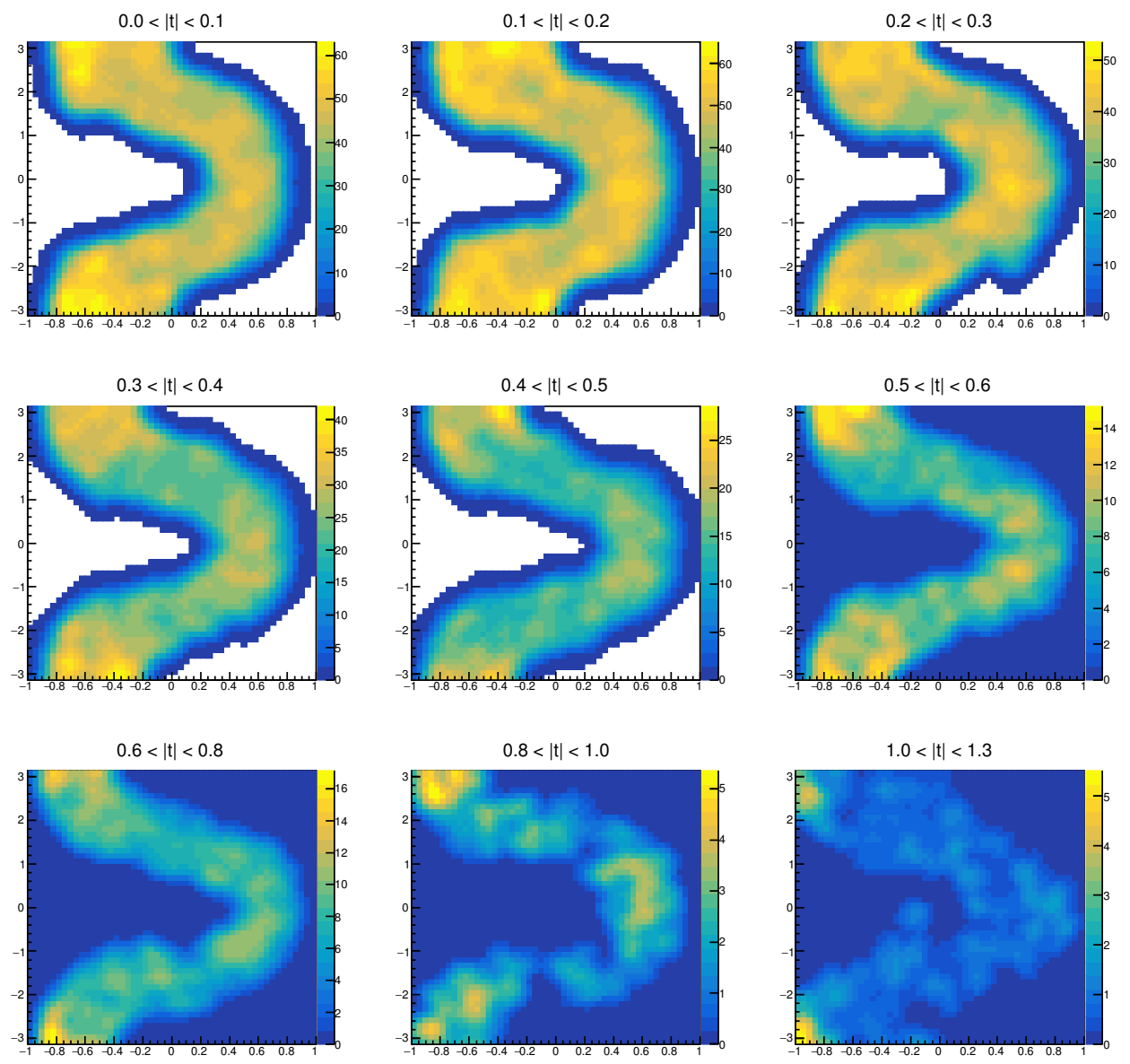

Figure 5.2.5: Experimental data $\phi_{H}$ versus $\cos \theta_{H}$ from the $\omega$ decay in t-bins for the enrgy range (4.4-5.4) GeV. Each $2 \mathrm{D}$ distribution is labelled by the t-bin in which it was obtained.

below 0.3 and etching out regions where we have unreliable acceptance. These distributions are compared with MC phase distributions weighted by the results of the fit Figure 5.2.6. the agreement is good.Both the real data and MC weighted distributions have been smoothed.

We also look at the Gottfried Jackson distributions and compare the real data distributions with the MC phase space distributions weighted by the fit function 


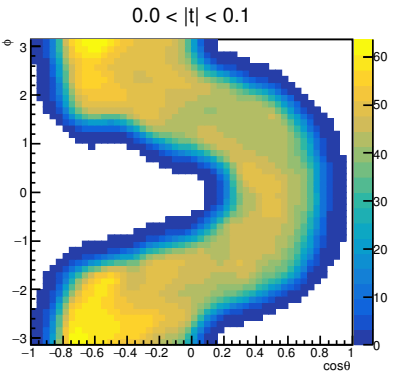

$0.3<|\mathrm{t}|<0.4$

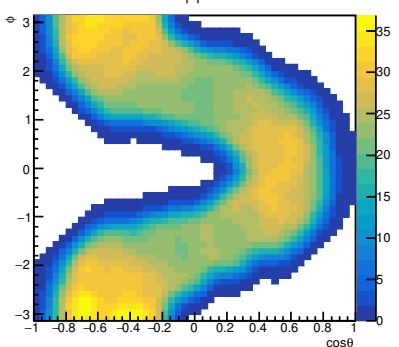

$0.6<|\mathrm{t}|<0.8$

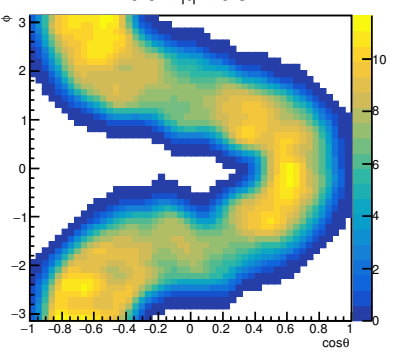

$0.1<|t|<0.2$

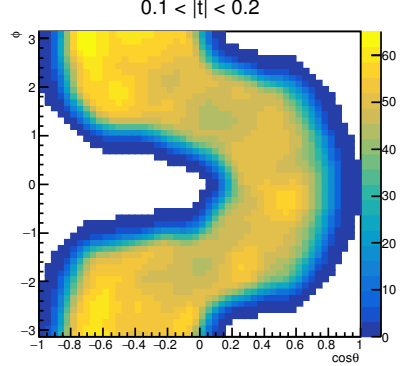

$0.4<|\mathrm{t}|<0.5$

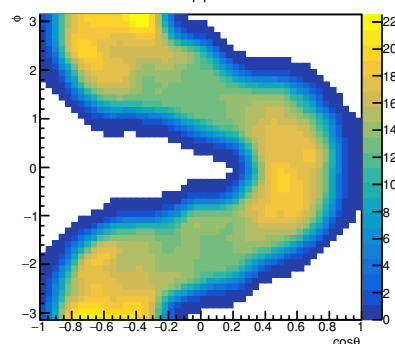

$0.8<|t|<1.0$

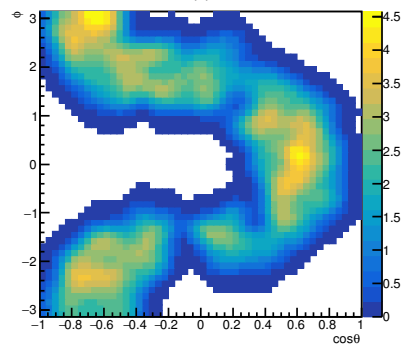

$0.2<|t|<0.3$

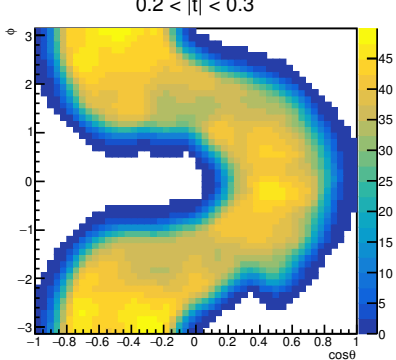

$0.5<|\mathrm{t}|<0.6$

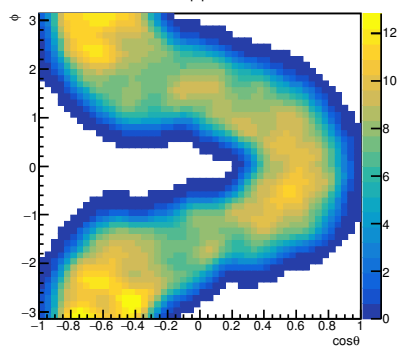

$1.0<|t|<1.3$

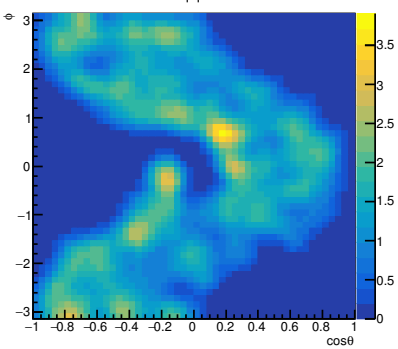

Figure 5.2.6: Helicity frame Monte Carlo $\phi_{H}$ versus $\cos \theta_{H}$ weighted by the results of the fit model. The agreement between Figure 5.2.5 and weighted Monte Carlo is good 

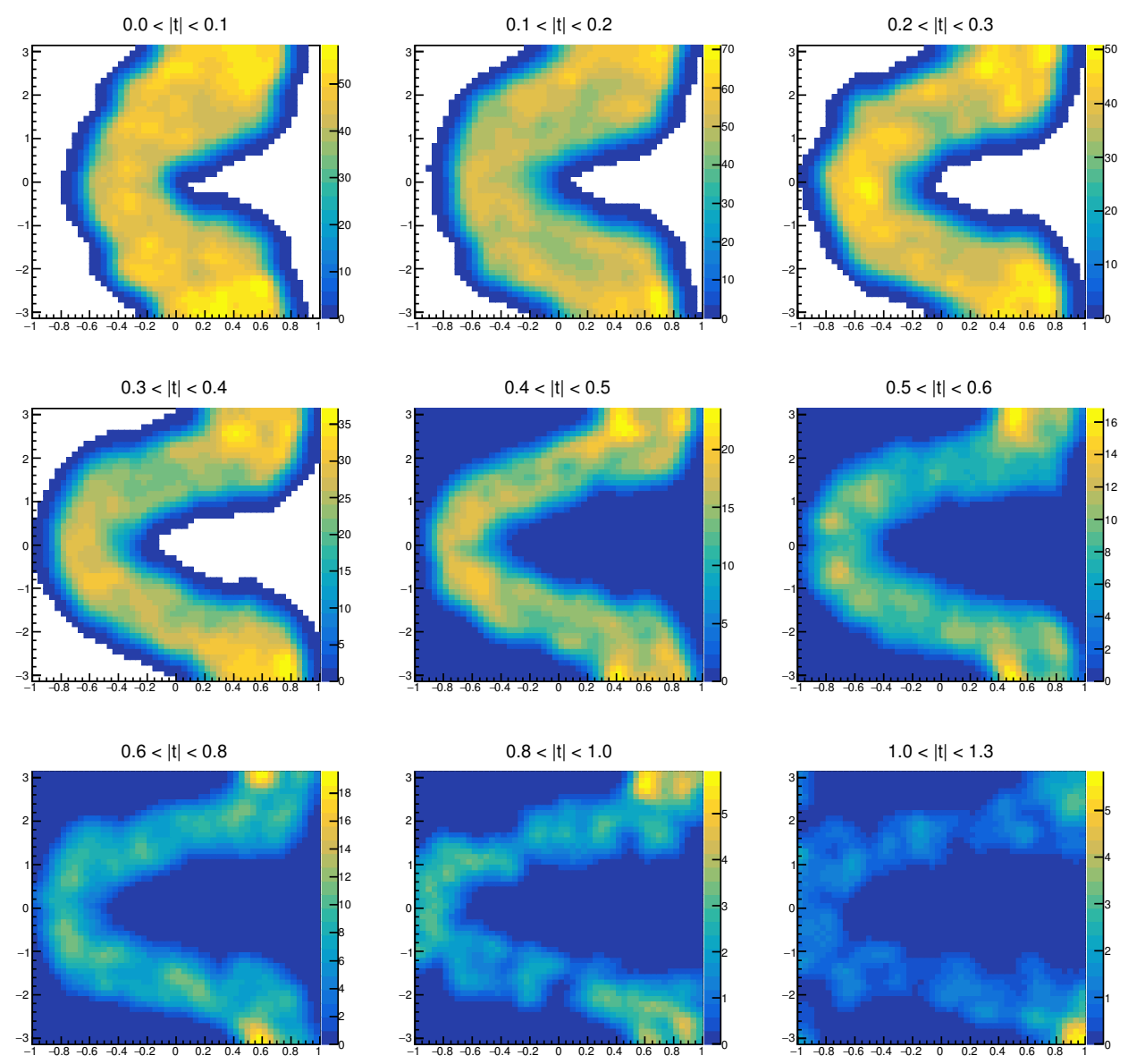

Figure 5.2.7: Experimental data $\phi_{G J}$ versus $\cos \theta_{G J}$ from the $\omega$ decay in t-bins for the enrgy range (4.4-5.4) GeV. Each $2 \mathrm{D}$ distribution is labelled by the t-bin in which it was obtained.

with the best fit parameters. Figure 5.2.7 - Figure 5.2.8 shows the comparison between the real data and $\mathrm{MC}$ phase space weighted by fit function, the agreement between these two distributions is good and the fit function reproduces most of the features seen in real data.

\subsection{Measurement Uncertainity}

Any experimental result, when reported, is usually accompanied by an estimated 

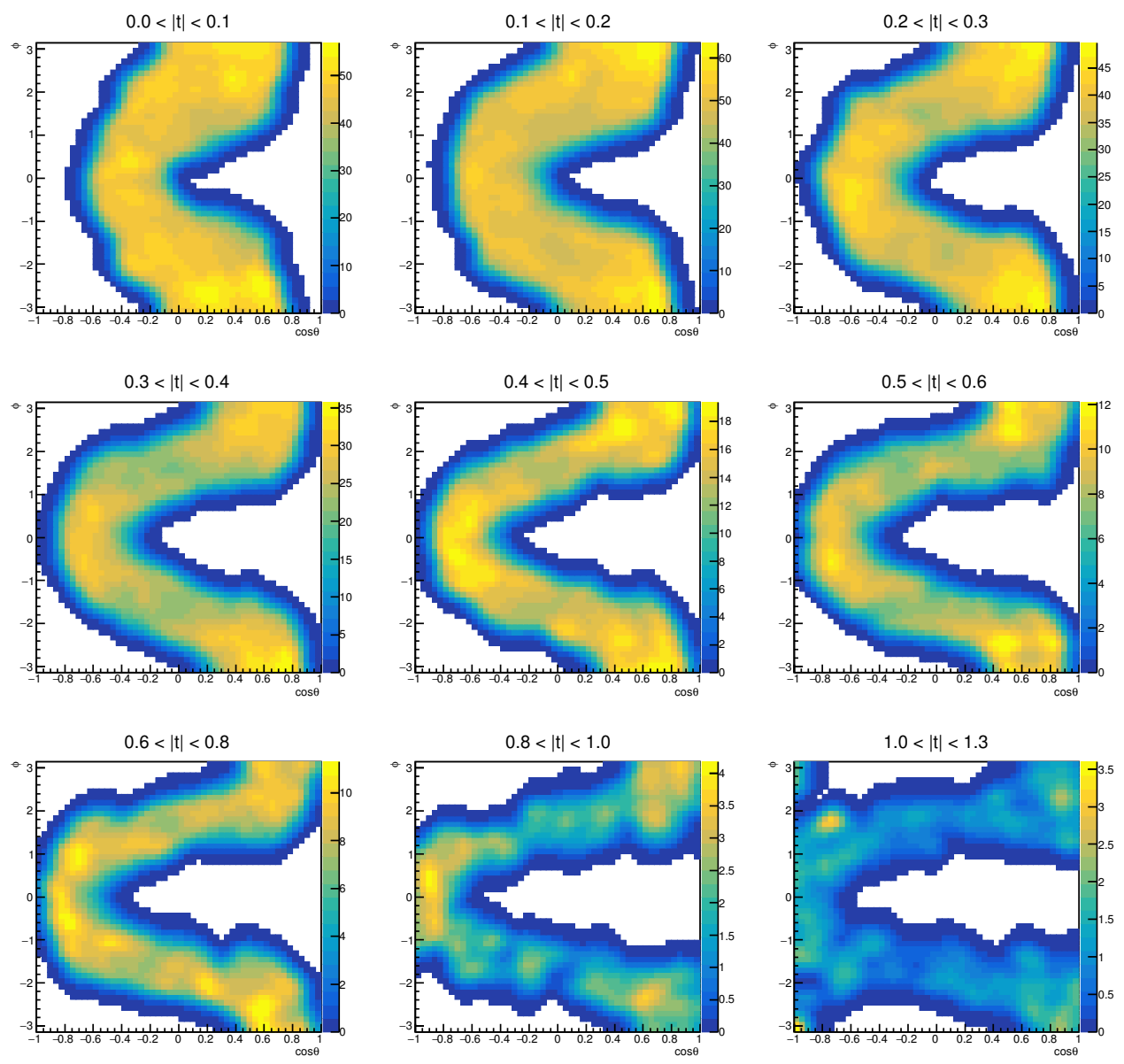

Figure 5.2.8: gottfried Jackson frame Monte Carlo $\phi_{G J}$ versus $\cos \theta_{G J}$ weighted by the result of the the fit model. The agreement between Figure 5.2.7 and weighted Monte Carlo is good 
uncertainty. These uncertainties can be grouped into two categories depending on the way in which the uncertainty value is obtained

(i) Statistical Uncertainty: These are measurement errors due to statistical fluctuations in the data.

(ii) Systematic Uncertainties: These are any other measurement errors other than direct statistics

Statistical and systematic uncertainties are reported as separate values, Individual errors from different sources are summed in quadratures and then reported as a final value. This section deals with uncertainties in the differential cross sections, the cross section being an absolute measurement requires careful study

of the uncertainties. These uncertainties will also be used for the spin density matrix elements.

\subsubsection{Statistical Uncertainty}

Statistical errors in this analysis come from purely the number of events that pass the selection cuts.Since in this analysis each event is associated with a weight factor, statistical error is calculated as

$$
\sigma^{2}=\sum_{i} w_{i}^{2}
$$

Where $\sigma$ is the uncertainty and $\mathrm{i}$ is the index of the number of events considered in forming the result reported and $\mathrm{w}_{i}$ is the weight factor.

\subsubsection{Systematic Uncertainty}


Most of the systematic errors come from procedures used in this analysis. These uncertainties estimate the effect of each choice of selection criteria has on the final result and thus is a measure of how accurate our measurements are.

\section{Invariant Mass Sideband Subtraction Systematic Uncertainty.}

The method of sideband subtraction was used to select kinematic variable for which a pure omega is present. To achieve this it is important to eliminate the the background underneath the signal peak. In this analysis the signal window is chosen to be (0.7-0.9) GeV. A wider cut here would potentially increase the signal to background ratio and also enable us to better understand the signal peak.In this section the SDMEs are extracted using a wider cut for the omega signal window, $(0.675-0.925) \mathrm{GeV}$, to see if this causes any systematic shift. This new

cut causes the number of signal events to increase. The difference between the SDMEs extracted using these two different cuts is used to estimate the systematic uncertainity due to the sideband subtraction method.

\section{Delta-phi sideband subtraction.}

Like wise we can vary the delta phi signal window cut ( used to eliminate proton resonance) to see if this cause any shift in the extracted SDMEs .Since our delta phi distributions are t-dependant, the delta phi signal window was different in each t-bin. The delta phi distribution was wider in the lower t-bins and as $\mathrm{t}$ increases the distribution gets narrower. Therefore the delta phi signal window widths were t-dependant. Although the delta phi distributions have different widths, the signal window was increased by the same amount, $10^{\circ}$, to form the 
alternate windows that were used to estimate the systematic uncertainty. To decide how much uncertainity is created by choosing a different signal window, the SDMEs were remeasured using a different choice of delta phi signal window for both data and MC simulations and the difference between the matrix elements extracted using the nominal signal window and the alternate window were use to estimate the systematic uncertainty introduced by this sideband.

\section{Pion mass Window Systematic Uncertainty}

To select events with 0 kinematics, and reduce contributions from 2 events that leak into our sample, a cut was applied that required the invariant mass of the lightest pair of photons to fall within the pion mass. The invariant mass of the two lightest showers from the $3 \gamma$ sample was required to be to be (0.1-0.18) GeV. This cut was varied by increasing the pion invariant mass window by $55 \mathrm{MeV}$ for both real data and MC simulation. We then remeasure the SDMEs and then evaluate the difference between the SDMEs obtained from this alternate window and the nominal window and estimate the systematic shift that is introduced by varying the pion invariant mass window.

\section{Minimum Total Shower Energy Cut Systematic Uncertainty}

The minimum total shower energy cut was used to refine the online level 3 trigger requirement. To estimate the systematic shift that would arise by varying this minimum energy requirement, We investigate if increasing the minimum total shower energy by $0.3 \mathrm{GeV}$ would cause any systematic shift in the extracted SDMEs. The new minimum total shower energy threshold was set at $3.3 \mathrm{Gev}$, that 
is events with total shower energy greater than $3.3 \mathrm{GeV}$ were kept for further analysis. The SDMEs were remeasured using this new energy threshold requirement and systematic uncertainity estimated.

\section{Acceptance Threshold cut Systematic Uncertainty}

Since we perform an unbinned weighted maximum likelihoodfit to multidimensional data and the average behavior of the weights over the fit domainis defined by a multidimensional function. In this analysis the weight function is defined using the contents of a 2Dimensional histogram of $\phi$ vs $\cos \theta$ Thus the the acceptance threshold is defined as the minmum value of Monte Carlo acceptance as a fraction of the maximum value over the fit doman. The weigh function is overwritten with zero for values below the acceptance threshold. The spin density matrix and differential cross section results are obtained using an acceptance threshold of 0.3. To calculate the systematic shift introducted by setting this threshold, the acceptance threshold is set to 0.1 and the spin density matrix elements remeasured.

\section{Combining the Errors}

All the systematic errors discussed here are added in quadrature to find the total systematic uncertainity for ach t-bin. This is shown as a grey band in Figure 5.4.2

\subsection{Cross Section}

The differential cross sections $\frac{d \sigma}{d t}$ and unpolarized spin density matrix elements for $\omega$ meson photoproduction on a Beryllium target at rest in the region of 4.39 
$\mathrm{GeV}<\mathrm{E}_{\gamma}<5.39 \mathrm{GeV}$ and $9 \mathrm{t}$-bins in the range $0.0<|\mathrm{t}|<1.3 \mathrm{GeV}^{2}$ were measured. The real parts of the spin density matrix elements for the reaction ${ }^{9} \mathrm{Be}(\gamma, \omega p)[\mathrm{A}=8]$ were determined by the method described in section 2.3

\subsubsection{Total and differential cross section}

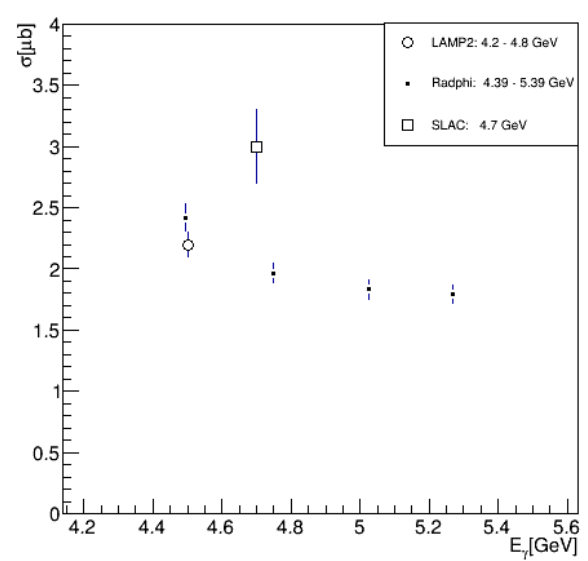

(a)

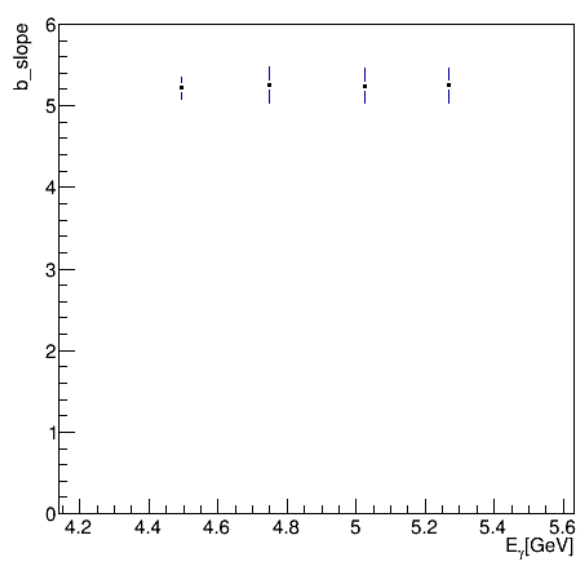

(b)

Figure 5.4.1: The total $\omega$ photoproduction cross section normalized per proton in the ${ }^{9} \mathrm{Be}$ target, cross section as a function of tagged photon energy(black dots, leftpanel). Measurements from previous experiments are shown for comparison. The right panel shows the slope of the -t distributions as a function of tagged photon energy.

The left panel in Figure 5.4.1 shows the measured total $\omega$ photoproduction cross section normalized per proton in the ${ }^{9} \mathrm{Be}$ target, as a function of the tagged photon energy. A $500 \mathrm{MeV}$ cut on the difference between the total shower and tagger energy has been applied in addition to the cuts described in sections 2 and 3. Additionally sideband subtraction around the diffractive $\Delta \phi$ peak has been applied in order to suppress contributions from reactions with $\mathrm{N}^{*}$ resonances in the final state. The open symbols are the results for $\gamma p \rightarrow \omega p$ from earlier 
measurements from LAMP2 [lamp2] and SLAC [4].

To investigate the dependence of the differential cross section on incident photon energy, the differential cross section $\frac{d \sigma}{d t}$ has been fitted to an exponential form $A e^{b t}$ for each photon energy. Side-band subtraction has been applied to the $\omega$ peaks in $3 \gamma$ invariant mass peaks to produce experimental t distributions for diffractive $\omega$ photoproduction. The fit results for the slope parameter $b$ as a function of incident photon energy are shown in Figure 5.4.1 right panel. The slope parameter is almost independent of energy within the displayed errors. These errors are dominated by the systematic error associated with the choice of fitting range in -t, $0.1-0.6 \mathrm{GeV}^{2}$ for the presented results, which leads to the strong correlation seen between the points in this plot.

Figure 5.4.2 shows the differential cross section for omega photoproduction as a function of $|t|$. To form this plot, data from all tagged photon energies have been summed. The vertical error bars reflect statistical errors, while the horizontal errors indicate the t-resolution within that t-bin. Data from previous experiments [Lamp2],[4] are shown as open symbols. Overall agreement between the different data sets is observed. At very low $|\mathrm{t}|<0.2 \mathrm{GeV}^{2}$ the Radphi data shows a depletion in the differential cross section compared to experiments performed on a hydrogen target. Such an effect is expected because of nuclear final state interactions. The exponential slope parameter $\mathrm{b}=4.6 \pm 0.4 \mathrm{GeV}^{-2}$ is obtained by fitting the acceptance corrected differential cross section within the range 0.1 $<|\mathrm{t}|<0.6 \mathrm{GeV}^{2}$. This value is smaller than the previous result $\mathrm{b}=7.1 \pm 0.9$ 


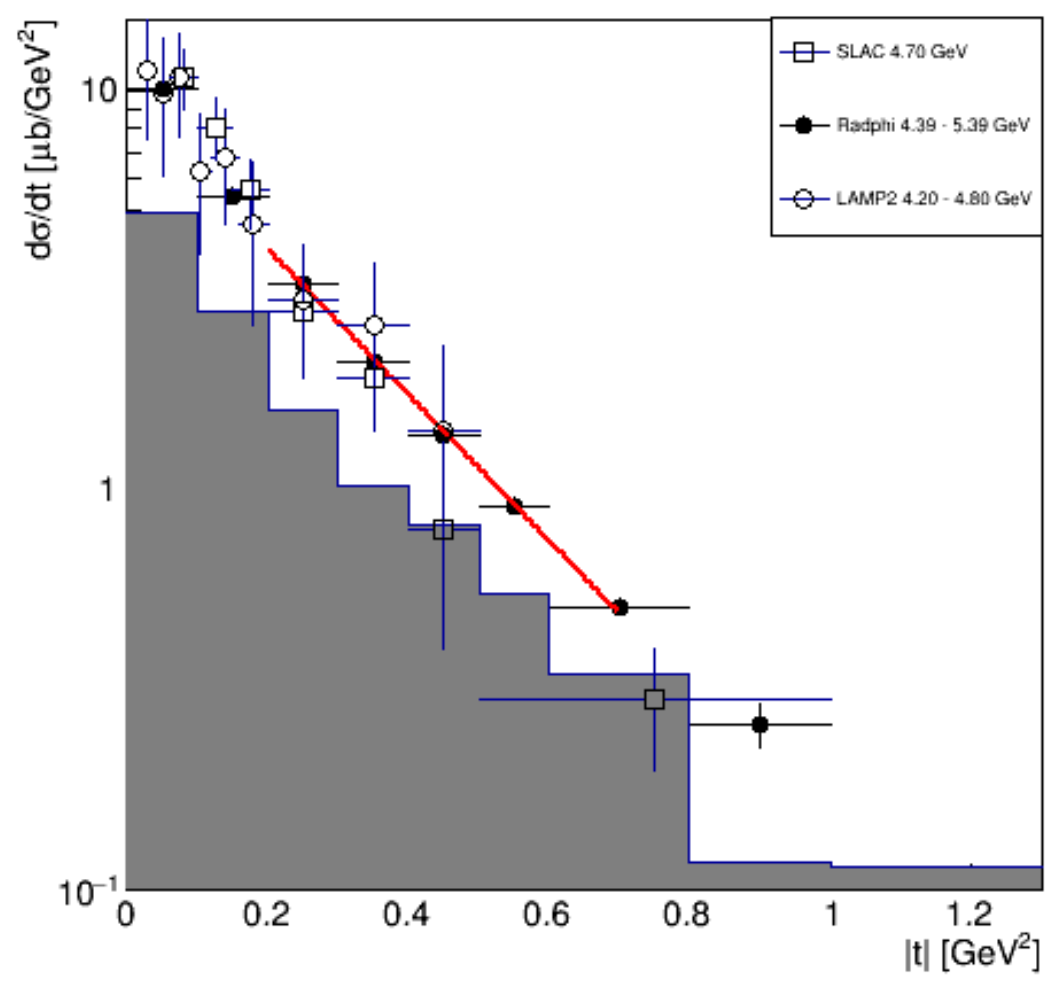

Figure 5.4.2: Differential cross section for $\gamma p \rightarrow \omega p$ as a function of $|\mathrm{t}|$. To form this plot data from all tagged photon energies shown in Figure 5.4.1 have been summed. Vertical error bars represent statistical error while the horizontal error bars reflect the bin size. The systematic error is shown as the grey shaded area at the bottom of the plot. 
obtained from the range $0.06<|\mathrm{t}|<0.5 \mathrm{GeV}^{2}$ at a photon energy of $4.7 \mathrm{GeV}^{2}$ [4]. However it is compatible with CLAS measurement of $5.4 \pm 0.6 \mathrm{GeV}^{-2}$ at 3.6 $\mathrm{GeV}[8]$ given that b does not appear to vary rapidly with incident energy.

\subsection{Unpolarized Spin Density Matrix Elements}

The unpolarized spin density matrix elements for $\omega$ photoproduction in the helicity and GJ frame for $\omega$ radiative decay are shown in Figure 5.5.1 and Figure 5.5.2, respectively.

In both frames, non-zero values for the diagonal element $\rho_{00}^{0}$ are observed. The results show a strong dependence of the spin density matrix elements with momentum transfer $|\mathrm{t}|$. In the helicity frame, non zero values for the diagonal element $\rho_{00}^{0}$ are also observed. The finite values of $\rho_{00}^{0}$ at low $|\mathrm{t}|$ indicates presence of amplitudes violating s-channel helicity. The off-diagonal elements $\operatorname{Re} \rho_{10}^{0}$ and $\rho_{1-1}^{0}$ are non zero and small at all $|\mathrm{t}|$ implying that there exists a weak interference between helicity flip and non-flip amplitudes. Since the values of these off-diagonal elements are small, this implies that small helicity flip contributions for are observed.

In the Gottfried Jackson system, the $\rho_{00}^{0}$ values are large implying a strong violation of helicity conservation in the t-channel. Increasing violation of t-channel helicity conservation is observed as $|\mathrm{t}|$ increases. At $|\mathrm{t}|>0.6 \mathrm{GeV}^{2}$ this trend seems to reverse and the $\rho_{00}^{0}$ values start decreasing as $|\mathrm{t}|$ increases. Small non zero values for the off diagonal elements $\operatorname{Re} \rho_{10}^{0}$ are observed at all $|\mathrm{t}|$ intervals. 


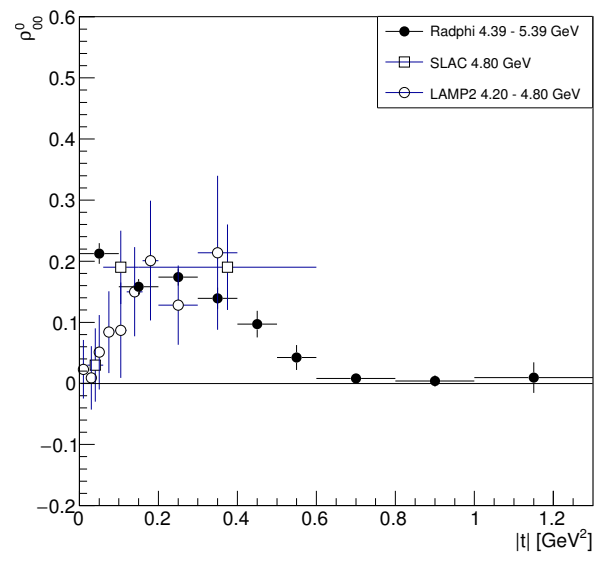

(a)

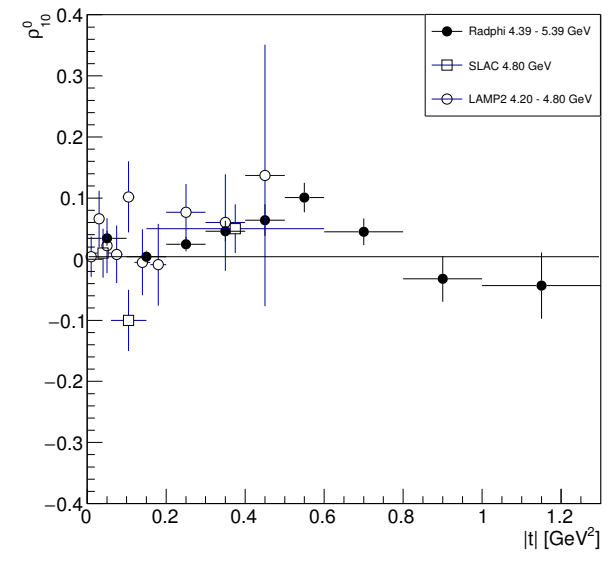

(b)

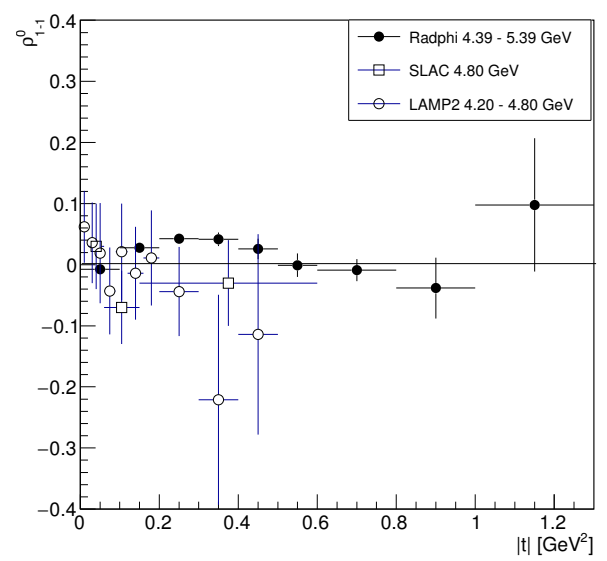

(c)

Figure 5.5.1: Spin density matrix elements for the reaction $\gamma p \rightarrow \omega p$ on a nuclear target in the helicity frame, as a function of momentum transfer $|\mathrm{t}|$. The vertical error bars indicated are statistical. SDMEs published by previous experiments are represented by open squares SLAC [4] and open circles LAMP2 collaboration [5]. 


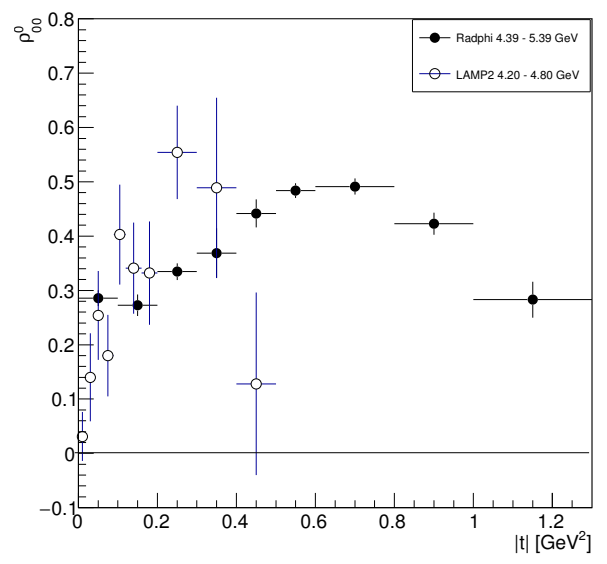

(a)

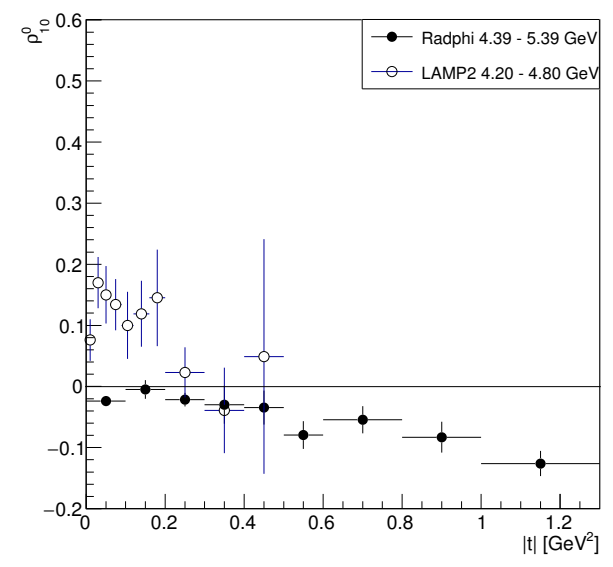

(b)

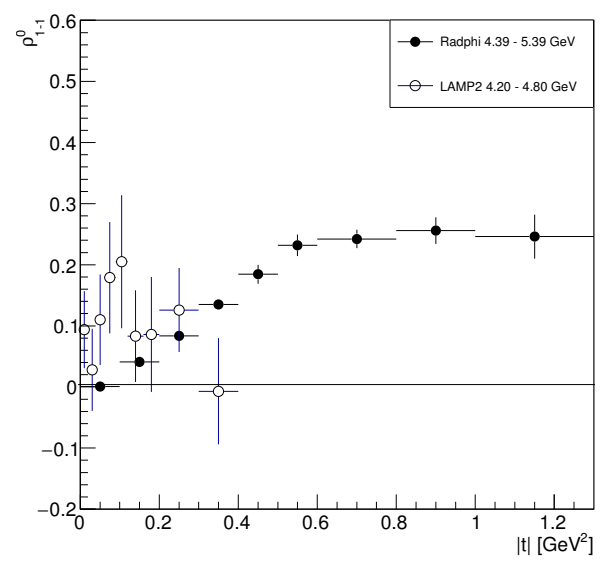

(c)

Figure 5.5.2: Gottfried Jackson spin density matrix elements for the reaction $\gamma p \rightarrow \omega p$ on a nuclear target, as a function of $|\mathrm{t}|$ in the energy range $4.39-5.39$ $\mathrm{GeV}$. The vertical error bars are statistical. Results published by the LAMP2 collaboration are represented by open circles [5]. 
However, finite non zero values for the double flip term $\rho_{1-1}^{0}$ are observed.

\section{Energy Dependance of SDMEs}

Figure 5.5.3 -Figure 5.5.8 shows the energy dependance of the SDME in both helicity and Gottfried Jackson frames. The angular distributions are binned in 4 energy bins with variable bin sizes, (4.39-4.60,4.60-4.90,4.90-5.15,5.15-5.39)GeV and 3 t-bins with the t-bin widths described below. To study this energy dependance,the angular distribution from each energy bin were binned in 3 t-bin regions and fitted to extract the SDMEs. Since binning in energy would lead to a reduction in statistics, the t-bin regions described below were selected to account for this reduction in statistics. Although the t-bins are wider than the ones used when doing the integrated energy analysis. The results obtained are consistent with Figure 5.5.1-Figure 5.5.2 and they show the same trend.

(i) low $|\mathrm{t}|$ region chosen in the $|\mathrm{t}|$ range $\left(\begin{array}{lll}0.0 & 0.3) \mathrm{GeV}^{2}\end{array}\right.$

(ii) mid $|\mathrm{t}|$ region chosen in the $|\mathrm{t}|$ range $(0.30 .6) \mathrm{GeV}^{2}$

(iii) high $|\mathrm{t}|$ region chosen in the $|\mathrm{t}|$ range $(0.61 .3) \mathrm{GeV}^{2}$

In the helicty frame,the SDMEs are independent of energy for each t-bin Each energy bin shows the same behavior, violation of helicity in the s-channel with the magnitude of violation decreasing as $|\mathrm{t}|$ increases. Likewise in the Gottfried Jackson frame,the SDMEs show the same behavior. The SDMEs are independent of energy for the selected t-bins. They reveal an increasing violation of t-channel helcity as $|\mathrm{t}|$ increases. 

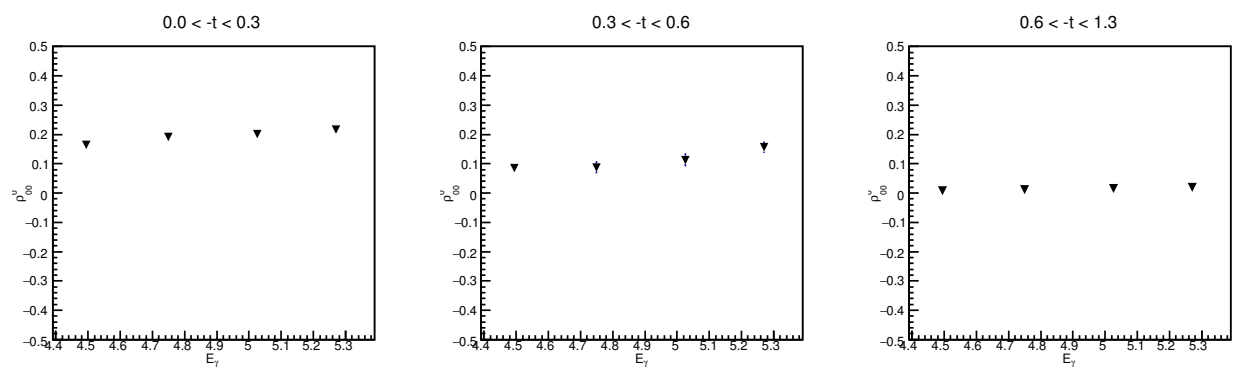

Figure 5.5.3: SDME component $\rho_{00}^{0}$ for different incident photon energies and selected t-bin ranges in the Helicity frame.
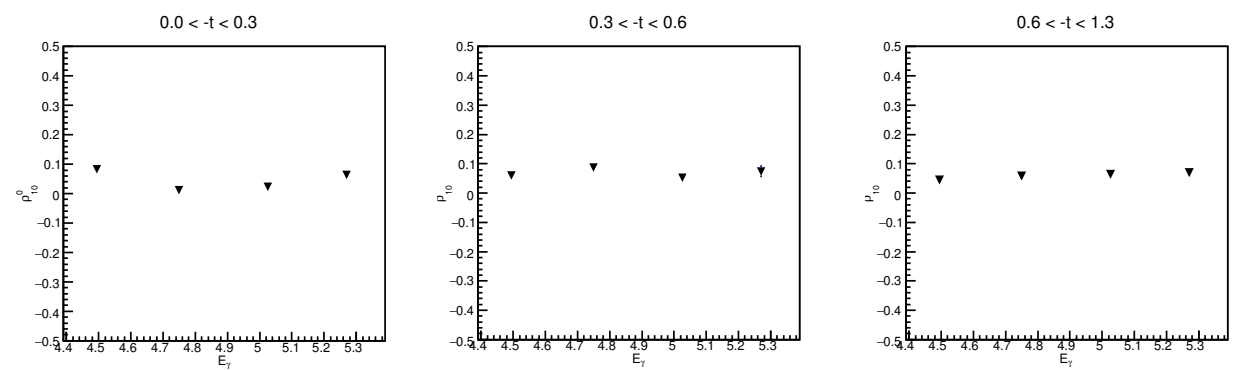

Figure 5.5.4: SDME component $\operatorname{Re} \rho_{10}^{0}$ for different incident photon energies and selected t-bin ranges in the Helicity frame.
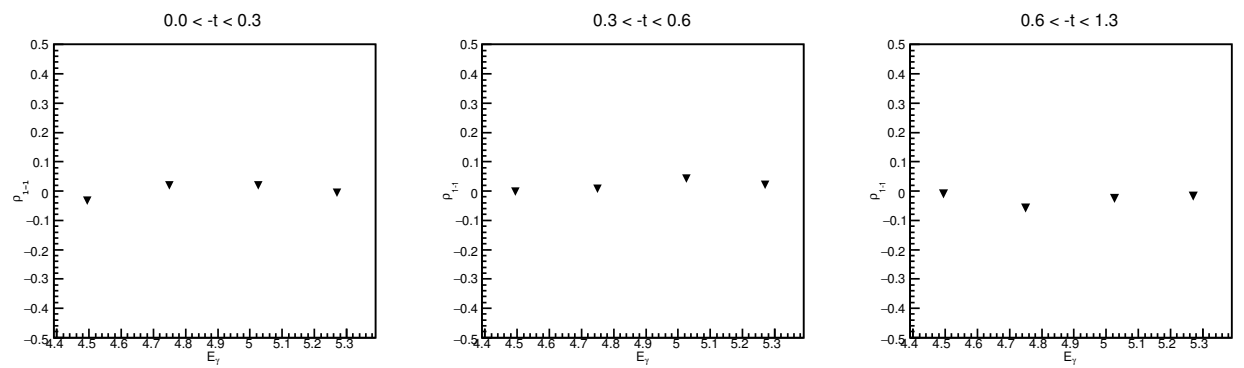

Figure 5.5.5: SDME component $\rho_{1-1}^{0}$ for different incident photon energies and selected t-bin ranges in the Helicity frame.
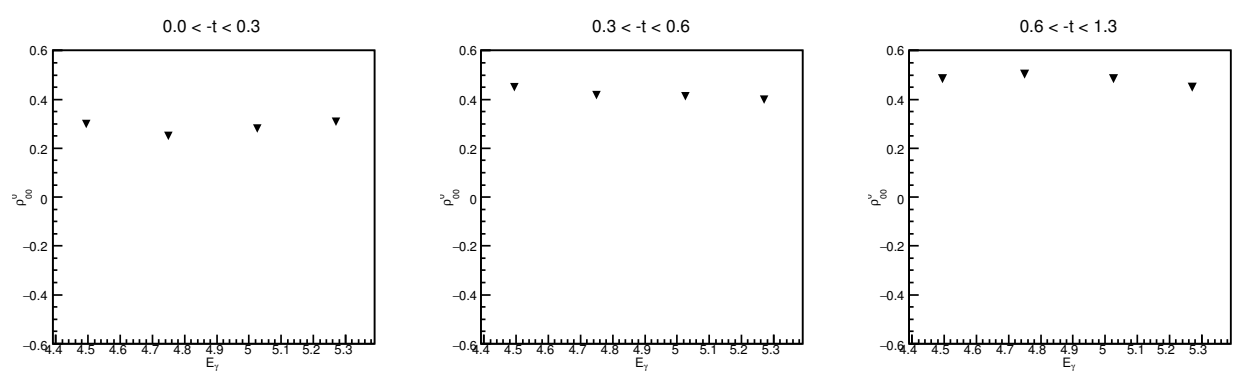

Figure 5.5.6: Incident energy dependance of $\rho_{00}^{0}$ for selected t-bins in the Gottfried Jackson frame. 

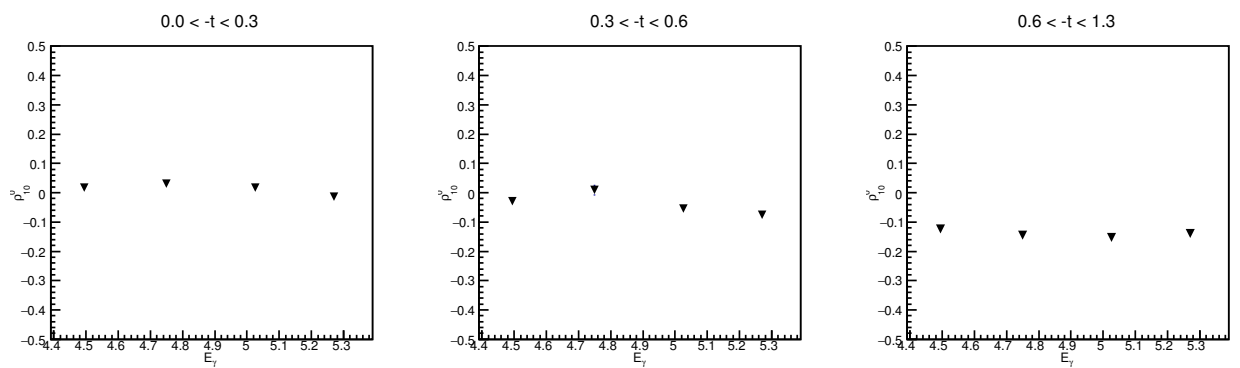

Figure 5.5.7: Incident energy dependance of the $\operatorname{Re} \rho_{10}^{0}$ for selected t-bins in the Gottfried Jackson frame.
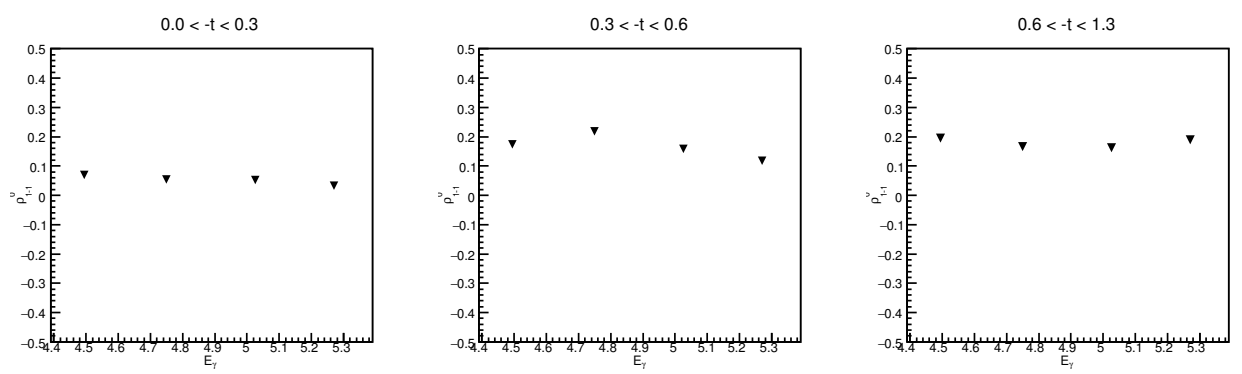

Figure 5.5.8: Incident beam dependanceof the $\rho_{1-1}^{0}$ component for selected t-bins in the Gottfried Jackson frame.

\subsubsection{Comparison with earlier results}

Lamp2 [5] publish results for all the three unpolarized SDMEs in both Gottfried Jackson and Helicity frame, total cross section and differential cross section in the energy range (4.2-4.8) $\mathrm{GeV}$ and $-\mathrm{t}=0.0-0.5 \mathrm{GeV}^{2}$. These results are compared to our results as shown in Figure 5.5.1 - Figure 5.5.2. There apears to be a good agreement between the two results for $|\mathrm{t}|>0.1 \mathrm{GeV}^{2}$.

SLAC [4] publish results for all SDMEs in the Helicity frame and differntial cross section obtained using a polarized photon beam with energy $4.7 \mathrm{GeV}$. We compare the unpolarized components to our results for $-\mathrm{t}=(0.0-0.6) \mathrm{GeV}^{2}$ and the agreement is good for $|\mathrm{t}|>0.1 \mathrm{GeV}^{2}$ 


\subsection{Theoretical Model for Vector Meson Photoproduction}

The extraction of the differential cross section and SDMEs has been discusssed in detial in the previous sections. After extracting these observables, we can use them to understand the $\gamma p \rightarrow \omega p$ production mechanism. And in our case since Radphi utilized a nuclear target, we can study what effect this has on the production mechanism.

\subsubsection{The Joint Physics Analysis Center (JPAC) Model}

The model developed by JPAC [34] incorporates a psudoscalar mesons $\pi^{0}$ and $\eta$ for the unnatural exchanges and the Pomeron, $\mathrm{a}_{2}$ and $\mathrm{f}_{2}$ natural exchanges in the t-channel.The schematic representation of this model is shown Figure 5.6.1. The

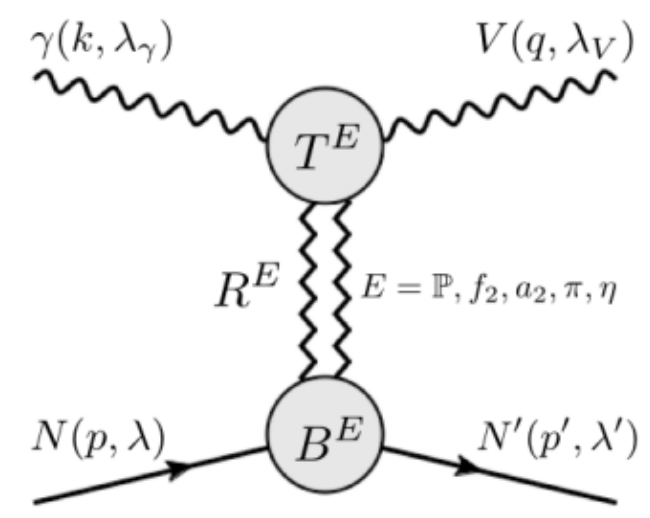

Figure 5.6.1: Schematic representation of t-channel meson and Pomeron exchanges [34]. 
helicity amplitude model is written as

$$
M_{\lambda_{V}, \lambda_{\gamma}, \lambda^{\prime}, \lambda}(s, t)=\sum_{E=\pi, \eta, \mathbb{P}, f_{2}, a_{2}} M_{\lambda_{V}, \lambda_{\gamma}, \lambda^{\prime}, \lambda}^{E}(s, t)
$$

The helicities are defined in the center of mass frame. The $\rho_{00}^{0}$ term in the helicity frame, decreases as $|\mathrm{t}|$ increases in the data while in the model it increases as $|\mathrm{t}|$ increases. The agreement between the model and the off-diagonal elements is somewhat good for the lower to mid $|t|$ ranges. For $|t|>0.6$ our measuremnts (off-diagonal) deviate from the model. The parmeters used for this model are determind using the SLAC data ??.

In the GJ frame,Figure 5.6.3, the model predicts the $\rho_{00}^{0}$ term increases as $|\mathrm{t}|$ increases for $|\mathrm{t}|<0.35$ in this region the model seems to reproduce our measurements. For $|\mathrm{t}|>0.35 \mathrm{GeV}^{2}$ the model has a turning point and the trend reverses which is contradicting to what we see in our measurements. Our measurents show a turing point but at a higher $|\mathrm{t}|$ value. Similarly the off-diagonal elements are not reproduced by the model. 


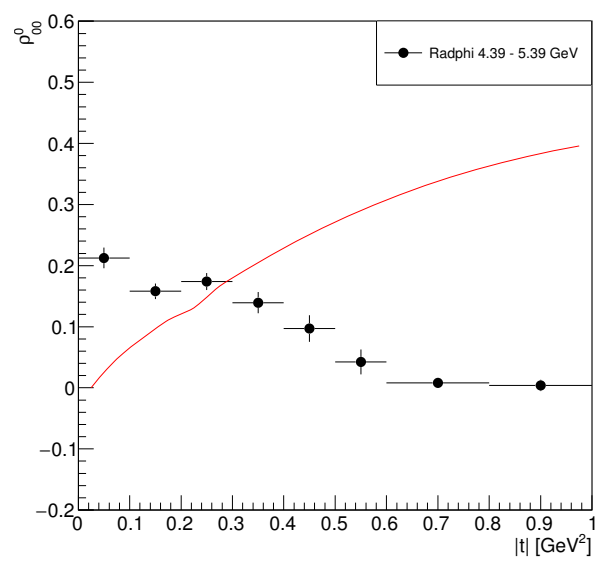

(a)

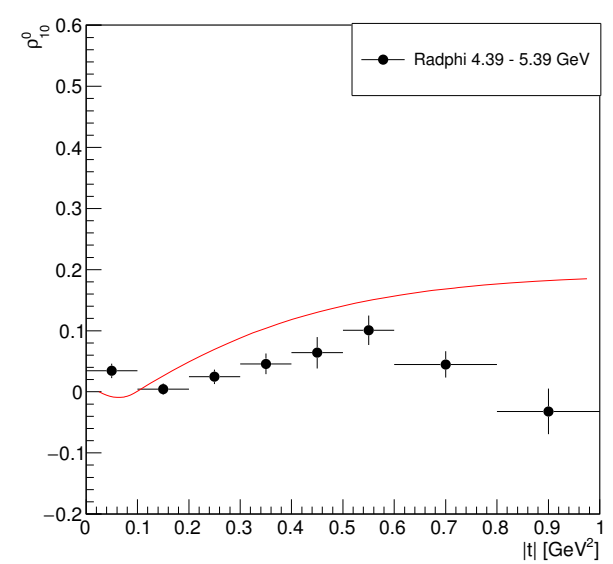

(b)

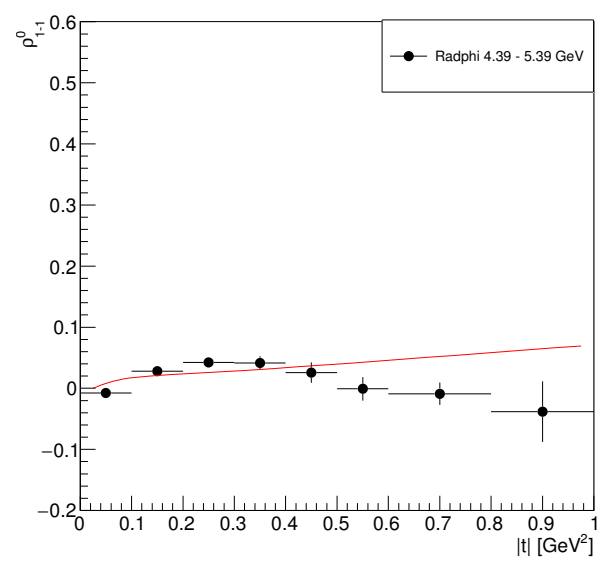

(c)

Figure 5.6.2: Helicity frame comparison of the Radphi experiment SDMEs to the JPAC model. 


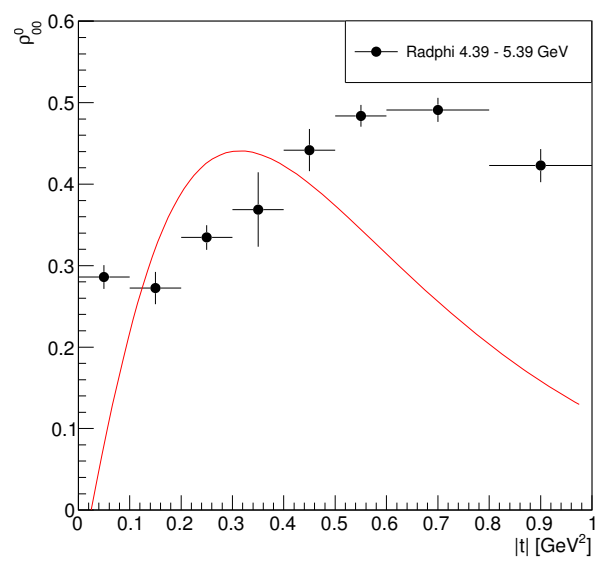

(a)

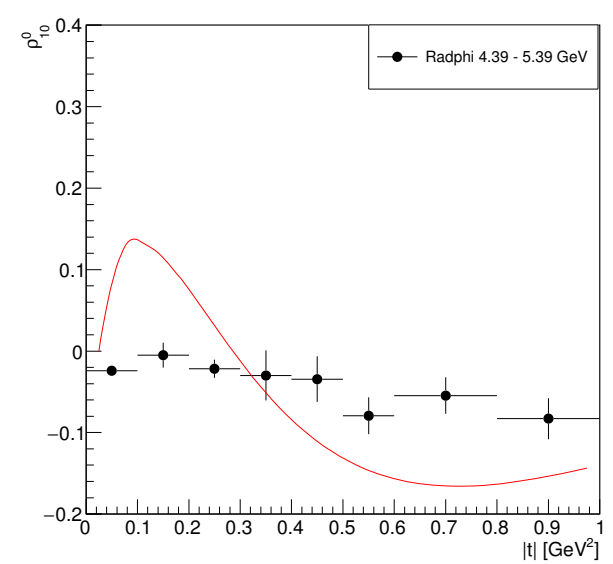

(b)

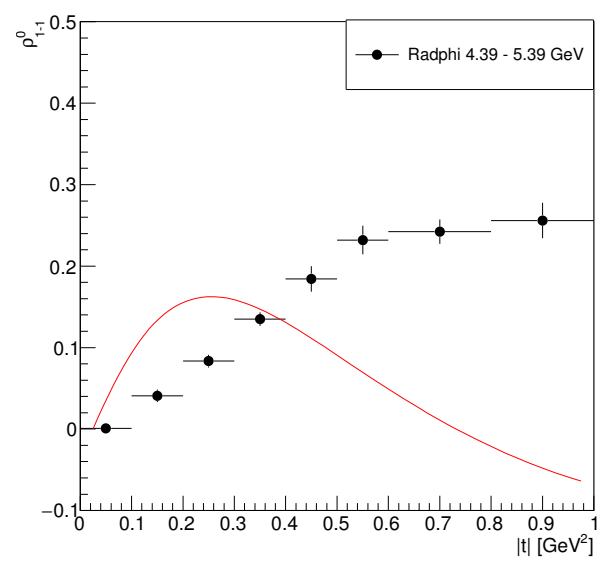

(c)

Figure 5.6.3: The JPAC model compared to our measurements in the Gottfried Jackson frame. 


\section{Chapter 6}

\section{Conclusions}

The purpose of the Radphi experiment was to investigate the radiative decays of vector mesons as a probe of meson structure. The radiative decay $\omega \rightarrow \pi^{0} \gamma$ has a much larger branching ratio of $8.28 \%$ compared with the $10^{-4}$ branching ratios of the $\phi$ to the light scalars, and so provides a starting point for that investigation. Data from the Radphi experiment have been analyzed to examine $\omega$ production from the reaction $\gamma p \rightarrow \omega p$ observed in the decay mode $\omega \rightarrow \pi^{0} \gamma$. A number of offline cuts have been devised, together with the tagging analysis, to isolate a relatively clean $\omega$ signal seen in the $3 \gamma$ invariant mass distribution. The $\omega$ yield extracted from the invariant mass distribution has been used to extract a total $\omega$ photoproduction cross section on a beryllium target. That cross section can be converted to a $\gamma p \rightarrow \omega p$ cross section by dividing by the atomic number of beryllium, under the assumption that nuclear medium modification effects are negligible for the kinematics selected by the recoil proton trigger, with the possible exception of $|\mathrm{t}|<0.1 \mathrm{GeV}^{2}$ where some indication of nuclear suppression relative to the free nucleon cross section is observed.

In order to compare photoproduction from bound protons with that from free protons, it was necessary to account for the momentum distribution of the bound 
protons in the initial state. For this purpose, a simple spectator model was developed that employed three free parameters to describe the proton in the nuclear medium. Using this model, MC simulations were able to reproduce the distribution of the missing momentum carried away by the unobserved recoil A $=8$ system.

The Radphi measurement of the $\omega$ photoproduction cross section after subtraction of non-diffractive contributions, agrees well with previous measurements of $\gamma p \rightarrow$ $\omega p$. The total cross section is almost constant with increasing beam energy as expected. The shape of the differential cross section $\frac{d \sigma}{d t}$ as a function of $|\mathrm{t}|<$ $1.0 \mathrm{GeV}^{2}$ is in agreement with previous measurements. It shows an exponential drop off for $|\mathrm{t}|<1.0 \mathrm{GeV}^{2}$. The average slope is smaller than previously reported at the Radphi energy, but it is consistent with previous measurements at lower energies.

The unpolarized SDMEs were extracted from the same data by fitting angular distributions in the helicity and Gottfried Jackson frames. The 3 independent unpolarized SDMEs were extracted in 9 t-bins which allowed the t-dependence of the SDMEs to be investigated. There appears to be a t-dependence of the SDMEs particularly in the helicity frame, where the $\rho_{00}^{0}$ values seem to decrease with increasing $|\mathrm{t}|$.

Under the hypotheses of s-channel and t-channel helicity conservation, all the matrix elements except the $\rho_{11}^{0}$ are expected to be zero in their respective frames. In the Gottfried Jackson frame, conservation of helicity indicates the dominance 
of $0^{+}$exchange in the t-channel. However, in the helicity frame, conservation of helicity does not follow from any simple s-channel process, but emerges as an empirical consensus of prior measurements in vector photoproduction at intermediate energies known as s-channel helicity conservation. The measured SDMEs reported in this paper show non-zero amplitudes for helicity flips between the incoming beam and outgoing vector meson in both s-channel and t-channel frames, revealing a violation of both s-channel and t-channel helicity conservation. Even though these results contradict the previous consensus regarding s-channel helicity conservation in vector photoproduction at these energies, in fact their agreement with previous SDME measurements [4][Lamp2] is actually quite good. The authors are not aware of any prior measurements of exclusive omega photoproduction from a nuclear target at these energies in which polarization observables have been analyzed. Plans are being formed to carry out a systematic study of the A-dependence of polarized vector photoproduction from nuclear targets in Hall D at Jefferson Lab [12]. This A-dependence is sensitive to models of how the $\omega-\mathrm{N}$ scattering cross section depends on polarization of the $\omega$ meson. The results presented in this thesiss represent a first step toward the goals of that program. 


\section{Chapter 7}

[1] MN Achasov, SE Baru, KI Beloborodov, AV Berdyugin, AV Bozhenok, AD Bukin, DA Bukin, SV Burdin, TV Dimova, SI Dolinsky, et al. Radiative decays of light vector mesons. Nuclear Physics A, 675(1-2):213-216, 2000 .

[2] Ugo Amaldi, M Jacob, and G Matthiae. Diffraction of hadronic waves. Annual Review of Nuclear Science, 26(1):385-456, 1976.

[3] D Amati. Me rose-elementary theory of angular momentum. Il Nuovo Cimento (1955-1965), 16(6):1160-1160, 1960.

[4] Joseph Ballam, GB Chadwick, Y Eisenberg, E Kogan, KC Moffeit, P Seyboth, IO Skillicorn, H Spitzer, G Wolf, HH Bingham, et al. Vector-meson production by polarized photons at 2.8, 4.7, and 9.3 gev. Physical Review D, $7(11): 3150,1973$.

[5] DP Barber, JB Dainton, LCY Lee, R Marshall, JC Thompson, DT Williams, TJ Brodbeck, G Frost, GN Patrick, GF Pearce, et al. A study of the reactions $\gamma \mathrm{p} \omega \mathrm{p}$ and $\gamma \mathrm{p} \omega \mathrm{p}$ and $\gamma \mathrm{p} \omega \delta+$. Zeitschrift für Physik C Particles and Fields, 26(3):343-351, 1984.

[6] IS Barker, E Gabathuler, and JK Storrow. wphotoproduction off neutrons: A test of a2 peripherality. Nuclear Physics B, 78(3):515-524, 1974.

[7] Vincenzo Barone and Enrico Predazzi. High-energy particle diffraction. Springer Science \&amp; Business Media, 2013.

[8] M Battaglieri, M Brunoldi, R De Vita, JM Laget, M Osipenko, M Ripani, M Taiuti, G Adams, MJ Amaryan, E Anciant, et al. Photoproduction of the $\omega$ meson on the proton at large momentum transfer. Physical review letters, 90(2):022002, 2003.

[9] T_H Bauer, RD Spital, DR Yennie, and FM Pipkin. The hadronic properties of the photon in high-energy interactions. Reviews of Modern Physics, $50(2): 261,1978$. 
[10] Claude Bernard, Tom Burch, Kostas Orginos, Doug Toussaint, Thomas A DeGrand, Carleton DeTar, Saumen Datta, Steven Gottlieb, Urs M Heller, and Robert Sugar. Qcd spectrum with three quark flavors. Physical Review D, 64(5):054506, 2001.

[11] Geoffrey F Chew and Steven C Frautschi. Regge trajectories and the principle of maximum strength for strong interactions. Physical Review Letters, 8(1):41, 1962.

[12] E Chudakov, Sergey Gevorkyan, and Alexander Somov. Photoproduction of $\omega$ mesons off nuclei and impact of polarization on the meson-nucleon interaction. Physical Review C, 93(1):015203, 2016.

[13] Suh Urk Chung. Spin formalisms. Technical report, Brookhaven Nat. Lab., 2008.

[14] Francis Edwin Close, A Donnachie, and Yu S Kalashnikova. Radiative decays of excited vector mesons. Physical Review D, 65(9):092003, 2002.

[15] Aachen Berlin Bonn Hamburg Heidelberg München Collaboration et al. Photoproduction of meson and baryon resonances at energies up to 5.8 gev. Physical Review, 175:1669-1696, 1968.

[16] Peter Donald Bruce Collins. An introduction to regge theory and high energy physics. An Introduction to Regge Theory and High Energy Physics, by PDB Collins, Cambridge, UK: Cambridge University Press, 2009, 2009.

[17] Jamil Daboul. Regge-pole description of the photoproduction of vector meson. Nuclear Physics B, 7(5):651-673, 1968.

[18] S. I. Dolinsky et al. Radiative Decays of $\rho$ and $\omega$ Mesons. Z. Phys., C42:511, 1989.

[19] S. I. "Dolinsky and others". "Radiative Decays of $\rho$ and $\omega$ Mesons". " $Z$. Phys.", "C42":"511",," 1989".

[20] Alexander Donnachie and Peter V Landshoff. Elastic scattering and diffraction dissociation. Nuclear Physics B, 244(2):322-336, 1984.

[21] W Drechsler. Regge-pole contribution to wo-photoproduction. Physics Letters, 23(4):272-274, 1966.

[22] Joshua P Ellis. Tikz-feynman: Feynman diagrams with tikz. Computer Physics Communications, 210:103-123, 2017.

[23] Harald Fritzsch, Murray Gell-Mann, and Heinrich Leutwyler. Advantages of the color octet gluon picture. Physics Letters B, 47(4):365-368, 1973. 
[24] E Gotsman, PD Mannheim, and U Maor. Photoproduction of vector mesons in a regge-pole model. Physical Review, 186(5):1703, 1969.

[25] E Gotsman and U Maor. Kinematical singularities and zeros for the reaction $\gamma \mathrm{n}$ vn. Physical Review, 171(5):1495, 1968.

[26] VN Gribov. Possible asymptotic behavior of elastic scattering. LETTERS TO THE EDITOR, 479:1, 1961.

[27] David J Gross and Frank Wilczek. Ultraviolet behavior of non-abelian gauge theories. Physical Review Letters, 30(26):1343, 1973.

[28] M Jacob and Gr C Wick. On the general theory of collisions for particles with spin. Annals of Physics, 7(4):404-428, 1959.

[29] RJ Jaffe. Multiquark hadrons. i. phenomenology of q 2 q 2 mesons. Physical Review D, 15(1):267, 1977.

[30] RT Jones, T Bogue, BE Evans, M Kornicer, AR Dzierba, R Gardner, JL Gunter, D Krop, R Lindenbusch, DR Rust, et al. Performance of the radphi detector and trigger in a high rate tagged photon beam. Nuclear Instruments and Methods in Physics Research Section A: Accelerators, Spectrometers, Detectors and Associated Equipment, 570(3):384-398, 2007.

[31] Mihajlo Kornicer. The search for radiative decays of vector mesons in the radphi experiment. 2006.

[32] J-M Laget. Photoproduction of vector mesons at large momentum transfer. Physics Letters B, 489(3):313-318, 2000.

[33] David WGS Leith, Scottish Universities, A Part, and B Part. Photoproduction and Vector Dominance. Citeseer, 1970.

[34] V Mathieu, J Nys, C Fernández-Ramírez, A Jackura, A Pilloni, N Sherrill, A Szczepaniak, G Fox, JPAC Collaboration, et al. Vector meson photoproduction with a linearly polarized beam. arXiv preprint arXiv:1802.09403, 2018.

[35] Curtis A Meyer and Y Van Haarlem. Status of exotic-quantum-number mesons. Physical Review C, 82(2):025208, 2010.

[36] Yongseok Oh, Alexander I Titov, and T-SH Lee. Nucleon resonances in $\omega$ photoproduction. Physical Review C, 63(2):025201, 2001.

[37] M Pivk and FL Diberder. splot: A statistical tool to unfold data distributions, 275 nucl. instrum. meth. a555 (2005) 356. arXiv preprint physics/0402083, 276. 
[38] JJ Sakurai. Theory of strong interactions. Annals of Physics, 11(1):1-48, 1960.

[39] JJ Sakurai. Vector-meson dominance and high-energy electron-proton inelastic scattering. Physical Review Letters, 22(18):981, 1969.

[40] K Schilling, P Seyboth, and G Wolf. On the analysis of vector-meson production by polarized photons. Nuclear Physics B, 15(2):397-412, 1970.

[41] K Schilling and F Storim. Analysis of the photoproduction of $\omega$ mesons. Nuclear Physics B, 7(5):559-573, 1968.

[42] Kamal K Seth. Hybrids and glueballs: A status report. Modern Physics Letters A, 18(02n06):330-339, 2003.

[43] A Sibirtsev, Ch Elster, and J Speth. Coherent $\omega$ photoproduction from nuclei and $\omega$ properties in nuclear matter. arXiv preprint nucl-th/0203044, 2002.

[44] A Sibirtsev, Kazuo Tsushima, and S Krewald. Systematic regge theory analysis of $\omega$ photoproduction. Physical Review C, 67(5):055201, 2003.

[45] Daniel I Sober, Hall Crannell, Alberto Longhi, SK Matthews, James T O'Brien, Barry L Berman, WJ Briscoe, Philip L Cole, JP Connelly, WR Dodge, et al. The bremsstrahlung tagged photon beam in hall b at jlab. Nuclear Instruments and Methods in Physics Research Section A: Accelerators, Spectrometers, Detectors and Associated Equipment, 440(2):263-284, 2000 .

[46] Steven Weinberg. Non-abelian gauge theories of the strong interactions. Physical Review Letters, 31(7):494, 1973.

[47] John Weinstein and Nathan Isgur. qq qqsystem in a potential model. Physical Review D, 27(3):588, 1983.

[48] Byung-Geel $\mathrm{Yu}$ and Kook-Jin Kong. Features of $\omega$ photoproduction off nucleon at forward angles (i): Dominance of $\pi$ exchange with regge cuts and scaling of differential cross sections. arXiv preprint arXiv:1710.04511, 2017.

[49] Qiang Zhao, JS Al-Khalili, and PL Cole. Vector meson photoproduction studied in its radiative decay channel. Physical Review C, 71(5):054004, 2005 . 